Westfälische Wilhelms-Universität Münster

Wintersemester 2019/2020

Fachbereich 14 - Geowissenschaften

Institut für Geoinformatik

Bachelorarbeit zur Erlangung des Grades Bachelor of Science der Geoinformatik

\title{
Ein Metadatenlabel für das semantische Sensorweb
}

Vorgelegt von:

Anika Graupner

E-Mail: a_grau05@uni-muenster.de

Erstgutachter: Dipl.-Geoinf. Daniel Nüst

Zweitgutachter: Hon.-Prof. Dr. Albert Remke

Abgabetermin: $\quad$ 17.02.2020

Bearbeitungszeit: 3 Monate 


\section{Abstract}

\section{Deutsch}

Das World Wide Web entwickelt sich zu einem semantischen Web. Daten werden mit Hilfe von Ontologien semantisch annotiert, um sie maschinenlesbar bereitzustellen. Zu diesen Daten zählen auch Sensordaten. Da die Anzahl an online zur Verfügung gestellten Sensordaten steigt, fällt es dem Nutzer immer schwerer, geeignete Daten zu finden. Die neuen Sensorbeschreibungen in Form von Ontologien werden in der folgenden Arbeit dazu eingesetzt, dem Nutzer die Suche wieder zu vereinfachen. Die Erfassung von Informationen aus den neuen maschinenlesbaren Beschreibungen kann für den Menschen schwierig sein. Daher wird das von GeoViQua ursprünglich für GEOSS entwickelte GEO Label dazu eingesetzt, auch visuelle Zusammenfassungen der Verfügbarkeit von Informationen in Sensorbeschreibungen bereitzustellen. Das Label besteht aus Facetten, die Auskunft über das Vorhandensein von

Informationen wie Herstellerprofil, Benutzerfeedback und Qualitätsinformationen in Metadatendokumenten geben. In der vorliegenden Arbeit wird die 52 North Implementierung der Java GEO Label API um die Generierung von Labels basierend auf der Semantic Sensor Network Ontology (SSNO) und verbundenen Linked Data Ontologien erweitert. Zuvor werden aus diesen Ontologien Informationsquellen für die Facetten des Labels gesucht. Zudem wird die GEO Label API mit einem Serverless Cloud-Computing Anbieter zur Verfügung gestellt, wobei die Frage beantwortet wird, welche Cloud-Ressourcen für die Labelgenerierung notwendig sind.

\section{Englisch}

The World Wide Web is developing into a semantic web. Data are semantically annotated using ontologies to make them available in a machine-readable form. These data include also sensor data. As the amount of sensor data made available online increases, it becomes more difficult for users to find suitable data. The new sensor descriptions in form of ontologies will be used in the following work to simplify the search for users again. The acquisition of information from the new machine-readable descriptions may be difficult for humans. Therefore, the GEO Label, originally developed by GeoViQua for GEOSS, is used to provide visual summaries of the availability of information in sensor descriptions. The label consists of facets that provide information about the availability of information in metadata documents such as producer profile, user feedback and quality information. In this thesis the 52 North implementation of the Java GEO label API is extended by the generation of labels based on the Semantic Sensor Network Ontology (SSNO) and related Linked Data Ontologies. Prior to this, information sources for the facets of the label are searched for from these ontologies. In addition, the GEO Label API will be made available with a serverless cloud computing provider, whereby the question will be answered which cloud resources are necessary for label generation. 


\section{Inhaltsverzeichnis}

Abstract

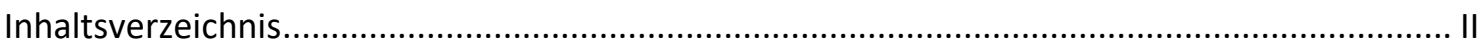

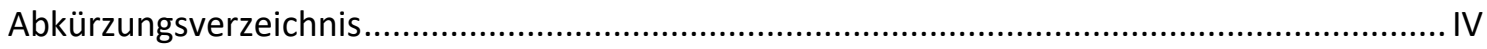

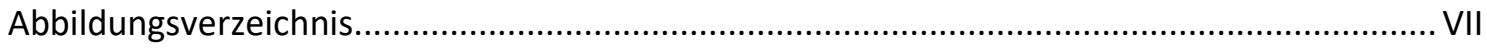

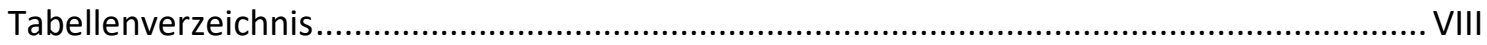

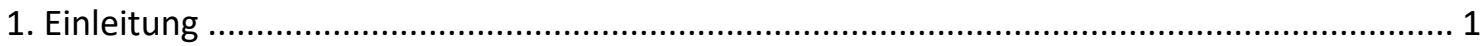

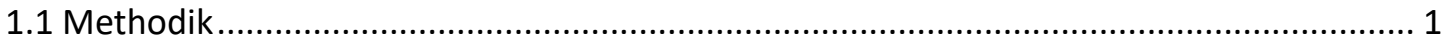

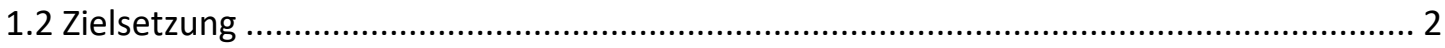

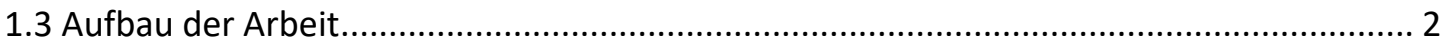

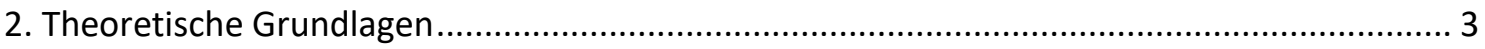

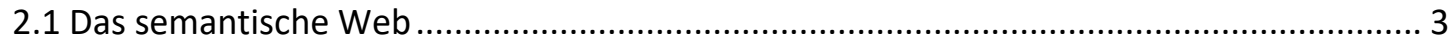

2.1.1 Linked (Open) Data ............................................................................................... 3

2.1.2 Standards im semantischen Web.......................................................................... 4

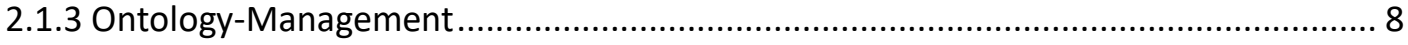

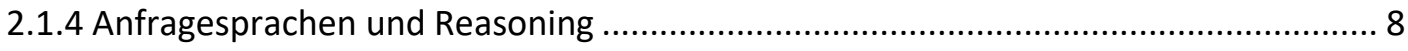

2.2 Webintegrierung von Sensoren und Sensordaten ........................................................ 9

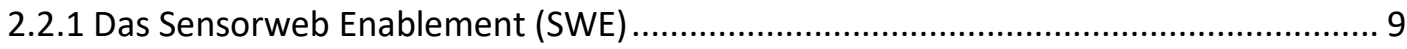

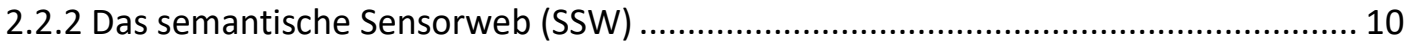

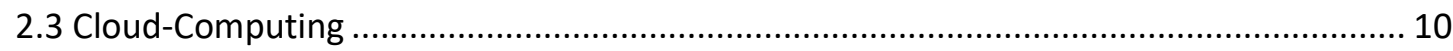

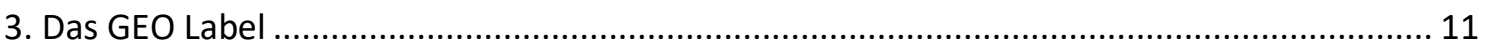

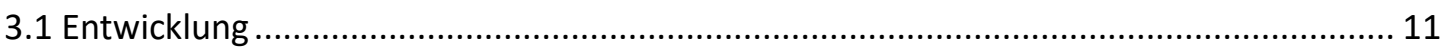

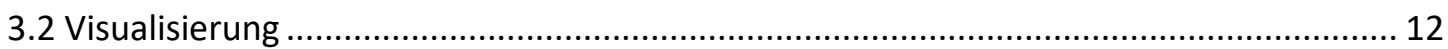

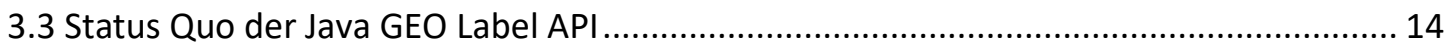

4. Evaluation der Semantic Sensor Network Ontology und der verbundenen Ontologien zur Wahl der Informationsquellen für die Facetten des GEO Labels............................................ 15

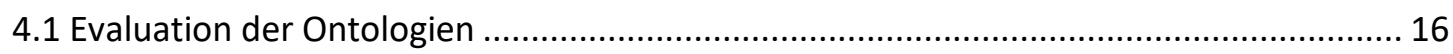

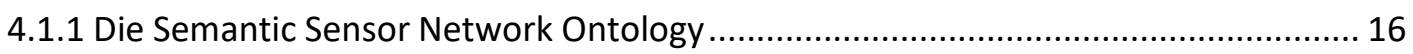

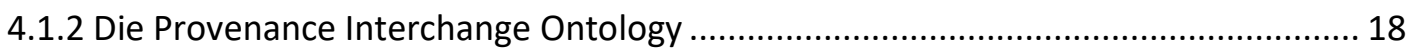

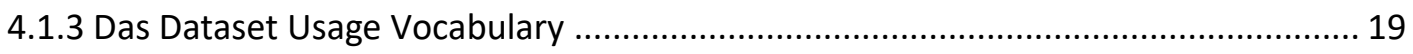

4.2 Wahl der Informationsquellen aus den evaluierten Ontologien für die Facetten des GEO

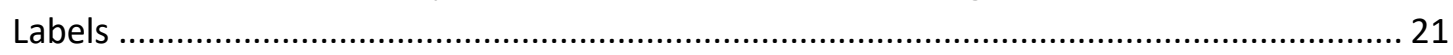

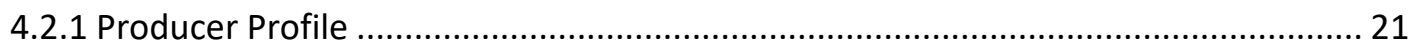

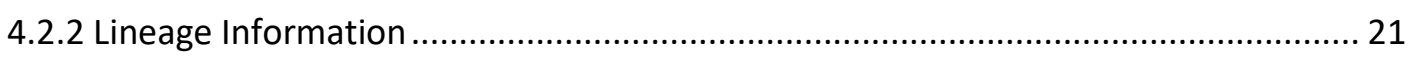

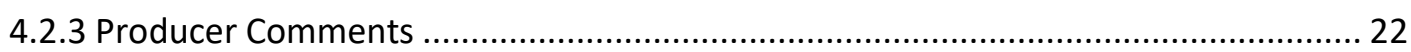




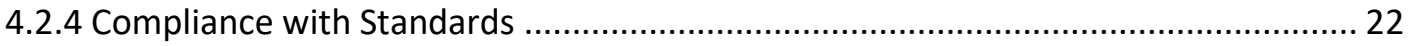

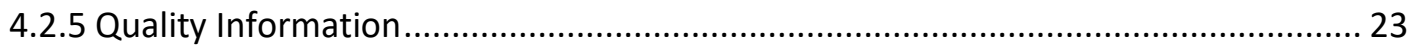

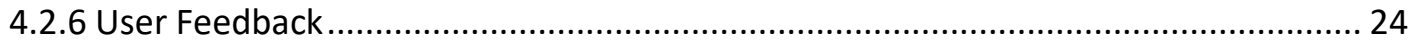

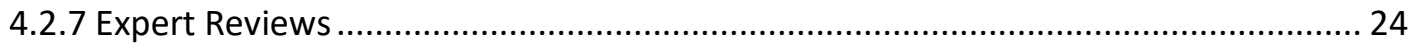

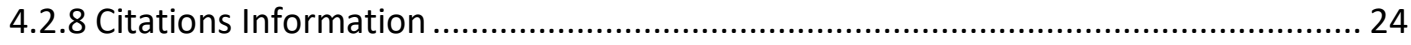

5. Vergleich von Anbietern für Serverless Cloud-Computing und Wahl eines geeigneten Anbieters für die Bereitstellung der Java GEO Label API ....................................................... 25

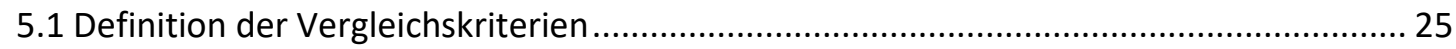

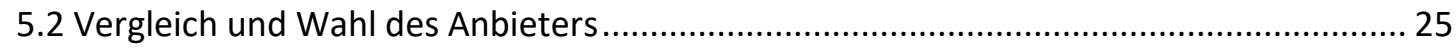

6. Erweiterung der Java GEO Label API um die prototypische Generierung SSNO-basierter Label und Bereitstellung als Cloud-basierte serverless Anwendung ................................................ 29

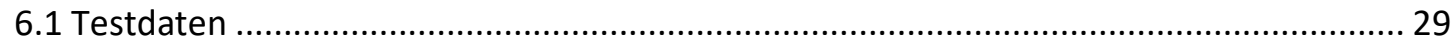

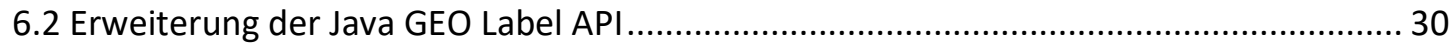

6.2.1 Transformationsdatei für SSNO-basierte Metadaten ............................................... 30

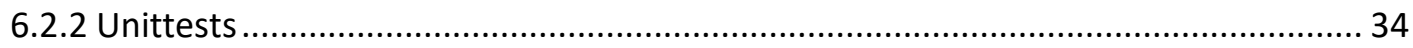

6.2.3 Java GEO Label API mit Docker Image lokal ausführen............................................ 34

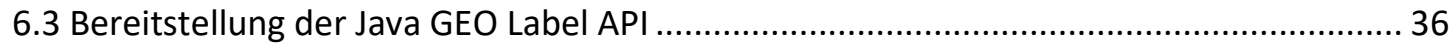

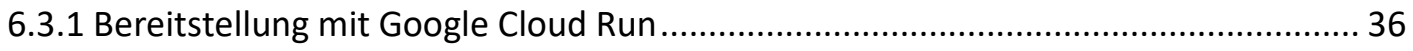

6.3.2 Bereitstellung mit AWS Lambda und Amazon API Gateway...................................... 38

6.3.3 Performanz-Tests mit Apache JMeter....................................................................... 40

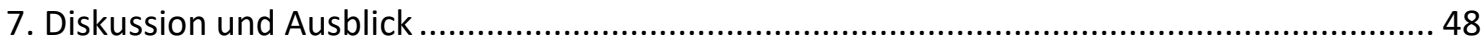

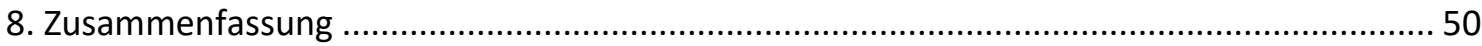

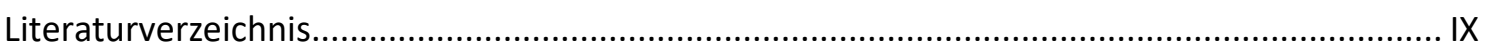

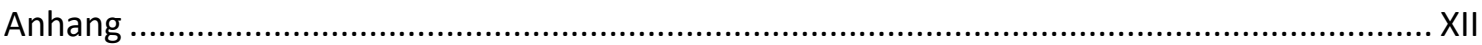

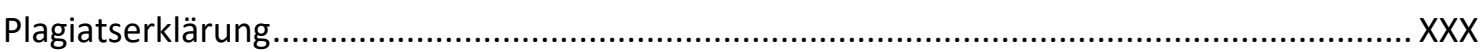




\section{Abkürzungsverzeichnis}

$52 \mathrm{~N}$

API

AWS

Bsp.

bspw.

bzw.

DCAT

DUV

ERC

FGDC

FOAF

FP7

GCP

GCR

GEO

GEOSS

GeoViQua

HTML

HTTP

IBM

IoT

ISO

JSON
52 North

Application Programming Interface

Amazon Web Services

Beispiel

beispielsweise

beziehungsweise

Data Catalog Vocabulary

Dataset Usage Vocabulary

Easy RDF Converter

Federal Geographic Data Committee

Friend of a Friend

7. Forschungsrahmenprogramm

Google Cloud Platform

Google Cloud Run

Group on Earth Observations

Global Earth Observation System of Systems

Quality aware Visualization for the Global Earth Observation System of systems

Hypertext Markup Language

Hypertext Transfer Protocol

International Business Machines Corporation

Internet of Things

International Organization for Standardization

Java Script Object Notation 


\begin{tabular}{|c|c|}
\hline $\mathrm{LH}$ & Local Host \\
\hline LML & Label Markup Language \\
\hline $\mathrm{MBC}$ & MyBluemix RDF Validator and Converter \\
\hline OGC & Open Geospatial Consortium \\
\hline o.J. & ohne Jahr \\
\hline o.V. & ohne Verfasser \\
\hline OWL & Web Ontology Language \\
\hline O\&M & Observation \& Measurement \\
\hline PNG & Portable Network Graphic \\
\hline PROV-O & Provenance Interchange Ontology \\
\hline RDF & Resource Description Framework \\
\hline RDFS & Resource Description Framework Schema \\
\hline REST & Representational State Transfer \\
\hline SDW & Spatial Data on the Web \\
\hline SensorML & Sensor Model Language \\
\hline SOS & Sensor Observation Service (SOS) \\
\hline SOSA & Sensor, Observation, Sample and Actuator \\
\hline SPARQL & SPARQL Protocol and RDF Query Language \\
\hline SSN & Semantic Sensor Network \\
\hline SSNO & Semantic Sensor Network Ontology \\
\hline SSN-XG & Semantic Sensor Network Incubator Group \\
\hline SSW & Semantic Sensor Web \\
\hline STC & Science and Technologie Committee \\
\hline SVG & Scalable Vector Graphic \\
\hline SWE & Sensor Web Enablement \\
\hline
\end{tabular}


URI

URL

vgl.

W3C

Web

XML

XPATH

z.B. zum Beispiel

Uniform Resource Identifier

Uniform Resource Locator

vergleich(e)

World Wide Web Consortium

World Wide Web

Extensible Markup Language

XML Path Language 


\section{Abbildungsverzeichnis}

Abbildung 1: Ein RDF-Graph mit Literalen zur Beschreibung von Datenwerten.

Abbildung 2: Visualisierung der Verfügbarkeitszustände der Facetten im GEO Label.

Abbildung 3: Beispiel für einen Hover-Over-Text für die Facette Producer Profile

Abbildung 4: Die SOSA und SSN Ontologien und ihre vertikalen und horizontalen Module..... 16

Abbildung 5: Übersicht über die SSN Klassen und Eigenschaften (Observation Perspektive)... 17

Abbildung 6: Beziehung zwischen SOSA und der PROV-Ontologie. 19

Abbildung 7: Überblick über den Aufbau des Dataset Usage Vocabulary. 20

Abbildung 8: Cloud Run-Ressourcenmodell.

Abbildung 9: GEO Label als Scalable Network Graphic, lokal generiert.

Abbildung 10: Google Cloud Run Dienst zur Bereitstellung der GEO Label API, aktuell mit einer Revision.

Abbildung 11: GEO Label als Portable Network Graphic, generiert über die mit Google Cloud Run bereitgestellte GEO Label API.

Abbildung 12: Mit Amazon API Gateway bereitgestellte API

Abbildung 13: GEO Label als Scalable Vector Graphic, generiert über die mit AWS Lambda und API Gateway bereitgestellte GEO Label API.

Abbildung 14: Tabellarisches Ergebnis allgemeine Tests Google Cloud Run API.

Abbildung 15: Tabellarisches Ergebnis allgemeine Tests AWS Lambda API.

Abbildung 16: Tabellarisches Ergebnis Szenario 1 Google Cloud Run API.

Abbildung 17: Tabellarisches Ergebnis Szenario 1 AWS Lambda API.

Abbildung 18: Tabellarisches Ergebnis Szenario 1 Local Host API.

Abbildung 19: Graphisches Ergebnis Szenario 2 Google Cloud Run API.

Abbildung 20: Graphisches Ergebnis Szenario 2 AWS Lambda API.

Abbildung 21: Graphisches Ergebnis Szenario 2 AWS Lambda API - Überarbeitete CloudRessourcen.

Abbildung 22: Graphisches Ergebnis Szenario 2 Local Host API. 


\section{Tabellenverzeichnis}

Tabelle 1: Die wichtigsten Klassen in RDF und RDFS. ........................................................... 7

Tabelle 2: Die wichtigsten vordefinierten Properties in RDF und RDFS.................................... 7

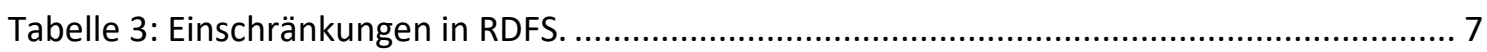

Tabelle 4: Symbole und Erläuterungen zu den einzelnen Facetten des GEO Labels................. 12

Tabelle 5: Präfixe und Namensräume der erwähnten Ontologien und Vokabulare. .................. 15

Tabelle 6: Vergleich von Anbietern für Serverless Cloud-Computing.......................................... 25 


\section{Einleitung}

Mit der wachsenden Bedeutung von Linked (Open) Data und der Weiterentwicklung des World Wide Web (kurz Web) zu einem semantischen Web, in dem Informationen mit eindeutigen Bedeutungen versehen werden, werden neue semantische Technologien entwickelt zur Erstellung, Verarbeitung und Analyse semantisch annotierter Daten. ${ }^{1}$

Auch das Sensorweb entwickelt sich durch die semantische Annotation von Sensordaten zu einem semantischen Sensorweb. ${ }^{2}$ Neben den gängigen Sensorweb-Standards wie der Sensor Model Language (SensorML) und dem Sensor Observation Service (SOS) werden neue Empfehlungen in Form von Ontologien für die Beschreibung von Sensordaten herausgegeben. Eine der bedeutendsten Ontologien ist hierbei die Semantic Sensor Network Ontology (SSNO). In dieser Arbeit soll diese Ontologie zusammen mit den verbundenen Linked Data Ontologien dazu verwendet werden, dem Nutzer von Sensordaten die Suche nach geeigneten Daten zu vereinfachen.

Da die Anzahl der online zur Verfügung gestellten Sensordaten insbesondere durch das Internet of Things (IoT) weiter steigt, wird es für den Nutzer immer schwieriger, die für ihn passenden Daten aus der Menge der Sensordaten zu filtern. ${ }^{3}$ Zudem basiert die Idee des semantischen Webs darauf, Daten maschinenlesbar und nicht direkt für den Menschen bereitzustellen. ${ }^{4}$ Die neuen Sensorbeschreibungen für das semantische Sensorweb sind somit für den Nutzer noch schwieriger zu verstehen.

\subsection{Methodik}

Um den Nutzer bei der Suche nach geeigneten Sensordaten zu unterstützen, soll das 2009 von GeoViQua ursprünglich für das Global Earth Observation System of Systems (GEOSS) entwickelte GEO Label verwendet werden. Dieses stellt eine visuelle Zusammenfassung der Verfügbarkeit von Metadaten für einen Datensatz dar. ${ }^{5}$

Dabei soll die Implementierung der GEO Label API von 52 North $(52 \mathrm{~N})$, die bereits die Generierung von Labels basierend auf Sensorbeschreibungen in SensorML und SOS ermöglicht, um die Label-Generierung aus SSNO-basierten Metadatendokumenten erweitert werden.

Da sich das Label aus verschiedenen Facetten zusammensetzt, die Auskunft über das Vorhandensein von Informationen in Metadatendokumenten wie zum Beispiel Herstellerprofil, Benutzerfeedback und Expertenbewertungen geben, müssen zunächst Informationsquellen in SSNO und den verknüpften Linkes Data Ontologien für diese Facetten gesucht werden. Die Evaluation der Ontologien erfolgt hauptsächlich durch Online-Recherche.

\footnotetext{
${ }^{1}$ Vgl. Gradmann, S. / Hennicke, S. / Olensky, M. (2012): Linked Data. In: Digitale Dienste für die Wissenschaft, Nr. 35, S. 18, http://doi.org/10.18452/6627, Zugriff am 31.12.2019.

${ }^{2} \mathrm{Vgl}$. Shet, A. / Henson, C. / Sahoo, S. (2008): Semantic Sensor Web. In: Internet Computing, IEEE, Nr. 12, S. 78, http://doi.org/10.1109/MIC.2008.87, Zugriff am 16.01.2020.

${ }^{3} \mathrm{Vgl}$. Nüst, D. \& Lush, V. (2015): A GEO label for the Sensor Web, https://doi.org/10.31223/osf.io/ka38z, Zugriff am 05.01.2020.

${ }^{4}$ Vgl. Hitzler, P. / Krötzsch, M. / Rudolph, S. / Sure, Y. (2008): Semantic Web, 1. Auflage, Berlin: Springer, S.1.

${ }^{5}$ Vgl. Nüst \& Lush (2015).
} 
Die anschließend erweiterte Implementierung der GEO Label API soll dem Nutzer als Cloudbasierte serverless Anwendung zur Verfügung gestellt werden. Dabei müssen zunächst verschiedene Cloud-Computing Anbieter anhand von zu definierenden Kriterien verglichen werden, um schließlich den am besten geeignetsten für die Bereitstellung der API auszuwählen. Zuletzt wird die Cloud-basierte Generierung mit einer Test-Suite getestet.

\subsection{Zielsetzung}

Ziel der Arbeit ist es, durch die Erweiterung der GEO Label API dem Nutzer von Sensordaten ein Label zur Verfügung zu stellen, das eine Übersicht über die Verfügbarkeit von Informationen in den Sensorbeschreibungen gibt, ohne dass der Nutzer sich diese aus den Metadatendokumente selbst erschließen muss. Auf dem Weg dorthin müssen mehrere Forschungsfragen beantwortet werden.

Zunächst muss entschieden werden, welche Informationsquellen in SSNO und verknüpften Linked Data Ontologien für die Facetten des GEO Labels geeignet sind und ob es Facetten gibt, für die überhaupt keine Informationsquellen vorhanden sind.

Weiterhin muss untersucht werden, in welchen Datenmodellen und Enkodierungen SSNObasierte Sensorbeschreibungen vorliegen und mit welcher Abfragesprache sich die Implementierung letztlich umsetzen lässt. Die Abfragesprache wird zur Suche nach den Informationen in einer Sensorbeschreibung benötigt.

Bei der Bereitstellung der GEO Label API als Cloud-basierte serverless Anwendung muss zusätzlich festgelegt werden, welche Cloud-Ressourcen für eine bedarfsorientierte Labelgenerierung notwendig sind und wie diese auf unterschiedliche Szenarien von Nutzeranfragen reagieren.

\subsection{Aufbau der Arbeit}

Anfänglich werden in Kapitel 2 die theoretischen Grundlagen erläutert, die zum Verständnis dieser Arbeit unumgänglich sind. Dazu zählen Inhalte zum semantischen Web und Sensorweb sowie elementare Informationen zum Cloud-Computing. Kapitel 3 gibt einen Überblick über das GEO Label, dessen Entwicklung und Visualisierung. Zudem wird der aktuelle Stand der JavaImplementierung der GEO Label API von 52 North erläutert. Die Evaluation der Semantic Sensor Network Ontology und verbundenen Ontologien folgt in Kapitel 4, zusammen mit der Erläuterung der Wahl der Informationsquellen. Anschließend werden in Kapitel 5 die Anbieter für serverless Cloud-Computing anhand von definierten Kriterien verglichen, damit sich für einen Anbieter entschieden werden kann. Die Umsetzung der Erweiterung der GEO Label API sowie die Bereitstellung über den zuvor gewählten Anbieter werden in Kapitel 6 beschrieben. In Kapitel 7 werden die Ergebnisse diskutiert, Kapitel 8 fasst die wichtigsten Aspekte der Arbeit noch einmal zusammen. 


\section{Theoretische Grundlagen}

Dieses Kapitel definiert zentrale Begriffe und erläutert die theoretischen Grundlagen, die zum Verständnis dieser Arbeit notwendig sind.

\subsection{Das semantische Web}

Das semantische Web (im Deutschen häufig auch Semantic Web genannt) ist eine Erweiterung des World Wide Web (kurz Web).

Die Idee für das Web entstand 1989 durch Tim Berners-Lee am Genfer Forschungsinstitut. Es ist ein Dienst des Internets, durch den es möglich ist, auf flexible Art und Weise Inhalte interaktiv und international bereitzustellen und auszutauschen. ${ }^{6}$ Hierbei ist der Mensch der Endnutzer, das heißt, dieser erfasst die Bedeutung einer Information auf einer Webseite selbst und kann diese auch zu anderen Informationen in Beziehung setzen. Die zunehmende Menge an zur Verfügung gestellten Inhalten und Daten im Web erschwert es dem Nutzer, die für ihn wichtigen Informationen zu erfassen. ${ }^{7}$ Deshalb besteht die Notwendigkeit, „(...) Daten maschinenlesbar und -interpretierbar bereitzustellen. ${ }^{18}$

„Ziel des Semantic Web ist es, neuartige Anwendungen und Dienste zu ermöglichen, indem Daten von Maschinen bzw. Software interpretiert und für vorher nicht notwendigerweise vorgesehene Zwecke wieder- und weiterverwendet werden. Um dies zu ermöglichen, werden Daten mit einer Struktur (Syntax) und Bedeutung (Semantik) versehen. ${ }^{\text {"g }}$

Die vorhandenen Webdokumente sollen um neue Daten und Metadaten in Form von Ontologien erweitert werden. „Der Begriff Ontologie ist (...) als äquivalent zum Begriff Wissensbasis zu verstehen und beschreibt ein (...) erstelltes Dokument, welches Wissen einer Anwendungsdomäne modelliert. ${ }^{\text {10 }}$

Hierbei spielt das World Wide Web Consortium (W3C) eine bedeutende Rolle. Dieses ist eine internationale Gemeinschaft, die Webstandards entwickelt, um das Web optimal nutzbar zu machen. ${ }^{11}$ Dabei werden auch Standards für das semantische Web erstellt.

\subsubsection{Linked (Open) Data}

Laut W3C ist das Prinzip von Linked Data das Herzstück des semantischen Webs. Im semantischen Web sollen auch Beziehungen zwischen Daten zur Verfügung gestellt werden. So wird aus einer reinen Sammlung von Datensätze ein Web of Data. ${ }^{12}$

\footnotetext{
${ }^{6}$ Vgl. o.V. (o.J.): World Wide Web, https://www.gruenderszene.de/lexikon/begriffe/world-wide-web?interstitial, Zugriff am 29.11.2019.

${ }^{7}$ Vgl. Hitzler et al. (2008), S. 10.

${ }^{8}$ Hitzler et al. (2008), S. 1.

${ }^{9}$ Hitzler et al. (2008), S. 1.

${ }^{10}$ Hitzler et al. (2008), S. 12.

${ }^{11}$ Vgl. World Wide Web Consortium (2019): About W3C, https://www.w3.org/Consortium/, Zugriff am 29.11.2019.

12 Vgl. World Wide Web Consortium (2015): Linked Data,

http://www.w3.org/standards/semanticweb/data, Zugriff am 30.12.2019.
} 
Tim Berners-Lee empfiehlt die Einhaltung folgender vier Regeln beim Umgang mit Linked Data:

1. Verwendung von URIs als Bezeichnungen für Objekte,

2. Verwendung von HTTP-URIs, um die Bezeichnungen nachschlagen zu können,

3. Bereitstellung brauchbarer standardisierter Informationen (RDF, SPARQL), für den Fall, dass eine Bezeichnung nachgeschlagen wird,

4. Hinzufügen weiterer Links auf andere URIs zu den Informationen, sodass neue Objekte entdeckt werden können. ${ }^{13}$

Sowohl beim World Wide Web als auch bei dessen Erweiterung, dem semantischen Web, wird das Hypertext Transfer Protocol (HTTP) als Zugriffmechanismus auf andere Ressourcen verwendet.

Im Gegensatz zum Web, bei dem zum Beispiel Hypertext Markup Language (HTML) Dokumente über Links auf HTTP-Uniform Resource Identifier (URI) miteinander in Beziehung gesetzt werden, werden bei Linked Data HTTP-URIs zur eindeutigen Identifizierung (Regel 1) und zur Dereferenzierung (Regel 2) von Ressourcen verwendet. Das bedeutet, dass der URI auch zu einer Beschreibung einer Ressource führen soll. ${ }^{14}$

Mit Hilfe von W3C Standards wie dem Resource Description Framework (RDF), dem Resource Description Framework Schema (RDFS) und der Web Ontology Language (OWL) ist es möglich, Beziehungen zwischen Ressourcen maschinenlesbar zu machen (Regel 3 und 4).

Wie bereits erwähnt, ist W3C das wichtigste Gremium, wenn es um die Entwicklung von Webstandards geht. Webstandards sind technische Spezifikationen und Richtlinien, die eingehalten werden sollen, um zum Beispiel die Funktion standardkonformer Webseiten auf verschiedenen Browsern und Geräten sowie eine hohe technische Qualität zu gewährleisten. ${ }^{15}$

Für das semantische Web ermöglicht die Verwendung gemeinsamer und offener Standards auch die Implementierung von Software-Applikationen, die über einen gemeinsamen Linked DataInformationsraum operieren und komplexe Zusammenhänge zwischen Ressourcen entdecken und auswerten können. ${ }^{16}$ Ein wichtiges Stichwort in diesem Zusammenhang ist das Reasoning. Der Begriff wird unter 2.1.4 noch genauer definiert.

\subsubsection{Standards im semantischen Web}

Im Folgenden werden die für die Arbeit wichtigen Standards des semantischen Webs genauer beschrieben.

\section{Resource Description Framework (RDF)}

„Das Resource Description Framework (...) ist eine formale Sprache für die Beschreibung strukturierter Informationen. ${ }^{17}$

\footnotetext{
${ }^{13}$ Vgl. Berners-Lee, T. (2006): Linked Data, http://www.w3.org/Designlssues/LinkedData.html, Zugriff am 30.12.2019.

${ }^{14} \mathrm{Vgl}$. Gradmann et al. (2012), S. 19.

${ }^{15} \mathrm{Vgl}$. World Wide Web Consortium (2019): Standards, http://www.w3.org/standards/, Zugriff am

31.12.2019.

${ }^{16} \mathrm{Vgl.}$ Gradmann et al. (2012), S. 20.

${ }^{17}$ Hitzler et al. (2008), S. 35.
} 
Dabei handelt es sich um ein einfaches Graphen-basiertes Datenmodell für die Modellierung von Aussagen über Ressourcen (URI-Bezeichner): Ein RDF-Dokument beschreibt einen gerichteten Graphen. Ein gerichteter Graph ist eine Menge von Knoten, die durch gerichtete Kanten miteinander verbunden werden. Knoten und Kanten haben eindeutige Bezeichner. ${ }^{18}$ Man spricht auch von Tripeln. Ein Tripel besteht aus Subjekt, Prädikat und Objekt. Ein Subjekt ist immer eine Ressource, die über eine semantische Beziehung (Prädikat) mit einem Objekt verbunden ist. Das Objekt kann hierbei eine weitere Ressource oder ein Literal sein. ${ }^{19}$ Die folgende Abbildung 1 veranschaulicht den Aufbau eines RDF-Graphen:

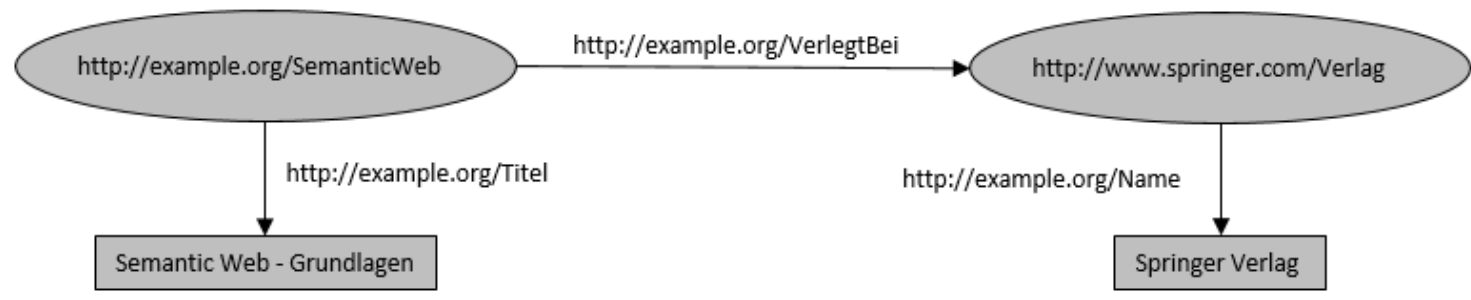

Abbildung 1: Ein RDF-Graph mit Literalen zur Beschreibung von Datenwerten.

Quelle: Hitzler, P. / Krötzsch, M. / Rudolph, S. / Sure, Y. (2008): Semantic Web, 1. Auflage, Berlin: Springer, S. 39 .

Aus der Abbildung 1 geht hervor, dass das Buch Semantic Web mit dem Titel Semantic WebGrundlagen beim Verlag mit dem Namen Springer Verlag verlegt wird. Ressourcen bzw. URIs werden in Ellipsen dargestellt, Literale in Rechtecken. An dieser Stelle ist es noch einmal wichtig zu erwähnen, dass die URIs erstmal nur zur eindeutigen Bezeichnung eines Objektes bzw. einer Ressource dienen, diese müssen nicht unbedingt online abrufbar sein.

Literale beschreiben Datenwerte, also reservierte Bezeichner für RDF-Ressourcen. Diese haben einen bestimmten Datentyp. Wenn, wie in Abbildung 1, kein Datentyp angegeben ist, handelt es sich um eine Zeichenkette.

Die durch RDF beschriebenen Graphen können auch durch Zeichenketten syntaktisch dargestellt werden. Dies ist durch die bereits oben genannten Tripel möglich. Es gibt verschiedene Enkodierungen zur Darstellung eines RDF Graphen: Notation 3, JSON-LD, Turtle, RDF/XML und andere. Die Umwandlung des Graphen in eine Zeichenkette nennt man auch Serialisierung. ${ }^{20}$ In dieser Arbeit wird nur auf die RDF/XML-Serialisierung genauer eingegangen.

\section{RDF/XML-Serialisierung:}

Wie der Name schon sagt ist RDF/XML eine XML-basierte Schreibweise.

Die Extensible Markup Language (XML) ist eine Sprache zur Beschreibung und zum Austausch hierarchisch strukturierter Daten, die von Menschen und auch von Maschinen lesbar ist. ${ }^{21}$

Der Graph aus Abbildung 1 kann nun wie folgt in RDF/XML beschrieben werden:

\footnotetext{
${ }^{18}$ Vgl. Hitzler et al. (2008), S. 36.

${ }^{19} \mathrm{Vgl.} \mathrm{Gradmann}$ et al. (2012), S. 19.

${ }^{20} \mathrm{Vgl}$. Hitzler et al. (2008), S.38 ff.

${ }^{21}$ Vgl. World Wide Web Consortium (2015): XML Essentials, http://www.w3.org/standards/xml/core,

Zugriff am 01.01.2020. Weitere Informationen zu XML auf der Webseite.
} 


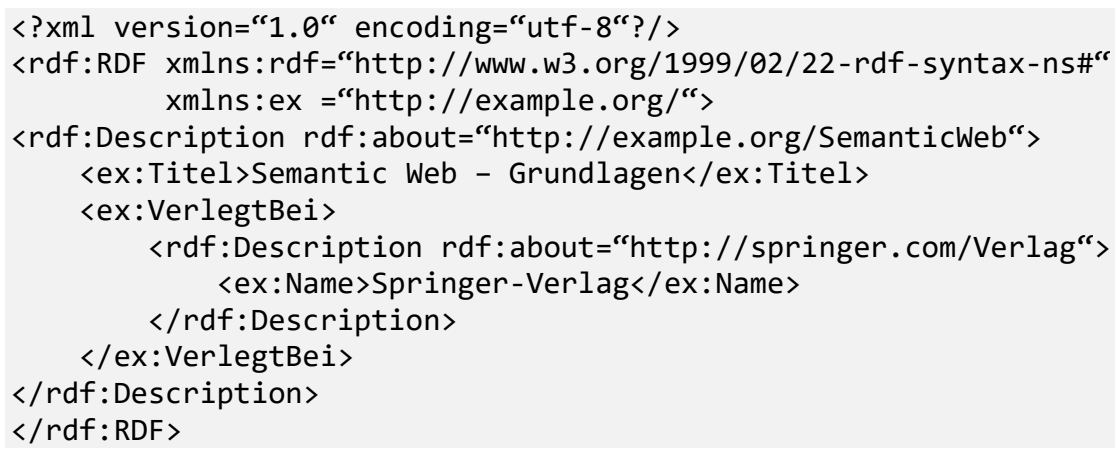

In der ersten Zeile befinden sich Angaben über die XML-Version und die -Kodierung. Der darauffolgende Knoten rdf:RDF ist die Wurzel des RDF/XML-Dokumentes. Anschließend werden die Namensräume deklariert. Die Präfixe $r d f$ und ex sind beliebige Abkürzungen für eine übersichtlichere Angabe der Namensräume im Dokument. Nachfolgend werden die Tripel kodiert. Subjekte und Objekte sind durch Elemente des Typs rdf:Description gekennzeichnet, das XML-Attribut rdf:about gibt hierbei den Bezeichner des Elements an. Prädikate werden direkt als Elemente angegeben. Literale werden einfach als Inhalte eines Prädikats-Elements dargestellt. Zuletzt wird der Knoten rdf: RDF zum Schließen des RDF-Dokumentes angegeben. ${ }^{22}$

Die oben gezeigte Darstellung ist allerdings nicht die einzige Möglichkeit, wie der Graph in $\mathrm{RDF} / \mathrm{XML}$ beschrieben werden kann:

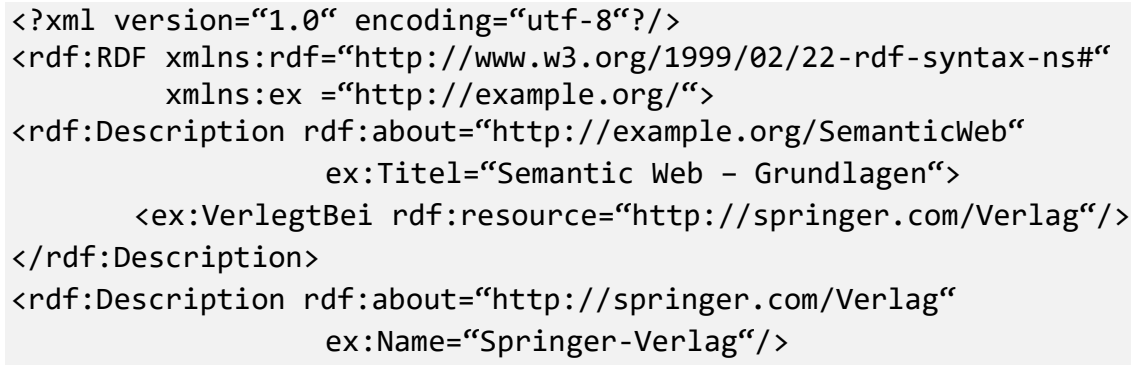

Bei dieser Schreibweise sind alle Prädikate mit Literalen als Attribute kodiert (für URIs wäre dies nicht möglich). Weiterhin lässt sich mit dem Attribut rdf: resource direkt das Objekt eines Tripels angeben, sodass kein untergeordnetes Element vom Typ rdf:Description nötig ist. Da jeder Wert von rdf:resource als URI interpretiert wird, ist die Schreibweise nicht für Literale geeignet. ${ }^{23}$ Die Beispiele zeigen, dass die Darstellung von RDF in XML sehr vielfältig ist, was vor allem daran liegt, dass die gleichen Tripel unterschiedlich kodiert werden können. Trotzdem beschreiben die Tripel am Ende alle denselben RDF-Graphen. ${ }^{24}$

\section{Resource Description Framework Schema (RDFS)}

Mit Hilfe von RDF lassen sich also einfache Aussagen über einzelne Ressourcen machen und es lässt sich Wissen verknüpfen. Resource Description Framework Schema (RDFS) erweitert RDF, indem es eine extern spezifizierte Semantik einführt. Mit RDFS lassen sich spezielle Klassen (Dinge mit ähnlichen Eigenschaften), deren Eigenschaften und die Beziehungen zwischen diesen

${ }^{22}$ Vgl. Hitzler et al. (2008), S.43 ff.

${ }^{23}$ Vgl. Hitzler et al. (2008), S.44.

${ }^{24} \mathrm{Vgl}$. Hitzler et al. (2008), S.45. 
(Properties) beschreiben. In dieser Arbeit wird auch der Begriff Property verwendet, da eine einfache Übersetzung als Eigenschaft nicht vollständig korrekt wäre. ${ }^{25}$ Tabelle 1 erläutert die wichtigsten Klassen in $\operatorname{RDF}(\mathrm{S})$ :

Tabelle 1: Die wichtigsten Klassen in RDF und RDFS.

Quelle: Eigene Darstellung in Anlehnung an Dengel, A. (Hrsg.) (2012): Semantische Technologien. Grundlagen - Konzepte - Anwendungen, 1. Auflage, Heidelberg: Spektrum Akademischer Verlag, S. $124 \mathrm{f}$.

\begin{tabular}{|l|l|}
\hline Klasse & Beschreibung \\
\hline rdfs:Resource & Ableitung aller Ressourcen. \\
\hline rdfs:Class & Auszeichnung einer Ressource als Klasse. \\
\hline rdf:Property & Ressource wird zur Property. \\
\hline
\end{tabular}

Tabelle 2 veranschaulicht die bedeutsamsten vordefinierten Properties in RDF(S):

Tabelle 2: Die wichtigsten vordefinierten Properties in RDF und RDFS.

Quelle: Eigene Darstellung in Anlehnung an Dengel, A. (Hrsg.) (2012): Semantische Technologien. Grundlagen - Konzepte - Anwendungen, 1. Auflage, Heidelberg: Spektrum Akademischer Verlag, S. 125.

\begin{tabular}{|l|l|}
\hline Property & Beschreibung \\
\hline rdf:type & Erzeugt eine Instanz einer Klasse. \\
\hline rdfs: subclassof & Bildung von Unterklassen einer Klasse. \\
\hline rdfs: subPropertyof & Spezialisierung von Properties. \\
\hline
\end{tabular}

Zudem gibt es zwei Möglichkeiten, um Einschränkungen, also sogenannte Constraints, zu bestimmen. Diese sind in Tabelle 3 aufgeführt:

Tabelle 3: Einschränkungen in RDFS.

Quelle: Eigene Darstellung in Anlehnung an Dengel, A. (Hrsg.) (2012): Semantische Technologien. Grundlagen - Konzepte - Anwendungen, 1. Auflage, Heidelberg: Spektrum Akademischer Verlag, S. 125.

\begin{tabular}{|l|l|}
\hline Constraint & Beschreibung \\
\hline rdfs:range & $\begin{array}{l}\text { Einschränkung des Wertebereichs / Range-Constraint. } \\
\text { Schränkt Wertemenge einer Property ein. }\end{array}$ \\
\hline \multirow{3}{*}{ rdfs:domain } & $\begin{array}{l}\text { Einschränkung des Definitionsbereichs / Domain- } \\
\text { Constraint. Schränkt Menge der Klassen ein, auf die eine } \\
\\
\text { Property angewendet werden darf. }\end{array}$ \\
\hline
\end{tabular}

„Einen Satz von Bezeichnern für Individuen, Beziehungen und Klassen nennt man (...) Vokabular. ${ }^{126}$ RDFS ist in diesem Fall ein spezielles RDF-Vokabular. Das bedeutet auch, dass RDFS kein thematisches Vokabular ist. In einem thematischen Vokabular werden neue Termini für eine spezielle Anwendungsdomäne bereitgestellt. RDFS dagegen stellt universelle Ausdrucksmittel bereit, „(...) welche ermöglichen, innerhalb eines RDFS-Dokuments Aussagen über die semantischen Beziehungen der Termini eines beliebigen nutzerdefinierten Vokabular zu machen. ${ }^{27}$ RDFS ist somit eine Ontologiesprache, mit der bereits einfache thematische Ontologien beschrieben werden können. Da aber auch RDFS Grenzen hat, basieren einige anspruchsvollere Ontologien auf der Web Ontology Language.

${ }^{25}$ Vgl. Dengel, A. (Hrsg.) (2012): Semantische Technologien. Grundlagen - Konzepte - Anwendungen, 1. Auflage, Heidelberg: Spektrum Akademischer Verlag, S. 124.

${ }^{26}$ Hitzler et al. (2008), S.66.

${ }^{27}$ Hitzler et al. (2008), S.67. 


\section{Web Ontology Language (OWL)}

Die Web Ontology Language (OWL) ist eine erweiterte Ontologiesprache der zuvor beschriebenen Standards. Zum Beispiel sind die folgenden Ausdrucksmöglichkeiten erst in OWL möglich:

- Lokalisierung von Domain- und Range-Constraints, das heißt, es können Einschränkungen deklariert werden, die nur für bestimmte Klassen gelten.

o Hinzufügen von Existenz- / Kardinalitäts-Constraints, das heißt, es können Einschränkungen deklariert werden, die angeben, wie viele unterschiedliche Werte eine Property annehmen darf oder muss.

○ Hinzufügen von Eigenschaften der Transitivität (z.B. isPartof), Symmetrie (z.B. berührt) und des Inversen (z.B. hasPart ist das Inverse zu isPartof). ${ }^{28}$

Eine mögliche Syntax von OWL ist RDF basiert. In dieser Arbeit wird sich auch nur auf diese Syntax bezogen. Auch in OWL gibt es Klassen und Properties. Die owl:Class ist eine Unterklasse der rdfs:Class und die owl:ObjectProperty bzw. die owl:DatatypeProperty sind Unterklassen der rdf:Property. ${ }^{29}$

Mittlerweile gibt es mit OWL 2 eine neue Version der Ontologiesprache OWL. Das heißt nicht, dass Ontologien in OWL 1 ihre Gültigkeit verlieren. OWL 2 fügt dagegen neue Funktionen in Bezug auf OWL 1 hinzu. ${ }^{30}$ Diese sollen an dieser Stelle aber nicht weiter vertieft werden.

\subsubsection{Ontology-Management}

Zum Ontology-Management gehört unter anderem die Möglichkeiten der Entwicklung von Ontologien. Diese sind für diese Arbeit allerdings nicht weiter relevant. Weiterhin kommt es während der Arbeit mit Ontologien oder bei der Entwicklung von Anwendungen vor, dass verschiedene Ontologien verwendet werden. Bei der Kombination von Ontologien unterscheidet man die Vereinigung von Ontologien (Ontology-Merging) und die Zuordnung (Ontology-Alignment / -Mapping). Ontology-Alignments „(..) sind Aussagen über Beziehungen zwischen den Elementen zweier Ontologien (...). “31 Die Ontologien werden sozusagen aufeinander abgebildet.

\subsubsection{Anfragesprachen und Reasoning}

Ein wichtiges Ziel des Semantic Web ist es, Wissen aus den neuen Ontologien zu ziehen. Anfragesprachen wie die XML Path Language (XPATH) und die SPARQL Protocol and RDF Query Language dienen dazu, Wissen aus eben diesen Wissensbasen abzufragen. Für OWL sind Abfragesprachen nur bedingt geeignet. Mit Hilfe von Reasoning kann neues Wissen aus den Wissenbasen hergeleitet werden. ${ }^{32}$

\footnotetext{
${ }^{28} \mathrm{Vgl}$. Dengel (2012), S. 127.

${ }^{29}$ Vgl. Antoniou, G. \& Harmelen, F. (2003): Web ontology Language: OWL. In: Staab S. \& Studer R. (Hrsg.): Handbook on Ontologies, S. 67 ff., http://doi.org/10.1007/978-3-540-92673-3_4, Zugriff am 04.01.2020.

${ }^{30}$ Vgl. World Wide Web Consortium (2012): OWL 2 Web Ontology Language. Document Overview (Second Edition), http://www.w3.org/TR/2012/REC-ow/2-overview-20121211/, Zugriff am 04.01.2020.

${ }^{31}$ Dengel (2012), S. 138.

32 Vgl. Dengel (2012), S. 161.
} 


\section{XML Path Language (XPATH)}

Die XML Path Language (XPATH) ist eigentlich eine von W3C, nicht speziell für das semantische Web entwickelte Abfragesprache zum Navigieren durch Elemente und Attribute eines XML Dokumentes. Allerdings können RDF/XML Dokumente auch mit XPATH abgefragt werden, da diese in ihrer Struktur auf XML basieren. Ein XPATH-Ausdruck ähnelt einer Pfadangabe in einem Computer-Datei-System. Zudem unterstützt XPATH Funktionen für Strings, numerische, boolesche und andere Werte. ${ }^{33}$

\section{SPARQL Protocol and RDF Query Language}

Die SPARQL Protocol and RDF Query Language ist eine W3C Empfehlung für die Abfrage von in RDF spezifizierten Informationen und die Darstellung der daraus resultierenden Ergebnisse. SPARQL basiert auf einfachen RDF-Anfragen in Form von Graphenmustern, es enthält aber auch erweiterte Funktionen für die Konstruktion komplexerer Anfragemuster, für die Verwendung von Filterbedingungen und die Ausgabeformatierung. ${ }^{34}$

\section{Reasoning}

„Im Unterschied zu Anfragesprachen erlaubt echtes Reasoning ein deutlich komplexeres Herleiten von neuem Wissen aus Wissenbasen. ${ }^{135}$ Dabei werden sogenannte Regelsprachen verwendet. Hierbei können die einzelnen Anfragen verkettet oder ineinander eingesetzt werden. Durch die zusätzliche Verwendung von Rekursion erzielt man so eine höhere Ausdrucksmächtigkeit. ${ }^{36}$

\subsection{Webintegrierung von Sensoren und Sensordaten}

Im Folgenden wird kurz erläutert, welche aktuellen Möglichkeiten es zur Integrierung von Sensoren und Sensordaten in das Web gibt.

\subsubsection{Das Sensorweb Enablement (SWE)}

"Das Sensor Web Enablement (SWE) ist eine Initiative des Open Geospatial Consortium (OGC). "137 OGC ist ein internationales Konsortium aus Unternehmen, Regierungsbehörden, Forschungsorganisationen und Universitäten, dessen Ziel es ist, Informationen und Dienste für Geodaten auffindbar, zugänglich, interoperabel und wiederverwendbar zu machen. ${ }^{38}$

Durch SWE werden Interoperabilitätsschnittstellen und Metadatenkodierungen spezifiziert zur Echtzeitintegration heterogener Sensornetzwerke und des Internet of Things (IoT) in die

\footnotetext{
${ }^{33} \mathrm{Vgl}$. Refsnes Data (o.J.): XPath Tutorial, https://www.w3schools.com/xml/xpath_intro.asp, Zugriff am 04.01.2020.

${ }^{34} \mathrm{Vgl}$. Hitzler et al. (2008), S.202.

${ }^{35}$ Dengel (2012), S. 170.

${ }^{36} \mathrm{Vgl.}$ Dengel (2012), S. 170.

${ }^{37}$ GIS-Technologie, Interoperabilität, IT, Standards (o.J.): SensorWeb, http://www.gis-news.de/technologie/sensorweb/, Zugriff am 04.01.2020.

${ }^{38} \mathrm{Vgl}$. Open Geospatial Consortium (2020): Welcome to The Open Geospatial Consortium, https://www.opengeospatial.org/, Zugriff am 04.01.2020.
} 
Informationsinfrastruktur. ${ }^{39}$ IoT ist das Netzwerk von physisches Objekten, die in Sensoren, Software und anderen Technologie integriert sind zur Verknüpfung und zum Austausch von Daten mit anderen Geräten und Systemen über das Internet. ${ }^{40}$

Die Sensor Model Language (SensorML) ist ein XML-Schema zur Beschreibung von Sensorsystemen und -prozessen im Zusammenhang mit Sensorbeobachtungen. Der Sensor Observation Service (SOS) ist eine Standard-Web-Service-Schnittstelle zum Anfordern, Filtern und Abrufen von Beobachtungen und Sensorsysteminformationen. ${ }^{41}$

\subsubsection{Das semantische Sensorweb (SSW)}

Das SWE ist in erster Linie nicht auf das semantische Web ausgerichtet. SWE dient aber als Basis für die Entwicklung eines semantischen Sensorwebs (SSW). Durch Hinzufügen semantischer Annotationen zu vorhandenen Standardsensorsprachen des SWE wird die Bedeutung der Sensoren und ihren Beobachtungen erweitert. Dadurch wird auch der Zugriff auf Sensordaten verbessert. Dabei wird auf die Technologien des semantischen Webs zurückgegriffen wie die Erstellung von Ontologien basierend auf RDFS oder OWL zur Beschreibung von Sensoren und Sensordaten. ${ }^{42}$

\subsection{Cloud-Computing}

Bei Cloud-Computing werden Cloud-Ressourcen über das Internet bereitgestellt, über die Entwickler ihre Anwendungen laufen und zur Verfügung stellen lassen können. Zu den Ressourcen gehören beispielsweise Server, Speicher, Datenbanken, Netzwerkkomponenten, Software und Analysefunktionen.

Durch Cloud-Computing können so Kosten gesenkt werden, da keine Hardware oder Software direkt erworben werden muss, keine lokalen Rechnerzentren betrieben werden müssen (inklusive Stromversorgung und Kühlung) sowie keine IT-Experten zur Verwaltung der Infrastruktur eingestellt werden müssen. Stattdessen muss der Entwickler meist nur für die Cloud-Dienste bezahlen, die er auch tatsächlich verwendet. Zudem lassen sich große Mengen an Cloud-Ressourcen in wenigen Minuten mit wenigen Klicks bereitstellen, wobei die Ressourcen selbst flexibel skaliert werden können.

Weiterhin gibt es unterschiedliche Arten von Cloud-Computing. In dieser Arbeit soll eine öffentliche Cloud von einem öffentlichen Anbieter verwendet werden. Hierbei sind die Infrastrukturkomponenten Eigentum des Cloud-Anbieters und werden auch von diesem verwaltet. Auf die Dienste wird über einen Webbrowser zugegriffen. Der Cloud-Dienst selbst soll serverless (dt. serverlos) sein, das heißt, die Infrastruktur, die zur Ausführung des Codes erforderlich ist, wird vom Cloud-Dienstanbieter automatisch bereitgestellt, skaliert und verwaltet. Die Skalierung wird dabei an die Anforderungen des Workloads angepasst. ${ }^{43}$

\footnotetext{
${ }^{39} \mathrm{Vgl}$. Open Geospatial Consortium (2020): Sensor Web Enablement DWG, https://www.opengeospatial.org/projects/groups/sensorwebdwg, Zugriff am 04.01.2020.

${ }^{40} \mathrm{Vgl}$. Oracle (2019): Was ist das Internet of Things?, https://www.oracle.com/de/internet-of-things/what-is-iot.html, Zugriff am 04.01.2020.

${ }^{41}$ Vgl. Open Geospatial Consortium (2020).

42 Vgl. Shet et al. (2008), S. 80.

${ }^{43}$ Vgl. Microsoft (2020): Was ist Cloud Computing?,

https://azure.microsoft.com/de-de/overview/what-is-cloud-computing/, Zugriff am 20.01.2020.
} 


\section{Das GEO Label}

Im Folgenden wird beschrieben, welche Gründe zur Entwicklung eines GEO Labels geführt haben und wie die Visualisierung des Labels aussieht. Zudem wird auf den aktuellen Stand der Java GEO Label API von 52 North $^{44}(52 \mathrm{~N})$ eingegangen. Die Abkürzung API steht für Anwendungsschnittstelle (engl. Application Programming Interface).

\subsection{Entwicklung}

Die Group on Earth Observation (GEO) ist ein globales Netzwerk, das Regierungsinstitutionen, Hochschul- und Forschungseinrichtungen, Datenanbieter, Unternehmen, Ingenieure, Wissenschaftler und Experten zusammenbringt, um über nationale Grenzen hinaus innovative Lösungen für globale Herausforderungen in einer Zeit des exponentiellen Datenwachstums, der menschlichen Entwicklung und des Klimawandels zu schaffen. ${ }^{45}$ Dabei entwickelt die GEO Community unter anderem das Global Earth Observation System of Systems (GEOSS). „GEOSS is a set of coordinated, independent Earth observation, information and processing systems that interact and provide access to diverse information for a broad range of users in both public and private sectors." ${ }^{\prime \prime 6}$

GEOSS bietet Zugang zu Millionen von Erdbeobachtungsdaten. Um den Nutzer bei der Suche nach geeigneten Datensätzen zu unterstützen, hat das GEO Science and Technologie Committee (STC) 2009 die Entwicklung eines GEO Labels vorgeschlagen. Dieses soll Informationen zur Datensatzqualität zusammenfassen und so darstellen, dass der Benutzer die Relevanz eines Datensatzes für seine Anforderungen leicht beurteilen kann. Die Umsetzung erfolgte durch das 7. Forschungsrahmenprogramm (FP7) Projekt GeoViQua (Quality aware Visualization for the Global Earth Observation System of Systems), ein Förderprogramm der Europäischen Kommission.

Aktuell ist das GEO Label noch nicht in GEOSS integriert. Stattdessen wurde mit dem Open Source Software Projekt GEO-label-java ${ }^{47}$ ein in Java geschriebener Prototyp für einen GEO Label Service von 52 North entwickelt. Dieser Service implementiert eine GEO Label API, die die Generierung von Labels ermöglicht. 2015 wurde die Implementierung von Nüst et al. speziell für Metadaten des Sensorwebs erweitert. ${ }^{48}$

Zudem gibt es mittlerweile zwei Versionen des GEO Labels, die sich in ihren Facetten (Informationsfeldern) teilweise unterscheiden. Da aber die GEO Label API, die als Basisimplementierung für diese Arbeit dienen soll, auf der ersten Version des Labels basiert, wird auf die zweite Version (GEO label for GEOSS 10 Data Managements Principles ${ }^{49}$ ) nicht weiter eingegangen. Die nachfolgend beschriebene Visualisierung bezieht sich auf die erste Version des GEO Labels.

\footnotetext{
${ }^{44}$ Weiterführende Informationen verfügbar unter: https://52north.org/.

${ }^{45} \mathrm{Vgl}$. Group on Earth Observations (o.J.a): GEO Community, https://www.earthobservations.org/geo_community.php, Zugriff am 16.11.2019.

${ }^{46}$ Group on Earth Observations (o.J.b): GEOSS, https://www.earthobservations.org/geoss.php, Zugriff am 20.11.2019.

${ }^{47}$ Verfügbar unter: https://github.com/52North/GEO-label-java.

${ }^{48}$ Nüst \& Lush (2015).

${ }^{49}$ Verfügbar unter: $h t t p: / / g e o l a b e l . i n f o /$.
} 


\subsection{Visualisierung}

Das GEO Label besteht aus acht unterschiedlichen Facetten, die Auskunft über das Vorhandensein unterschiedlicher Informationen für einen Datensatz geben. Die Facetten werden in Tabelle 5 genauer erläutert:

Tabelle 4: Symbole und Erläuterungen zu den einzelnen Facetten des GEO Labels.

Quelle: Eigene Darstellung in Anlehnung an Group on Earth Observations (o.J.): About, http://www.geolabel.info/About.htm, Zugriff am 21.11.2019.

\begin{tabular}{|c|c|c|}
\hline Information & Symbol & Erläuterung \\
\hline Producer Profile & & $\begin{array}{l}\text { Verfügbarkeit von (Kontakt-)Informationen zum Hersteller } \\
\text { eines Datensatzes (z.B. Organisation, Person). }\end{array}$ \\
\hline Lineage Information & & $\begin{array}{l}\text { Verfügbarkeit von Informationen über die Herkunft / } \\
\text { Verarbeitung der Daten (z.B. Anzahl der Bearbeitungs- / } \\
\text { Prozessierungsschritte). }\end{array}$ \\
\hline Producer Comments & & $\begin{array}{l}\text { Verfügbarkeit von informierenden Kommentaren vom } \\
\text { Hersteller über die Qualität der Daten (z.B. Probleme, } \\
\text { Vorschläge zur Nutzung). }\end{array}$ \\
\hline $\begin{array}{l}\text { Compliance with } \\
\text { Standards }\end{array}$ & & $\begin{array}{l}\text { Verfügbarkeit von Informationen über die Einhaltung des } \\
\text { Datensatzes von internationalen Standards (z.B. ISO 19115, } \\
\text { Dublin Core). }\end{array}$ \\
\hline Quality Information & & $\begin{array}{l}\text { Verfügbarkeit formaler Qualitätsmaße des Datensatzes (z.B. } \\
\text { Fehlermaße, Genauigkeitsangaben). }\end{array}$ \\
\hline User Feedback & & $\begin{array}{l}\text { Verfügbarkeit von Feedback, Kommentaren und } \\
\text { Bewertungen, die von den Benutzern des Datensatzes } \\
\text { bereitgestellt werden (z.B. allgemeine Kommentare zur } \\
\text { Datensatzqualität, erkannte Probleme, vorgeschlagene } \\
\text { Verwendung des Datensatzes). }\end{array}$ \\
\hline Expert Reviews & & 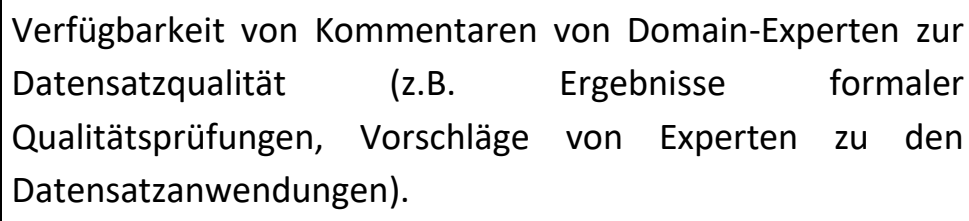 \\
\hline Citations Information & 2 & $\begin{array}{l}\text { Verfügbarkeit von Zitaten, bei denen der Datensatz } \\
\text { verwendet und zitiert wurde (z.B. formale Berichte zu } \\
\text { Datensatzqualitätsprüfungen, Zeitschriftenartikeln). }\end{array}$ \\
\hline
\end{tabular}

Bei der Erstellung eines Labels nimmt jede Facette jeweils einen von drei möglichen Verfügbarkeitszuständen an: Informationen sind verfügbar (available), Informationen sind auf einer höheren Ebene verfügbar (available at a higher level), Informationen sind nicht verfügbar (not available). Auf einer höheren Ebene bedeutet, dass die jeweilige Information für den gewählten Datensatz nicht vorliegt, für einen übergeordneten Datensatz allerdings schon. 
Die Unterscheidung der Verfügbarkeitszustände erfolgt über verschiedene visuelle Darstellungen, die in Abbildung 2 veranschaulicht werden:
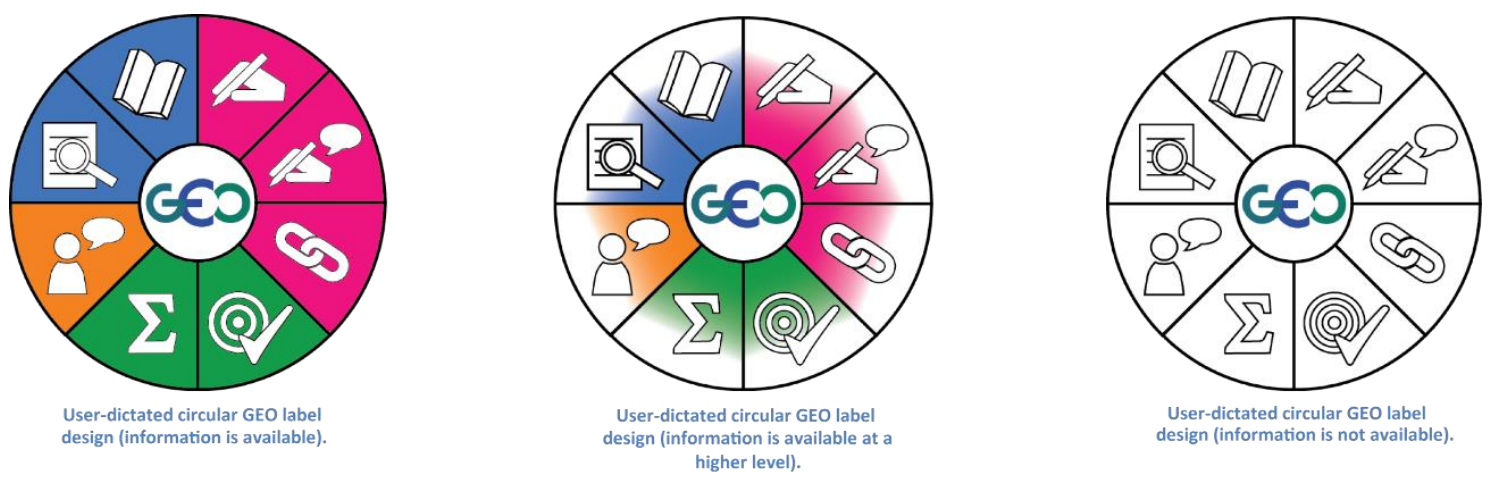

Abbildung 2: Visualisierung der Verfügbarkeitszustände der Facetten im GEO Label.

V.I.n.r.: Informationen sind verfügbar, Informationen sind auf einer höheren Ebene verfügbar, Informationen sind nicht verfügbar.

Quelle: Group on Earth Observations (o.J.): About, http://www.geolabel.info/About.htm, Zugriff am 21.11.2019.

Wenn eine Information verfügbar ist, wird die zugehörige Facette vollständig ausgefüllt. Wenn eine Information auf einer höheren Ebene verfügbar ist, wird die Facette zur Hälfte ausgefüllt. Wenn die Information nicht verfügbar ist, bleibt die Facette weiß. Die Facetten können natürlich unabhängig voneinander einen der drei Zustände annehmen. ${ }^{50}$

Weiterhin bietet das Label Hover-Over-Texte, um kurze zusammenfassende Informationen zu einer bestimmten Facette anzuzeigen, das heißt, wenn der Nutzer mit der Maus über eine Facette des generierten Labels fährt, erscheint ein kleiner Informationstext. In Abbildung 3 ist ein Hover-Over-Text für die Facette Producer Profile zu sehen, in dem die verantwortliche Organisation angegeben ist:

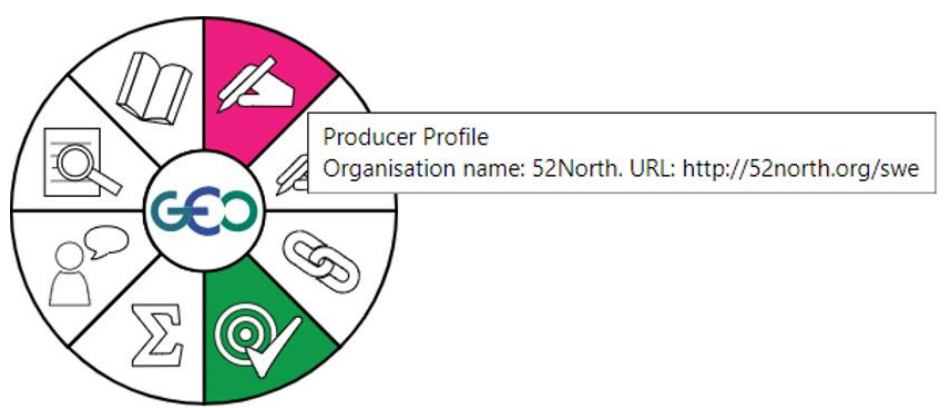

Abbildung 3: Beispiel für einen Hover-Over-Text für die Facette Producer Profile.

Quelle: Eigene Darstellung.

Neben der Hover-Over-Funktion existiert auch noch eine Drilldown-Funktion. Wenn der Nutzer auf eine Facette des generierten Labels klickt, werden für diese Facette strukturierte Informationen aus dem Metadatensatz, aus dem das Label generiert wurde, zurückgegeben. ${ }^{51}$

\footnotetext{
${ }^{50} \mathrm{Vgl.}$ Group on Earth Observations (o.J.c): About, http://www.geolabel.info/About.htm, Zugriff am 21.11.2019.

${ }^{51} \mathrm{Vgl}$. GeoViQua Consortium (2013): D.7.4 GCl pilot case perspective recommendations for enabling quality aware visualisation and search, http://www.geoviqua.org/Docs/SubmittedDeliverables/D7_4_GeoViQua.pdf, Zugriff am 21.11.2019.
} 


\subsection{Status Quo der Java GEO Label API}

Die Implementierung des 52 North Java GEO Label Service basiert auf Java 8. Es handelt sich hierbei um ein Maven-Multi-Module-Projekt ${ }^{52}$. Der Service beinhaltet die GEO Label API, die die Generierung von Labels auf Basis bestimmter XML-basierter Metadatendokumente erlaubt. Zu den unterstützten XML-basierten Metadatenstandards zählen hierbei ISO (International Organization for Standardization), FGDC (Federal Geographic Data Committee) und das GeoViQua Quality Model sowie speziell für das Sensorweb SensorML und SOS.

Grundlage der Generierung sind in JSON (JavaScript Object Notation) kodierte Transformationsdateien für die jeweiligen Metadatenformate. Diese enthalten XPATH Ausdrücke (es wird nur XPATH 1.0 unterstützt), um die Verfügbarkeit der Informationen für die Facetten in den Metadatendokumenten zu überprüfen und um die Hover-Over-Texte basierend auf einem String-Template zu erstellen. Zudem werden in den Dateien auch die URLs (Uniform Resource Locator) für die Drilldowns angegeben, die als Hyperlink den Facetten hinzugefügt werden. Die Software liest die Transformationsdateien und erstellt aus den enthaltenen XPATH Ausdrücken interne Objekte. Bei der Analyse eines Metadatendokumentes werden die Ausdrücke ausgewertet und anschließend wird aus den Ergebnissen ein Label auf Basis eines Template generiert. ${ }^{53}$

Aktuell wird die Möglichkeit, die Verfügbarkeit der Informationen für eine Facette als verfügbar auf einer höheren Ebene anzuzeigen, nicht unterstützt. Es sind aber Ansätze für die Umsetzung bereits im Code vorhanden.

Die Metadatendokumente können mit POST an den Server gesendet werden oder über eine GET URL angefordert werden. Die API ist verfügbar unter:

.../glbservice/api/v1

Es gibt mehrere Endpunkte zur Generierung von Labels:

.../glbservice/api/v1/svg (Label wird als Scalable Vector Graphic (SVG) zurückgegeben)

.../glbservice/api/v1/png (Label wird als Portable Network Graphics (PNG) zurückgegeben)

.../glbservice/api/v1/lml (Label wird als Label Markup Language (LML) zurückgegeben)

Als Parameter können jeweils metadata (Angabe des Metadatendokuments), feedback (Angabe eines externen Feedbackdokuments für die Feedback-Facetten) und size (Größe des Labels) verwendet werden.

Neben der GEO Label API existiert für den 52 North GEO Label Service noch eine Beispielseite (Abbildung in Anhang 1), auf der bereits Labels aus Beispieldokumenten generiert werden und auf der die Möglichkeit zur direkten Eingabe von einer Metadata-URL und/oder Feedback-URL zur Generierung eines Labels geboten wird:

${ }^{52}$ Weiterführende Informationen verfügbar unter:

https://maven.apache.org/guides/mini/guide-multiple-modules.html.

${ }^{53} \mathrm{Vgl}$. Nüst \& Lush (2015). 
4. Evaluation der Semantic Sensor Network Ontology und der verbundenen Ontologien zur Wahl der Informationsquellen für die Facetten des GEO Labels

.../glbservice

Zudem stellt der GEO Label Service noch eine Bearbeitungsseite (Abbildung in Anhang 2) bereit, auf der der Inhalt eines Metadatendokumentes in ein Textfeld übertragen und bearbeitet werden kann und anschließend ein Label aus dem Inhalt des Textfeldes generiert werden kann:

.../glbservice/editor.jsf

In dieser Arbeit wird der Fokus aber auf die 52 North GEO Label API gelegt. Da dieser Code als Basis für die Implementierung des Prototyps zur Generierung SSNO-basierter GEO Labels dient, müssen die RDF-Graphen, die die Sensorbeschreibungen modellieren, als RDF/XML serialisiert werden. So ist es möglich in der Implementierung weiterhin XPATH zu verwenden, um die Informationen für die Facetten des Labels abzufragen.

\section{Evaluation der Semantic Sensor Network Ontology und der verbundenen Ontologien zur Wahl der Informationsquellen für die Facetten des GEO Labels}

In diesem Kapitel werden die verschiedenen Ontologien evaluiert, um Informationsquellen für die Facetten des Labels ausfindig zu machen, die in einem RDF-Graphen über eine Sensorbeschreibung enthalten sein müssen. Abschließend wird die Wahl der endgültigen Informationsquellen mit den genauen Beschreibungen der gewählten Klassen und Properties noch einmal geordnet nach Facetten zusammengefasst. Tabelle 6 gibt bereits einen Überblick über die Namensräume und den zugehörigen Präfixen der Ontologien und Vokabulare, die im Folgenden erwähnt werden. Durch den Einsatz der Präfixe können die Klassen und Properties direkt den Ontologien und Vokabularen zugeordnet werden. Zudem geben die Namensräume an, unter welchen URLs die jeweiligen Ontologien und Vokabulare verfügbar sind.

Tabelle 5: Präfixe und Namensräume der erwähnten Ontologien und Vokabulare.

Quelle: Eigene Darstellung.

\begin{tabular}{|l|l|l|}
\hline Ontologie / Vokabular & Präfix & Namensraum \\
\hline Resource Description Framework & rdf & $\begin{array}{l}\text { http://www.w3.org/1999/02/22-rdf- } \\
\text { syntax-ns\# }\end{array}$ \\
\hline $\begin{array}{l}\text { Resource Description Framework } \\
\text { Schema }\end{array}$ & rdfs & $\begin{array}{l}\text { http://www.w3.org/2000/01/rdf- } \\
\text { schema\# }\end{array}$ \\
\hline Semantic Sensor Network Ontology & ssn & http://www.w3.org/ns/ssn/ \\
\hline $\begin{array}{l}\text { Sensor, Observation, Sampling and } \\
\text { Actuator }\end{array}$ & sosa & http://www.w3.org/ns/sosa/ \\
\hline System Capabilities Module & ssn-system & https://www.w3.org/ns/ssn/systems/ \\
\hline Provenance Interchange Ontology & prov & https://www.w3.org/ns/prov\# \\
\hline Friend of a Friend Ontology & foaf & http://xmlns.com/foaf/0.1/ \\
\hline Dataset Usage Vocabulary & duv & http://www.w3.org/ns/duv\# \\
\hline Data Catalog Vocabulary & dcat & https://www.w3.org/ns/dcat\# \\
\hline Bibliographic Reference Ontology & biro & http://purl.org/spar/biro/ \\
\hline Web Annotation Ontology & oa & http://www.w3.org/ns/oa\# \\
\hline
\end{tabular}




\subsection{Evaluation der Ontologien}

Für die Evaluation wird zunächst die Semantic Sensor Network Ontology (SSNO) beschrieben und es wird untersucht, inwieweit Informationsquellen aus dieser Ontologie für die Facetten des GEO Labels geeignet sind. Vorab wurden bereits Informationen und Daten zu SSNO von Professoren angefragt, die an der Erstellung der Ontologie beteiligt waren.

Anschließend müssen für die Facetten weitere Ontologien mit Informationsquellen gesucht werden, die nicht durch SSNO abgedeckt werden können. Die Suche erfolgt durch Recherche im Internet, wobei es sich besonders anbietet, bereits bestehende RDF-Graphen, die unter anderem Klassen und Properties aus SSNO beinhalten, zu betrachten. Dadurch lässt sich herausfinden, welche anderen Ontologien häufig mit der Semantic Sensor Network Ontology kombiniert werden. Zudem können auch neue Alignments zwischen SSNO und anderen Ontologien erstellt werden.

\subsubsection{Die Semantic Sensor Network Ontology}

Die ursprüngliche Version der Semantic Sensor Network Ontology ${ }^{54}$ wurde 2009 - 2011 von der W3C Semantic Sensor Network Incubator Group (SSN-XG) entwickelt und basiert auf OWL 2. Von 2015 - 2017 wurde die Ontologie von der W3C und OGC Spatial Data on the Web (SDW) Working Group überarbeitet und modularisiert. Das Modul SSN (Semantic Sensor Network) enthält nun die einfachere aber in sich geschlossene Kernontologie SOSA (Sensor, Observation, Sample and Actuator). ${ }^{55}$ Neben SSN und SOSA gibt es weitere Module, die zum Beispiel weitere Begriffe einführen oder die Beziehungen zu anderen Ontologien verdeutlichen (Ontology-Alignment). Diese sind in Abbildung 4 aufgeführt:

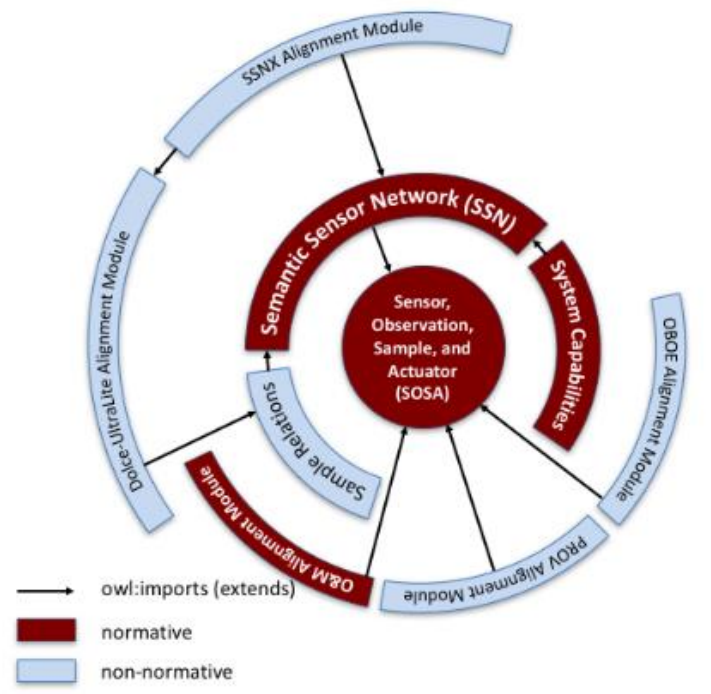

Abbildung 4: Die SOSA und SSN Ontologien und ihre vertikalen und horizontalen Module.

Quelle: Spatial Data on the Web Working Group (2017): Semantic Sensor Network Ontology, http://www.w3.org/TR/2017/REC-vocab-ssn-20171019/, Zugriff am 10.01.2020.

${ }^{54}$ Ontologie verfügbar unter: https://www.w3.org/2005/Incubator/ssn/ssnx/ssn.

${ }^{55}$ Vgl. Haller, A. / Janowicz, K. / Cox, S. / Lefransois, M. / Taylor, K. / Phuoc, D. / Liebermann, J. / GarciaCastro, R. / Atkinson, R. / Stadler, C. (2019): The Modular SSN Ontology: A Joint W3C and OGC Standard Specifying the Semantics of Sensors, Observations, Sampling, and Actuation. In: Semantic Web, Nr. 10, S. 9-33, http://armin-haller.com/publications/SSN-SWJ.pdf, Zugriff am 10.01.2020. 
4. Evaluation der Semantic Sensor Network Ontology und der verbundenen Ontologien zur Wahl der Informationsquellen für die Facetten des GEO Labels

Vertikal segmentierte SSN-Module importieren niedrigere Module direkt und definieren neue Axiome (wahre Aussagen innerhalb einer Ontologie). Die Module der niedrigeren Ebene sind unabhängig von den Modulen der höheren Ebene und für sich logisch konsistent. Zum Beispiel importiert SSN SOSA. SOSA selbst importiert keine andere Ontologie und kann auch alleinstehend verwendet werden. Horizontal geschichtete Module können voneinander abhängen, das heißt, sie können vom Import eines anderen horizontalen Moduls abhängen. Horizontale Module fügen jedoch nur Klassen und Eigenschaften hinzu, die Semantik der vorhandenen Begriffe wird nicht erweitert. ${ }^{56}$

"The Semantic Sensor Network (SSN) ontology is an ontology for describing sensors and their observations, the involved procedures, the studied features of interest, the samples used to do so, and the observed properties, as well as actuators. ${ }^{157}$

Die Konzepte von Observations, Actuations und Samplings sind eng miteinander verknüpft, die Klassen überlagern sich sozusagen. Deshalb können alle Drei mit der SSN-Ontologie beschrieben werden. Eine sosa:observation ist eine Beobachtung, die von einem sosa:Sensor gemacht wurde. Beobachtungen verwenden aber meist (Stich-)Proben. Um diese Proben bzw. die Probenahme zu beschreiben, wurden sosa:Sample und sosa:Samplings eingeführt. Ein sosa:Actuator (deutsch Aktuator) löst eine Betätigung, also eine sosa:Actuation aus, das heißt, dass zum Beispiel ein elektrisches Gerät beim Empfang eines Signals etwas in seiner Umgebung ändert. $^{58}$

Abbildung 5 führt alle Klassen und Eigenschaften von SSN (also einschließlich SOSA) aus der Perspektive der sosa:observation auf:

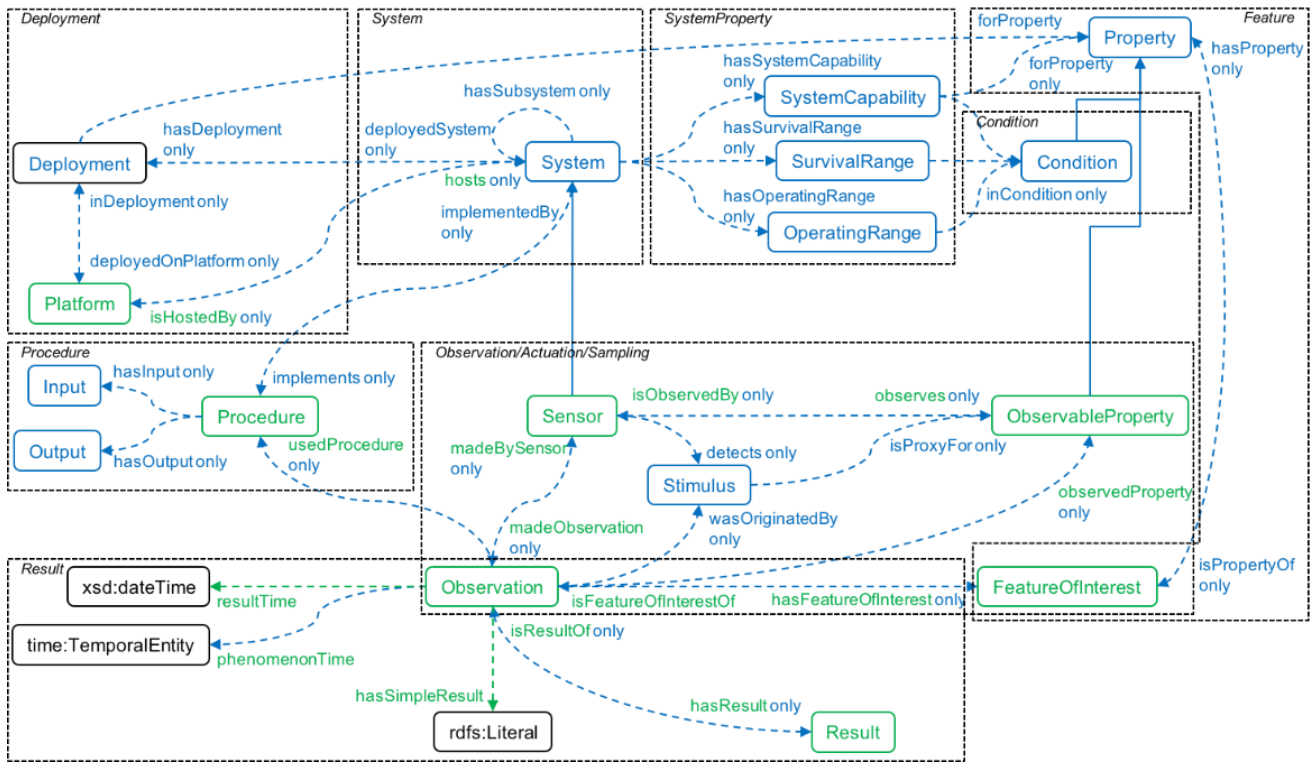

Abbildung 5: Übersicht über die SSN Klassen und Eigenschaften (Observation Perspektive). Grün: SOSA-Komponenten, Blau: nur SSN-Komponenten.

Quelle: Spatial Data on the Web Working Group (2017): Semantic Sensor Network Ontology, http://www.w3.org/TR/2017/REC-vocab-ssn-20171019/, Zugriff am 10.01.2020.

${ }^{56}$ Vgl. Haller et al. (2018), S. $7 \mathrm{ff}$.

57 Spatial Data on the Web Working Group (2017): Semantic Sensor Network Ontology, http://www.w3.org/TR/2017/REC-vocab-ssn-20171019/, Zugriff am 10.01.2020.

${ }^{58} \mathrm{Vgl}$. Haller et al. (2018), S. $11 \mathrm{ff}$. 
4. Evaluation der Semantic Sensor Network Ontology und der verbundenen Ontologien zur Wahl der Informationsquellen für die Facetten des GEO Labels

Anstatt aus Sicht der sosa: Observation, könnte die Abbildung 5 auch aus der Perspektive einer sosa:Actuation ${ }^{59}$ oder eines sosa:Sampling ${ }^{60}$ erstellt werden. Die Unterschiede in den Abbildungen wären nur gering und spielen für diese Arbeit keine Rolle, deshalb wird sich weiterhin nur auf die sosa:observation-Perspektive bezogen.

Aus Abbildung 5 geht hervor, dass SSN insbesondere auf die Beschreibung der technischen Hintergründe einer Observation spezialisiert ist. Darunter fällt beispielsweise das sosa:Procedure. Ein sosa:Procedure ist ein Arbeitsablauf, Protokoll, Plan, Algorithmus oder eine Rechenmethode, die angibt, wie zum Beispiel eine sosa:Observation oder eine sosa:Actuation gemacht wurde. Dies könnte auf die Facette Lineage Information angewendet werden.

Weiterhin lässt sich mit SSNO ein ssn: System modellieren, auf dem ein sosa: Sensor basiert. Die Eigenschaften des ssn:System werden mit dem horizontalen System Capabilities Modul eingefügt. Zu den Eigenschaften zählen zum Beispiel die Genauigkeit (ssn-system:Accuracy) oder die Präzision (ssn-system:Precision) aber auch die Lebensdauer eines Systems, also Informationen, die auf die Facette Quality Information bezogen werden können. ${ }^{61}$

Als nächstes können Beispieldaten ${ }^{62}$ auf der offiziellen Seite der Semantic Sensor Network Ontology untersucht werden. In diesen werden häufig Kommentare mit rdfs: comment in die RDF Graphen eingefügt. Diese Property aus RDFS wäre geeignet zur Abbildung auf die Facette Producer Comments. Weiterhin beinhalten die Namensräume der Ontologien oder Vokabulare, die von W3C stammen, immer die Zeichenfolge w3.org. Im Gegensatz zu den anderen XMLbasierten Standards wie SensorML gibt es für Linked Open Data keine festen Gremien oder Konsortien, die alle Ontologien als feste Standards herausgeben. Trotzdem kann die genannte Zeichenkette zur Ausfüllung der Facette Compliance with Standards verwendet werden.

Für die restlichen Facetten müssen andere Ontologien gewählt werden. Durch das vertikale PROV Alignement Module (siehe Abbildung 4) bietet es sich an, die Provenance Interchange Ontology (PROV-O) für die Angabe von Informationen für die Facette Producer Profile zu verwenden. Die Ontologie wird unter 4.1 .2 beschrieben. Für die Facetten User Feedback und Citation Information kann das Dataset Usage Vocabulary (DUV) angewendet werden. Dieses ist unter anderem über die Webseite Linked Open Vocabularies ${ }^{63}$ auffindbar, einer Seite für die Suche nach Ontologien und Vokabularen. DUV wird unter Kapitel 4.1.3 erläutert.

\subsubsection{Die Provenance Interchange Ontology}

Die W3C Provenance Interchange Ontology (PROV-O) basiert auch auf OWL2. Sie dient dazu, in verschiedenen Systemen und Kontexten Herkunftsinformationen darzustellen und auszutauschen. Die Herkunftsinformationen können mit Hilfe der Klassen und Properties von PROV-O beliebig detailliert dargestellt werden.

\footnotetext{
${ }^{59}$ Abbildung verfügbar unter: https://www.w3.org/TR/vocab-ssn/images/SSN-OntStructure-Actuation.png. ${ }^{60}$ Abbildung verfügbar unter: https://www.w3.org/TR/vocab-ssn/images/SSN-OntStructure-Sampling.png.

${ }^{61} \mathrm{Vgl}$. Spatial Data on the Web Working Group (2017).

62 Beispieldaten verfügbar unter: https://www.w3.org/TR/vocab-ssn/\#examples.

${ }^{63}$ Webseite verfügbar unter: https://lov.linkeddata.es/dataset/lov/.
} 
4. Evaluation der Semantic Sensor Network Ontology und der verbundenen Ontologien zur Wahl der Informationsquellen für die Facetten des GEO Labels

Es gibt drei Basisklassen: Die Klasse prov:Entity enthält physische, digitale, konzeptuelle oder andere Arten von Dingen mit bestimmten festen Aspekten. Eine prov:Activity ist eine Sache, die über einen bestimmten Zeitraum auftritt und auf oder mit einer prov: Entity wirkt. Sie kann das Konsumieren, Modifizieren, Verlagern, Verwenden oder Erzeugen der prov:Entity umfassen. Die Klasse prov:Agent ist die Klasse von Dingen, die in irgendeiner Form für eine stattfindende prov:Activity oder für die Existenz einer prov: Entity verantwortlich ist. ${ }^{64}$

Das PROV Alignment Module aus SSNO setzt einige Klassen und Properties von SOSA mit denen aus PROV-O in Beziehung. Die folgende Abbildung 6 veranschaulicht diese Beziehung:

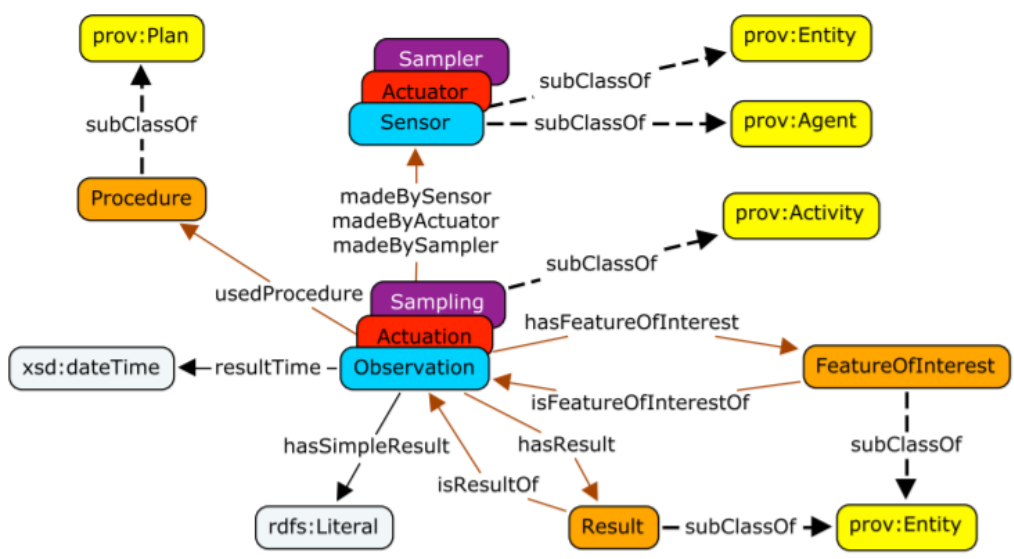

Abbildung 6: Beziehung zwischen SOSA und der PROV-Ontologie.

Quelle: Janowicz, K. / Haller, H. / Cox, S. / Phuoc, D. / Lefraņ̧ois, M. (2019): SOSA: A Lightweight Ontology for Sensors, Observations, Samples, and Actuators, S. 3, http://armin-haller.com/publications/SOSAJWS.pdf, Zugriff am 15.01.2020.

Abbildung 6 zeigt, dass sosa:Sensor, sosa:Actuator und sosa:Sampler sowohl Unterklassen von prov:Entity und prov:Agent sind. Sosa:Observation, sosa:Actuation und sosa:Sampling sind Unterklassen von prov:Activity. Mit Hilfe des Alignments ist es zum Beispiel möglich, einer sosa:Observation oder einer sosa:Actuation einen prov:Agent zuzuweisen, der für diese verantwortlich ist. Dasselbe gilt beispielsweise auch für einen sosa:Sensor oder einen sosa:Sampler. Ein prov:Agent kann auch Unterklassen haben wie prov:Person und prov:Organization. Diese werden häufig noch mit Hilfe der Friend of a Friend (FOAF) Ontologie genauer beschrieben, indem Namen oder Emailadressen zusätzlich als Literale angegeben werden können.

\subsubsection{Das Dataset Usage Vocabulary}

Das Dataset Usage Vocabulary (DUV) wurde von der Data on the Web Best Practices Working Group erstellt und ermöglicht es Nutzern von Datensätzen Erfahrungen, Zitate und Rückmeldungen über diese zu beschreiben und auszutauschen. ${ }^{65}$

Abbildung 7 gibt einen Überblick über den modularen Aufbau von DUV:

${ }^{64}$ Vgl. Provenance Working Group (2013): PROV-O: The PROV Ontology, https://www.w3.org/TR/2013/REC-prov-o-20130430/, Zugriff am 22.01.2020.

${ }^{65}$ Vgl. Data on the Web Working Group (2016): Data on the Web Best Practices: Dataset Usage Vocabulary, https://www.w3.org/TR/2016/NOTE-vocab-duv-20161215/, Zugriff am 23.01.2020. 
4. Evaluation der Semantic Sensor Network Ontology und der verbundenen Ontologien zur Wahl der Informationsquellen für die Facetten des GEO Labels
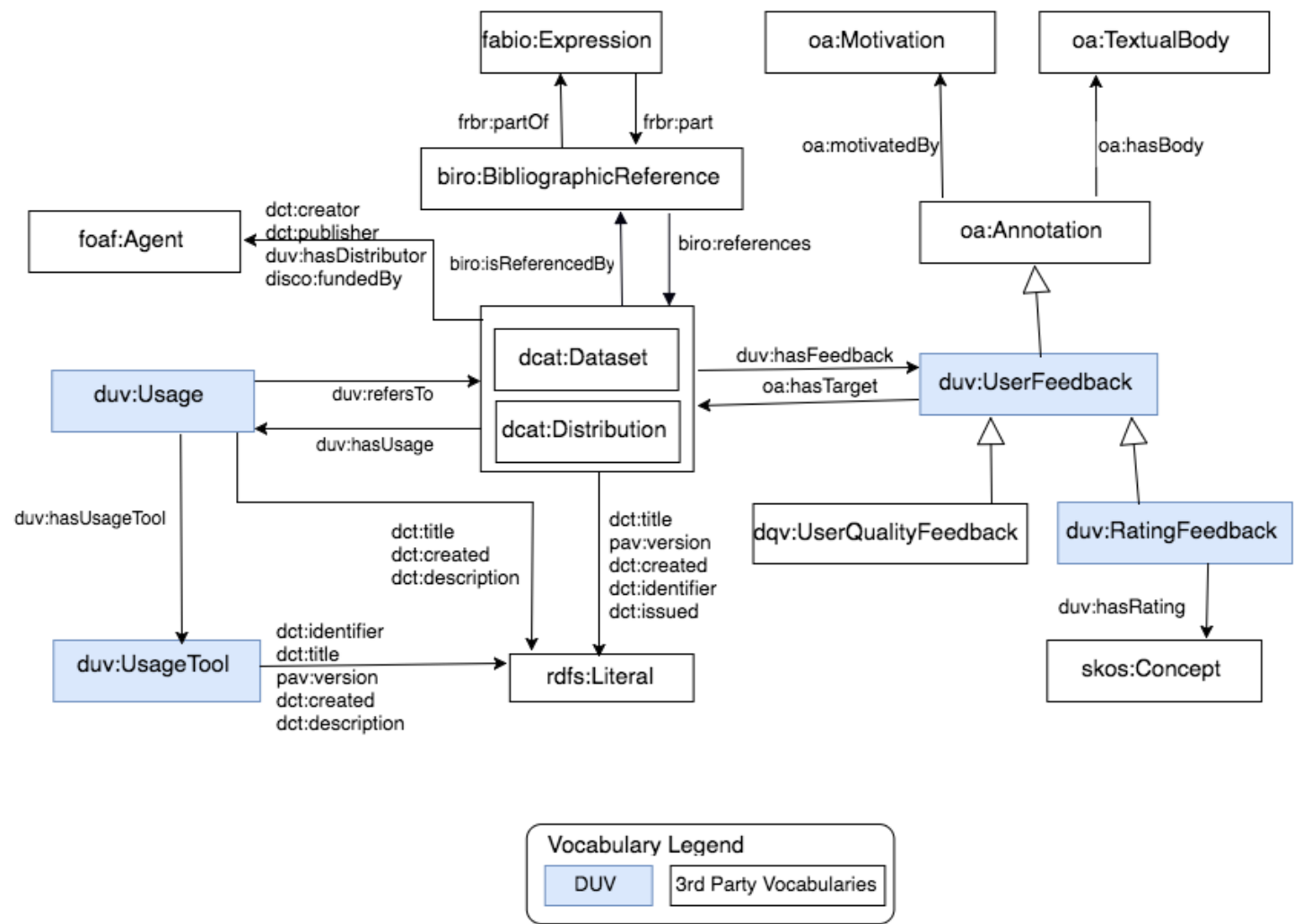

Abbildung 7: Überblick über den Aufbau des Dataset Usage Vocabulary.

Quelle: Data on the Web Working Group (2016): Data on the Web Best Practices: Dataset Usage Vocabulary, https://www.w3.org/TR/2016/NOTE-vocab-duv-20161215/, Zugriff am 23.01.2020.

In Abbildung 7 ist zu erkennen, dass das Vokabular viele Begriffe aus anderen Vokabularen verwendet. Ausgangspunkt ist immer ein dcat:Dataset oder eine dcat:Distribution. Diese Begriffe stammen aus dem Data Catalog Vocabulary (DCAT). Ein dcat: Dataset ist eine Sammlung von Daten, die veröffentlicht und in einer oder mehreren Darstellungen zum Zugriff oder Download zur Verfügung stehen. ${ }^{66}$ Um mit Hilfe von DUV SSNO-basierten RDF-Graphen Informationen über Nutzerfeedback oder Zitaten hinzuzufügen, wird die sosa:Observation, sosa:Actuation oder das sosa:Sampling an die Position von dcat:Dataset gestellt. An dieser Stelle soll kein vollständiges Ontology-Alignment erstellt werden, allerdings werden für diesen Zweck die genannten Klassen aus SOSA als Unterklassen von dcat:Dataset gesehen. So ist es möglich, diese mit einem duv:UserFeedback für die Facette User Feedback oder einer biro:BibliographicReference für die Facette Citation Information zu verknüpfen.

Für die Facette Expert Reviews kann DUV mit PROV-O verknüpft werden, indem man duv:UserFeedback als eine Unterklasse von prov: Entity ansieht. So kann ein User Feedback mit Hilfe von weiteren Klassen und Properties aus PROV-O als Feedback von einem Experten gekennzeichnet werden.

${ }^{66}$ Vgl. Dataset Exchange Working Group (2019): Data Catalog Vocabulary (DCAT) - Version 2, https://www.w3.org/TR/2019/PR-vocab-dcat-2-20191119/, Zugriff am 23.01.2020. 


\subsection{Wahl der Informationsquellen aus den evaluierten Ontologien für die Facetten des GEO Labels}

Nachfolgend wird die genaue Wahl der Klassen und Eigenschaften als Informationsquellen aus den evaluierten Ontologien erläutert, die notwendig sind, damit die jeweilige Facette des GEO Labels ausgefüllt wird. Dabei werden die Klassen und Eigenschaften, auf die in der Evaluation noch nicht eingegangen wurde, kurz erläutert.

Für die Hover-Over-Texte der Facetten werden Vorschläge zur Umsetzung gemacht, die Texte sollen innerhalb dieser Arbeit aber nicht mehr in die anschließende Implementierung integriert werden. Auch die Drilldown-Funktion, die strukturierte Informationen aus der als XML/RDFserialisierte Sensorbeschreibung anzeigen lassen würde, wird nicht implementiert.

\subsubsection{Producer Profile}

\section{Verfügbarkeit:}

Damit die Facette Producer Profile vollständig ausgefüllt wird, muss einer Klasse aus SSNO, die eine Unterklasse von prov:Activity oder prov:Entity ist, also beispielsweise ein sosa:Sensor oder eine sosa:Observation, ein prov:Agent zugewiesen werden. Für eine prov:Entity wird dafür die Property prov:wasAttributedTo verwendet, bei einer prov: Activity wird die Property prov:wasAssociatedWith eingesetzt, um die Verantwortung eines prov:Agent für eine prov:Activity zu kennzeichnen. Zudem muss noch eine Unterklasse von prov: Agent angegeben sein, also entweder prov: Person oder prov:Organization. Somit ist gewährleistet, dass der RDFGraph mindestens ein URI zu einer Person oder einer Organisation enthält, die die Verantwortung für eine der im Graphen beschriebenen prov:Activity oder prov: Entity trägt. Wenn diese Informationen in einem RDF-Graphen angegeben sind, wird die Facette Producer Profile ausgefüllt. ${ }^{67}$

\section{Hover-Over-Text:}

Im Hover-Over-Text könnte die Anzahl der angegeben Personen oder Organisationen aufgeführt werden. Da die URIs sehr lang sein könnten, könnte zusätzlich überprüft werden, ob beispielsweise der Name einer Organisation oder einer Person über die Properties foaf:name oder foaf: givenName im RDF-Graphen als Literal enthalten ist und diese gegebenenfalls statt der URIs anzeigen.

\subsubsection{Lineage Information}

\section{Verfügbarkeit:}

Damit die Facette Lineage Information ausgefüllt wird, sollte mindestens ein URI angegeben sein, der ein sosa:Procedure eindeutig kennzeichnet. Ein sosa: System ist beispielsweise über die Property ssn: implements mit einem sosa:Procedure in Beziehung gesetzt, das heißt, dass es eine Angabe über ein sosa:Procedure gibt, wenn die Property ssn:implements oder das dazugehörige

${ }^{67}$ Vgl. Provenance Working Group (2013). 
4. Evaluation der Semantic Sensor Network Ontology und der verbundenen Ontologien zur Wahl der Informationsquellen für die Facetten des GEO Labels

Inverse ssn:implementedBy im RDF-Graphen enthalten sind. Stattdessen könnte aber auch die Verfügbarkeit der Property sosa:usedProcedure überprüft werden, die einer Observation, Actuation oder einem Sampling eine sosa:Procedure zuweist. Zudem kann auch auf die Klasse sosa:Procedure selbst geprüft werden.

\section{Hover-Over-Text:}

Eine sosa:Procedure kann noch weiter beschrieben werden, zum Beispiel mit ssn: hasOutput und ssn: hasInput, wobei ssn:Output Informationen enthält, die als Bericht aus der sosa:Procedure hervorgehen und ssn:Input Informationen sind, die für die sosa:Procedure bereitgestellt werden. Für die Hover-Over-Texte könnte man die Anzahl der Inputs und Outputs eines sosa:Procedure angeben. ${ }^{68}$

\subsubsection{Producer Comments}

\section{Verfügbarkeit:}

Als Angabe von Herstellerkommentaren für die Facette Producer Comments wird, wie bereits erwähnt, rdfs: comment verwendet, da man hierbei davon ausgehen kann, dass die Kommentare von einer Person stammen, die an der Herstellung des Datensatzes mitgewirkt hat. Die Kommentare können dabei verschiedene Arten von Informationen beinhalten, nicht nur, wie in Tabelle 5 beschrieben, Informationen zur Qualität der Daten.

\section{Hover-Over-Text:}

Der Hover-Over-Text könnte die Anzahl der im RDF-Graphen enthaltenen rdfs:comment angeben.

\subsubsection{Compliance with Standards}

\section{Verfügbarkeit:}

Für diese Facette soll überprüft werden, ob es URIs gibt, die die Zeichenfolge w3.org enthalten. So kann festgestellt werden, ob Klassen und Properties im RDF-Graphen verwendet werden, die aus Ontologien und Vokabularen stammen, die von W3C, also einer offiziellen Stelle, als Empfehlung herausgegeben wurden.

\section{Hover-Over-Text:}

Als Hover-Over könnte ein Informationstext erscheinen, der angibt, dass unter anderem von W3C veröffentlichte Ontologien für die Beschreibung der Daten verwendet werden.

${ }^{68}$ Vgl. Spatial Data on the Web Working Group (2017). 


\subsubsection{Quality Information}

\section{Verfügbarkeit:}

Einem ssn:System kann eine (oder auch mehrere) ssn-system:SystemCapability zugewiesen werden. Eine ssn-system:SystemCapability ist eine Eigenschaft eines Systems für eine Messung unter einer bestimmten Bedingung (z.B. Temperatur) wie zum Beispiel ssn-system:Accuracy (Übereinstimmung zwischen dem Ergebnis einer Beobachtung und dem wahren Wert) oder ssn-system:Precision (Maß für die Fähigkeit eines Sensors, eine Beobachtung unter den definierten Bedingungen konsistent $z u$ reproduzieren). Ssn-system:Accuracy und ssn-system:Precision sind Unterklassen von ssn-system:SystemProperty. Sie werden über die Property ssn-system:hasSystemProperty mit der Klasse ssn-systems:SystemCapability verknüpft.

Zudem kann einem ssn:System eine ssn-system:OperatingRange übertragen werden. Diese beschreibt die normalen Betriebseigenschaften eines Systems unter bestimmten Bedingungen. Mit ssn-system:hasoperatingProperty wird der ssn-system:OperatingRange eine ssn-system:OperatingProperty zugewiesen, also ein identifizierbares Merkmal, das darstellt, wie das ssn: System unter angegeben Bedingungen arbeitet. Ssn-system:OperatingProperty hat die Unterklassen ssn-system:Maintenanceschedule (Wartungsplan für ein System) und ssn-system:OperatingPowerRange (Leistungsbereich, in dem das System unter den angegebenen Bedingungen betrieben werden soll).

Zuletzt kann einem ssn:System eine ssn-system:SurvivalRange zugewiesen werden, die die Lebensdauer eines ssn:Systems unter bestimmten Bedingungen beschreibt und mit ssn-system:hasSurvivalProperty eine ss-system:SurvivalProperty mit den Unterklassen ssn-system:SystemLifetime und ssn-system:BatteryLifetime übertragen bekommt.

Damit nun die Facette Quality Information ausgefüllt wird, sollte mindestens eine der drei Properties ssn-system:hassystemProperty, ssn-system:hasoperatingProperty oder ssn-system:hasSurvivalProperty im RDF-Graphen vorhanden sein. Zwar geben Unterklassen wie ssn-system:SystemLifetime und ssn-system:BatteryLifetime nicht direkt Informationen zur Qualität des Wertes einer Messung aus, dafür aber über das System selbst, das die Messung ausführt, weshalb diese Klassen trotzdem für diese Facette berücksichtig werden.

Es gibt noch eine weitere Property, mit der die Qualität einer sosa:Observation benutzerspezifischer angegeben werden kann: ssn-system:qualityofobservation. Mit dieser Property lässt sich noch einmal die Qualität einer sosa:Observation beurteilen. Ist diese vorhanden, wird die Facette Quality Information auch ausgefüllt. ${ }^{69}$

\section{Hover-Over-Text:}

Im Hover-Over-Text könnten neben der Anzahl der Informationen die Namen der oben beschriebenen Unterklassen angezeigt werden, damit der Nutzer sieht, welche Art von Qualitätsinformationen im RDF-Graphen enthalten sind.

${ }^{69} \mathrm{Vgl}$. Spatial Data on the Web Working Group (2017). 


\subsubsection{User Feedback}

\section{Verfügbarkeit:}

Eine Information über ein User Feedback liegt vor, wenn zum Beispiel eine sosa:Observation über die Property duv:hasUserFeedback mit einem duv:UserFeedback verknüpft ist. Statt einem duv:UserFeedback kann auch ein duv:RatingFeedback angegeben werden. Dieses beinhaltet vordefinierte Kriterien, um die Meinung eines Benutzers über einen Datensatz auszudrücken. ${ }^{70}$

\section{Hover-Over-Text:}

Zunächst könnte die Anzahl der vorhandenen Feedbacks aufgeteilt in duv:UserFeedback und duv:RatingFeedback angegeben werden. Falls Feedback-Kommentare als Literale im RDFGraphen verknüpft sind, könnte beispielhaft ein Kommentar angezeigt werden. Ein Kommentar kann mit oa:hasBody (Property aus der Web Annotation Ontology) an ein duv:UserFeedback angehängt werden.

\subsubsection{Expert Reviews}

\section{Verfügbarkeit:}

Damit die Facette Experts Reviews ausgefüllt wird, muss wie für die Facette User Feedback über die Property duv:hasUserFeedback ein duv:UserFeedback angegeben sein. Weiterhin wird duv:UserFeedback mit der Property prov:qualifiedAssociation eine prov:Association zugewiesen. Für das duv:UserFeedback wird also ein prov: Agent angegeben, der verantwortlich für das Feedback ist. Mit prov: hadRole kann dem prov: Agent eine Rolle hinzugefügt werden, die dieser bei der Erstellung des duv:UserFeedback hatte. Die Rollenbezeichnungen selbst sind nicht in PROV-O definiert. In diesem Fall sollte die Rolle die Zeichenkette expert enthalten. ${ }^{71}$

\section{Hover-Over-Text:}

Auch hier könnte die Anzahl der vorhandenen Feedbacks von Experten angegeben werden sowie, falls als Literal enthalten, ein Kommentar als Beispiel.

\subsubsection{Citations Information}

\section{Verfügbarkeit:}

Für die Facette Citations Information sollte die Property biro:isReferencedBy im RDF-Graphen enthalten sein, die eine Ressource, zum Beispiel eine sosa:Observation, mit einem die Ressource beschreibenden Literaturhinweis verbindet. ${ }^{72}$

\section{Hover-Over-Text:}

Im Hover-Over-Text kann die Anzahl der enthaltenen biro: ReferencedBy angegeben werden.

\footnotetext{
${ }^{70} \mathrm{Vgl}$. Data on the Web Working Group (2016).

${ }^{71}$ Vgl. Provenance Working Group (2013), Bsp. prov:hadRole: https://www.w3.org/TR/prov-o/\#Revision.

${ }^{72} \mathrm{Vgl}$. Shotton, D. \& Peroni, S. (2013): BiRO, the Bibliographic Reference Ontology, https://sparontologies.github.io/biro/current/biro.html, Zugriff am 26.01.2020.
} 


\section{Vergleich von Anbietern für Serverless Cloud-Computing und Wahl eines geeigneten Anbieters für die Bereitstellung der Java GEO Label API}

In diesem Kapitel sollen anhand von selbst definierten Kriterien Anbieter von Serverless CloudComputing verglichen und anschließend mindestens ein Anbieter zur Bereitstellung der Java GEO Label API gewählt werden.

\subsection{Definition der Vergleichskriterien}

Da die Basisimplementierung der GEO Label API auf Java basiert, müssen die Anbieter Java als Programmiersprache unterstützen. Zudem basiert der Code auf Java 8, weshalb diese Version entsprechend unterstützt werden muss.

In dieser Arbeit soll keine Kostenaufstellung durchgeführt werden, trotzdem bieten viele Anbieter ein kostenloses Kontingent für ihre Services an, was zur Durchführung dieser Arbeit von Vorteil ist.

Zuletzt müssen die Anbieter gegebenenfalls Integrationsmöglichkeiten mit anderen Services anbieten, damit der Code durch Events ausgeführt wird. In diesem Fall wäre ein Event ein POST oder GET Request. Es muss also Optionen geben, eine API auf Basis einer serverlosen Anwendung zu erstellen.

\subsection{Vergleich und Wahl des Anbieters}

Um die Auswahl der möglichen Anbieter einzuschränken, werden in der folgenden Tabelle 6 bereits nur Anbieter vergleichen, die Code in Java unterstützen.

Tabelle 6: Vergleich von Anbietern für Serverless Cloud-Computing.

Quelle: Eigene Darstellung.

\begin{tabular}{|l|l|l|l|}
\hline Anbieter & $\begin{array}{l}\text { Java } \\
\text { Version }\end{array}$ & $\begin{array}{l}\text { Kostenloses } \\
\text { Kontingent }\end{array}$ & Integrationsmöglichkeiten \\
\hline $\begin{array}{l}\text { Amazon Web } \\
\text { Services (AWS) }\end{array}$ & Java 8 & Ja & $\begin{array}{l}\text { AWS Lambda kann den Code (die Lambda- } \\
\text { Funktion) als Reaktion von HTTP- } \\
\text { Anforderungen über Amazon API Gateway } \\
\text { ausführen, einem Amazon Service zum Erstellen } \\
\text { und Überwachen von APIs. }\end{array}$ \\
\hline $\begin{array}{l}\text { Microsoft Azure } \\
\text { Functions }\end{array}$ & Java 8 & Ja & $\begin{array}{l}\text { Eine Azure Function kann über einen HTTP- } \\
\text { Trigger ausgelöst werden, wenn eine Anfrage } \\
\text { gestellt wird. Es gibt verschiedene Trigger, die } \\
\text { eine Azure Function auslösen können. }\end{array}$ \\
\hline
\end{tabular}

${ }^{73} \mathrm{Vgl.}$ Amazon Web Services (2020a): Funktionen von AWS Lambda, https://aws.amazon.com/de/lambda/features/, Zugriff am 29.01.2020.

74 Vgl. Microsoft Azure (2017): HTTP-Trigger und -Bindungen in Azure Functions, https://docs.microsoft.com/de-de/azure/azure-functions/functions-bindings-http-webhook?tabs=csharp, Zugriff am 29.01.2020. 
5. Vergleich von Anbietern für Serverless Cloud-Computing und Wahl eines geeigneten Anbieters für die Bereitstellung der Java GEO Label API

\begin{tabular}{|l|l|l|l|}
\hline \hline $\begin{array}{l}\text { IBM Cloud } \\
\text { Functions }\end{array}$ & Java 8 & Ja & $\begin{array}{l}\text { IBM Cloud Function kann zusammen mit IBM } \\
\text { Cloud API Gateway verwendet werden, ein IBM } \\
\text { Service zum Erstellen und Verwalten von APIs. }\end{array}$ \\
\hline $\begin{array}{l}\text { Google Cloud } \\
\text { Run }\end{array}$ & Java 8 & Ja & $\begin{array}{l}\text { Mit Google Cloud Run werden zustandslose } \\
\text { Container bereitgestellt, die beispielsweise } \\
\text { HTTP-Anfragen überwachen können. Dafür ist } \\
\text { kein weiterer Google Service nötig. }\end{array}$ \\
\hline
\end{tabular}

Aus Tabelle 6 geht hervor, dass alle vier verglichenen Anbieter Java 8 unterstützen sowie ein kostenloses Kontingent anbieten, sodass die Bereitstellung der GEO Label API in dieser Arbeit kostenlos getestet werden kann. Daher muss die Wahl des Anbieters anhand der Integrationsmöglichkeiten mit anderen Services getroffen werden. Alle Anbieter bieten Möglichkeiten zur Bereitstellung von APIs. Google Cloud Run wäre in dieser Arbeit deshalb eine gute Wahl, da die Implementierung des GEO Label Service bereits lokal in einem Tomcat-DockerContainer ausgeführt werden kann ${ }^{77}$. So müsste nur ein Dockerfile für die Erstellung eines Images erzeugt werden, sodass der Container, der die HTTP-Anfragen überwacht, bereitgestellt werden kann. Zudem würde der Container nicht nur die GEO Label API zur Verfügung stellen, sondern den gesamten GEO Label Service.

Neben Google Cloud Run soll die GEO Label API auch mit AWS Lambda bereitgestellt werden, um einen Vergleich zwischen beiden Anbietern herzustellen. Zudem ist AWS Lambda nicht Container-basiert, sondern nutzt den zusätzlichen Service Amazon API Gateway zum Aufrufen der Lambda-Funktion. Im Folgenden werden beide Anbieter noch einmal genauer beschrieben.

\section{Google Cloud Run}

Google Cloud Run gehört zur Google Cloud Platform (GCP). „,Die GCP besteht aus einer Reihe von physischen Ressourcen wie Computer und Festplattenlaufwerke sowie aus virtuellen Ressourcen wie virtuelle Maschinen (VMs), die über die Google-Rechenzentren weltweit zur Verfügung gestellt werden. ${ }^{\text {"78 }}$ Über bestimmte Dienste erhält der Nutzer Zugriff auf diese Ressourcen. Google Cloud bietet eine kostenlose Stufe. Diese enthält eine 12-monatige Testversion mit einem Guthaben von 300 \$. Zudem sind einige Ressourcen immer kostenlos. ${ }^{79}$

Cloud Run ist eine verwaltete Computing-Plattform, mit der zustandslose Container automatisch skaliert werden, das heißt, dass Cloud Run serverlos arbeitet und keine Infrastrukturverwaltung benötigt. Die Verwendung von Container ermöglicht die Nutzung beliebiger Programmiersprachen. ${ }^{80}$

\footnotetext{
${ }^{75} \mathrm{VgI.} \mathrm{IBM} \mathrm{(2020):} \mathrm{Serverunabhängige} \mathrm{REST-APIs} \mathrm{erstellen,}$ https://cloud.ibm.com/docs/openwhisk?topic=cloud-functions-apigateway, Zugriff am 29.01.2020.

${ }^{76} \mathrm{Vgl}$. Google (o.J.a): Cloud Run, https://cloud.google.com/run/?hl=de, Zugriff am 29.01.2020.

${ }^{77}$ Weiterführende Informationen verfügbar unter: https://github.com/anikagraupner/GEO-label-java/tree/master/server\#run-in-container.

${ }^{78}$ Google (o.J.b): Google Cloud Platform, https://cloud.google.com/docs/overview/?hl=de, Zugriff am 29.01.2020.

${ }^{79} \mathrm{Vgl.}$ Google (o.J.c): Kostenlose Stufe von Google Cloud, https://cloud.google.com/free/docs/gcp-free-tier?hl=de, Zugriff am 29.01.2020.

${ }^{80} \mathrm{Vgl}$. Google (o.J.a).
} 
5. Vergleich von Anbietern für Serverless Cloud-Computing und Wahl eines geeigneten Anbieters für die Bereitstellung der Java GEO Label API

Abbildung 8 zeigt das Cloud Run-Ressourcenmodell:

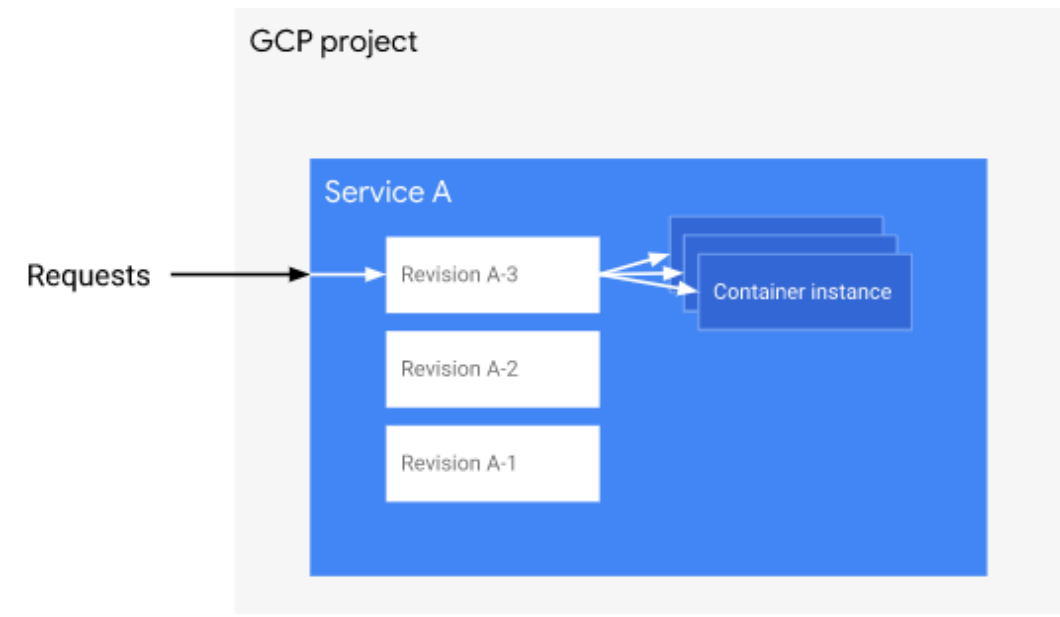

Abbildung 8: Cloud Run-Ressourcenmodell.

Quelle: Google (o.J.): Ressourcenmodell, https://cloud.google.com/run/docs/resource-model?hl=de, Zugriff am 30.01.2020.

In der Abbildung 8 ist ein GCP-Projekt erkennbar, das den Cloud Run-Dienst Service A bereitstellt. Der Dienst hat mehrere Revisionen (Überarbeitungen), wobei jede Revision ein bestimmtes Container-Image und Umgebungseinstellungen wie Arbeitsspeicherlimit und Gleichzeitigkeitswert umfasst. Wenn eine Revision Anfragen empfängt, wird diese automatisch auf die Anzahl der Containerinstanzen skaliert, die zur Verarbeitung aller Anfragen nötig sind. Eine Containerinstanz kann mehrere Anfragen gleichzeitig empfangen, die maximale Anzahl kann mit der Gleichzeitigkeitseinstellung festgelegt werden. ${ }^{81}$

Die Standardeinstellung für die Gleichzeitigkeit beträgt 80 Anfragen (allgemeiner maximaler Wert) gleichzeitig pro Containerinstanz. Wenn mehr Instanzen Anfragen verarbeiten, werden mehr CPU-Ressourcen und Arbeitsspeicher verwendet, da diese pro Containerinstanz gesetzt werden, was zu höheren Kosten führen könnte. ${ }^{82}$

Die Standardeinstellung für Arbeitsspeicher beträgt 256 Mebibyte, für die CPU 1. Mit der Gleichzeitigkeit eines Dienstes sollte auch das Speicherlimit erhöht werden, bei Verringerung der Gleichzeitigkeit kann auch das Speicherlimit verringert werden, um Kosten zu sparen. ${ }^{83}$

Für die Kosten werden nur die tatsächlich genutzten Ressourcen auf eine Zehntelsekunde genau berechnet. Dazu zählen Rechenleistung in vCPU-Sekunde, Arbeitsspeicher pro GB-Sekunde, Anfragen pro Million Anfragen und Netzwerk. ${ }^{84}$

Neben den genannten Umgebungseinstellungen können zudem auch noch Angaben über die Zeit, in der eine Antwort zurückgegeben werden muss, und über die maximale Anzahl von Containerinstanzen gemacht werden.

\footnotetext{
${ }^{81}$ Vgl. Google (o.J.d): Ressourcenmodell, https://cloud.google.com/run/docs/resource-model?hl=de, Zugriff am 30.01.2020.

82 Vgl. Google (o.J.e): Gleichzeitigkeit, https://cloud.google.com/run/docs/about-concurrency?hl=de, Zugriff am 30.01.2020.

${ }^{83} \mathrm{Vgl}$. Google (o.J.f): Speicherlimits konfigurieren, https://cloud.google.com/run/docs/configuring/memory-limits?hl=de, Zugriff am 30.01.2020. ${ }^{84} \mathrm{Vgl.}$ Google (o.J.a).
} 
5. Vergleich von Anbietern für Serverless Cloud-Computing und Wahl eines geeigneten Anbieters für die Bereitstellung der Java GEO Label API

\section{AWS Lambda und Amazon API Gateway}

AWS Lambda und Amazon API Gateway sind zwei Dienste der Cloud-Plattform Amazon Web Services ${ }^{85}$ (AWS).

AWS Lambda ist ein serverloser Datenverarbeitungsservice, der Code beim Eintreten bestimmter Ereignisse ausführt. Der ausgeführte Code wird Lambda-Funktion genannt und nur bei Bedarf aufgerufen. Dabei wird automatisch eine Skalierung zur Unterstützung der eingehenden Anforderungen durchgeführt, indem so viele Instanzen der Funktion gestartet werden wie benötigt. ${ }^{86}$ Wenn die Lambda-Funktion zum ersten Mal aufgerufen wird, wird eine Instanz der Funktion erstellt, die die Handler-Methode ausführt. Nachdem die Antwort zurückgegeben wurde, bleibt die Funktion aktiv und wartet auf die Verarbeitung weiterer Ereignisse. Wenn während der Verarbeitung eines Ereignisses ein weiteres Ereignis eintritt, wird eine weitere Instanz initialisiert und die Ereignisse gleichzeitig verarbeitet. ${ }^{87}$

Die Kosten setzen sich aus der Anzahl der Anforderungen und der Dauer, also der Zeit bis zur Ausführung des Codes gerundet auf 100 Millisekunden, zusammen. Der Preis ist abhängig von der zugewiesenen Arbeitsspeichergröße. Das kostenlose Kontingent umfasst 1 Millionen kostenlose Anforderungen pro Monat und 400000 GB-Sekunden Datenverarbeitungszeit pro Monat. Im Gegensatz zu Google Cloud Run basiert das AWS Lambda Ressourcenmodell darauf, nur die Größe des Arbeitsspeichers für die Funktion auszuwählen. Die Rechenleistung und andere Ressourcen wie CPU werden proportional erhöht. ${ }^{88}$

Zusätzlich lässt sich noch die reservierte Gleichzeitigkeit (Concurrency) einer Funktion einstellen. Die Gleichzeitigkeit selbst ist eine Angabe über die Anzahl der Instanzen aller LambdaFunktionen, die zu einem bestimmten Zeitpunkt Anforderungen bedienen. Diese unterliegt regionalen Begrenzungen, daher lässt sich für eine einzelne Funktion die reservierte Gleichzeitigkeit einstellen, die zudem die maximale Gleichzeitigkeit der Funktion angibt und nur von dieser verwendet werden kann. ${ }^{89}$

Um eine Lambda Funktion als Reaktion auf eine HTTP-Anfrage auszuführen, kann Amazon API Gateway verwendet werden. „Amazon API Gateway ist ein vollständig verwalteter Service, der das Erstellen, Veröffentlichen, Warten, Überwachen und Sichern von APIs für Entwickler in jeder beliebigen Größenordnung vereinfacht. ${ }^{\prime 90}$ Es können verschiedene Arten von APIs entwickelt werden, darunter auch REST-APIs (Representational State Transfer). Die Kosten werden pro Million API-Aufrufe berechnet ${ }^{91}$.

\footnotetext{
${ }^{85}$ Weiterführende Informationen verfügbar unter: https://aws.amazon.com/de/what-is-aws/.

${ }^{86} \mathrm{Vgl}$. Amazon Web Services (2020a).

${ }^{87} \mathrm{Vgl}$. Amazon Web Services (2020b): Managing Concurrency for a Lambda Function, https://docs.aws.amazon.com/lambda/latest/dg/configuration-concurrency.html, Zugriff am 06.02.2020.

${ }^{88} \mathrm{Vgl.}$. Amazon Web Services (2020c): AWS Lambda - Preise, https://aws.amazon.com/de/lambda/pricing/, Zugriff am 05.02.2020.

${ }^{89} \mathrm{Vgl}$. Amazon Web Services (2020b).

${ }^{90}$ Amazon Web Services (2020d): Amazon API Gateway, https://aws.amazon.com/de/api-gateway/, Zugriff am 05.02.2020.

${ }^{91} \mathrm{Vgl}$. Amazon Web Services (2020d).
} 
Um zu verhindern, dass die API von zu vielen Anfragen überlastet wird, werden die Anfragen mit Hilfe des Token-Bucket-Algorithmus ${ }^{92}$ gedrosselt (Throttling). Ein Token steht für eine Anfrage. Es wird ein Limit für eine stationäre Rate (Steady-State-Rate) und einen Burst (maximale BucketGröße) von Anforderungseinreichungen für alles APIs eines Kontos festgelegt. Wenn diese Limits überschritten werden, wird die Fehlernachricht 429 Too Many Requests gesendet und der Client kann die fehlgeschlagenen Anforderungen in Raten erneut einreichen. Standardmäßig begrenzt API Gateway die Steady-State-Anforderungsrate auf 10.000 Anfragen pro Sekunde. Es begrenzt den Burst (d.h. die maximale Bucket-Größe) auf 5000 Anfragen über alle APIs innerhalb eines AWS-Kontos. Beim API-Gateway entspricht die Burst-Grenze der maximalen Anzahl gleichzeitiger Anforderungseinreichungen, die das API-Gateway jederzeit erfüllen kann, ohne die zuvor genannte Fehlerantwort zurückzugeben. ${ }^{93}$

\section{Erweiterung der Java GEO Label API um die prototypische Generierung SSNO-basierter Label und Bereitstellung als Cloud- basierte serverless Anwendung}

Diese Kapitel enthält die Erstellung von Testdaten und die Erweiterung der Java GEO Label API, die in Kapitel 3.3 beschrieben wird. Der Code wird als freie quelloffene Software auf GitHub ${ }^{94}$ und auf Zenodo ${ }^{95}$ zur Verfügung gestellt. Anschließend wird die GEO Label API als Cloud-basierte serverless Anwendung mit Google Cloud Run und AWS Lambda bereitgestellt.

\subsection{Testdaten}

Zunächst müssen Testdaten erstellt werden, um die spätere Implementierung sowie die Bereitstellung der GEO Label API testen zu können. Die Testdaten müssen zudem als RDF/XMLDokumente abgespeichert werden, da, wie in Kapitel 3.3 beschrieben, in der Implementierung XML-basierte Metadatendokumente mit XPATH abgefragt werden. Es bietet sich an, für die Testdaten die Beispieldaten ${ }^{96}$ aus der Spezifikation von SSNO zu übernehmen und gegebenenfalls zu erweitern. Diese Daten liegen in Turtle vor, einer weiteren Serialisierung für RDF-Graphen, und müssen somit noch in RDF/XML konvertiert werden. Da es für einen RDFGraphen keine eindeutige RDF/XML-Serialisierung gibt, sondern mehrere Möglichkeiten zur Darstellung der Tripel (siehe Kapitel 2.1.2), soll bei der Erstellung der Testdaten darauf geachtet werden, dass mehrere RDF/XML-Serialisierungen für einen Graphen abgedeckt werden. Daher werden bei der Konvertierung von Turtle in RDF/XML zwei verschiedene Online-Konverter benutzt, der Easy RDF Converter ${ }^{97}$ (ERC) und der MyBluemix RDF Validator und Converter ${ }^{98}$ (MBC), die sich in ihren RDF/XML Darstellungen deutlich unterscheiden.

\footnotetext{
92 Weiterführende Informationen unter: https://de.wikipedia.org/wiki/Token-Bucket-Algorithmus.

${ }^{93}$ Vgl. Amazon Web Services (2020e): Throttle API Requests for Better Throughput,

https://docs.aws.amazon.com/apigateway/latest/developerguide/api-gateway-request-throttling.html,

Zugriff am 06.02.2020.

${ }^{94}$ Code verfügbar unter: https://github.com/anikagraupner/GEO-label-java.

${ }_{95}$ Code verfügbar unter: https://zenodo.org/record/3665628\#.XkREzGhKhPY.

${ }^{96}$ Beispieldaten verfügbar unter: https://www.w3.org/TR/vocab-ssn/\#examples.

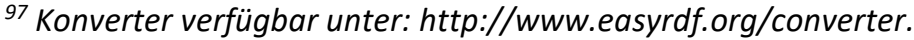

${ }^{98}$ Konverter verfügbar unter: http://rdfvalidator.mybluemix.net/.
} 
6. Erweiterung der Java GEO Label API um die prototypische Generierung SSNO-basierter Label und Bereitstellung als Cloud-basierte serverless Anwendung

Zunächst wird sichergestellt, dass für jede Facette eine Testdatei erstellt wird, die diese Facette bei der Label Generierung abdeckt. Zudem wird eine Datei erstellt, bei der alle Facetten nach der Generierung des Labels als verfügbar gekennzeichnet werden. Alle Datensätze werden im Ordner testdata ${ }^{99}$ abgelegt.

Im Anhang 3 befindet sich ein RDF-Graph serialisiert in RDF/XML, der Informationen zu allen Facetten enthält und mit dem Easy RDF Converter von Turtle nach RDF/XML konvertiert wurde. Im Anhang 4 ist derselbe RDF-Graph auch als RDF/XML-Serialisierung abgelegt, dieser wurde allerdings mit dem MyBluemix Validator and Converter von Turtle nach RDF/XML konvertiert.

\subsection{Erweiterung der Java GEO Label API}

Um die Implementierung um die Generierung SSNO-basierter Label zu erweitern, muss eine neue Transformationsdatei hinzugefügt werden. Der Inhalt der Datei wird unter Kapitel 6.2.1 beschrieben und erläutert. Zusätzlich müssen zum Test der Generierung der Labels einige Unittests erstellt werden. Letztlich soll dem Nutzer ein Image vom GEO Label Service auf Docker Hub zur lokalen Generierung von Labels zur Verfügung gestellt werden.

\subsubsection{Transformationsdatei für SSNO-basierte Metadaten}

In der Datei server.properties ${ }^{100}$ (Anhang 5) wird zunächst der Pfad zur transformerSSNO.json ${ }^{101}$ angegeben, damit diese Datei neben den bereits bestehenden Transformationsdateien als Transformationsressource verfügbar ist:

http://geoviqua.github.io/geolabel/mappings/transformerSSNO.json=/transformations/tra nsformerSSNO.json

Dabei wird auch eine URL aufgeführt, für den Fall, dass die Transformationsdatei für SSNO auch online gestellt werden soll, damit von verschiedenen Anwendungen aus darauf zugegriffen werden kann. Aktuell ist die Datei aber nur lokal verfügbar, weshalb auch der lokale Pfad zur transformerSSNO.json angegeben wird.

Im Folgenden wird der Aufbau der transformerSSNO.json beschrieben. Die Datei ist auch im Anhang 6 aufgeführt.

\section{Applicability Path (applicabilityPath):}

Der Applicability Path ist ein XPATH-Ausdruck, mit dem überprüft wird, welche Transformationsdatei für das Metadatendokument, aus dem ein Label generiert werden soll, geeignet ist. In der transformerSSNO.java lautet der Pfad:

boolean $\left(/{ }^{*}\left[\right.\right.$ local - name ()$=$ RDF $\left.\left.^{\prime}\right]\right)$

\footnotetext{
${ }^{99}$ Ordner verfügbar unter: https://github.com/anikagraupner/GEO-label-java/tree/master/testdata.

${ }^{100}$ Datei verfügbar unter: https://github.com/anikagraupner/GEO-label-

java/blob/master/server/src/main/resources/server.properties.

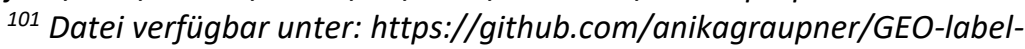

java/blob/master/server/src/main/resources/transformations/transformerSSNO.json.
} 
6. Erweiterung der Java GEO Label API um die prototypische Generierung SSNO-basierter Label und Bereitstellung als Cloud-basierte serverless Anwendung

Mit diesem wird abgefragt, ob der erste Knoten bzw. Wurzelknoten im Metadatendokument mit RDF bezeichnet ist. Dies trifft nur auf die RDF/XML-Dokumente zu, die mit dieser Arbeit für die Labelgenerierung eingeführt werden. Falls die Implementierung nochmals um Metadatendokumente erweitert wird, die auf semantischen Technologien, aber nicht unbedingt auf SSNO basieren, sollte der Applicability Path erneut angepasst werden.

\section{Namespace Mappings (namespaceMappings):}

Die Namespace Mappings vereinfachen die in der JSON-Datei angegebenen XPATH-Ausdrücke, da in diesen nicht mehr local-name() verwendet werden muss. Stattdessen können in den XPATH-Ausdrücken die Präfixe verwendet werden, die für diese Arbeit in Tabelle 6 festgelegt wurden. Diese Präfixe werden mit den zugehörigen Namensräumen in der Transformationsdatei unter den Namespace Mappings angegeben. Falls in einem Metadatendokument nun andere Präfixe für dieselben Namensräume verwendet, werden die Elemente bzw. Knoten im XPATHAusdruck trotzdem richtig erkannt.

\section{Facet Descriptions (facetDescriptions):}

Unter den Facet Descriptions wird für jede Facette der Availability Path (availabilityPath) angegeben. Mit diesem wird überprüft, ob Informationen für diese Facette im Metadatendokument verfügbar sind oder nicht, sodass die Facette entweder ausgefüllt wird oder nicht. Zudem wird für jede Facette das String-Template für den Hover-Over-Text angegeben sowie die XPATH-Ausdrücke, die Informationen in das Template einfügen. Zuletzt ist jeweils eine URL für die Drilldown-Funktion aufgeführt.

Die Hover-Over-Texte sollen in dieser Arbeit in der Transformationsdatei für SSNO-basierte Metadaten nicht umgesetzt werden, deshalb wird für jede Facette folgendes festgelegt:

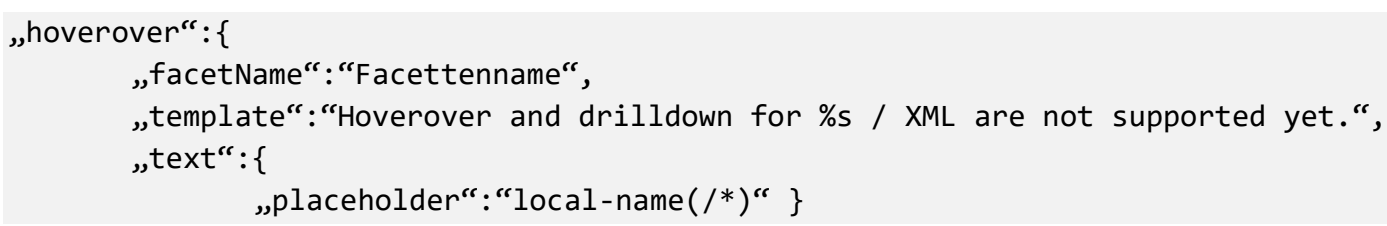

Damit wird für jede Facette im generierten Label der Hover-Over-Text Hoverover and drilldown for RDF / RML are not supported angezeigt. Es ist notwendig, den XPATH-Ausdruck für den placeholder einzufügen, damit der Code richtig funktioniert. Allerdings werden hin und wieder die falschen Hover-Over-Texte für SSNO-basierte Labels angezeigt. Dies ist aber ein Problem in der Basisimplementierung und ist nicht auf die transformerSSNO.java zurückzuführen. Für die Drilldown-Funktion sind URLs angegeben. Wie bereits erwähnt wird diese Funktion aber nicht umgesetzt.

Der XPATH-Ausdruck für den Availability Path wird nun für jede Facette einzeln erläutert. Dabei wird für jeden XPATH-Ausdruck die Funktion boolean verwendet, um zu testen, ob der in der Funktion enthaltene Ausdruck wahr oder falsch ist. Wenn der Ausdruck wahr ist, wird die jeweilige Facette ausgefüllt, trifft der Ausdruck nicht zu, wird die Facette nicht ausgefüllt. 
6. Erweiterung der Java GEO Label API um die prototypische Generierung SSNO-basierter Label und Bereitstellung als Cloud-basierte serverless Anwendung

\section{Producer Profile:}

(boolean(//*[rdf:Description[@rdf:about=//prov:wasAssociatedWith/@rdf:resource or @rd $f:$ about=//prov:wasAttributedTo/@rdf:resource]/rdf:type[@rdf:resource='http://www.w3.o rg/ns/prov\#Organization' or @rdf:resource='http://www.w3.org/ns/prov\#Person']])) or ( boolean(//*[prov:wasAttributedTo/prov:Agent/rdf:type[@rdf:resource='http://www.w3.org /ns/prov\#Person' or @rdf: resource='http://www.w3.org/ns/prov\#Organization']])) or (boolean(//*[prov:wasAssociatedWith/prov:Agent/rdf:type[@rdf:resource='http://www. w3.org/ns/prov\#Person' or @rdf: resource='http://www.w3.org/ns/prov\#Organization']])) or (boolean(//*[prov:Agent[@rdf:about=//prov:wasAssociatedWith/@rdf:resource or @rdf: about=//prov:wasAttributedTo/@rdf:resource]/rdf:type[@rdf:resource='http://www.w3.org /ns/prov\#Organization' or @rdf:resource='http://www.w3.org/ns/prov\#Person']]))

Mit dem Ausdruck wird abgefragt, ob die unter Kapitel 4.2.1 beschriebenen notwendigen Datenfelder in einem Metadatendokument enthalten sind, damit die Facette Producer Profile als verfügbar gekennzeichnet wird. An der Wiederholung der abgefragten Klassen und Properties in diesem Ausdruck ist gut zu erkennen, dass die Möglichkeiten der unterschiedlichen RDF/XML-Serialisierungen dazu führen, dass der Ausdruck sehr komplex wird. Die folgenden beiden Ausschnitte aus den Testdaten (oben: MyBluemix Converter, unten: Easy RDF Converter) veranschaulichen diese RDF/XML-Serialisierungen:

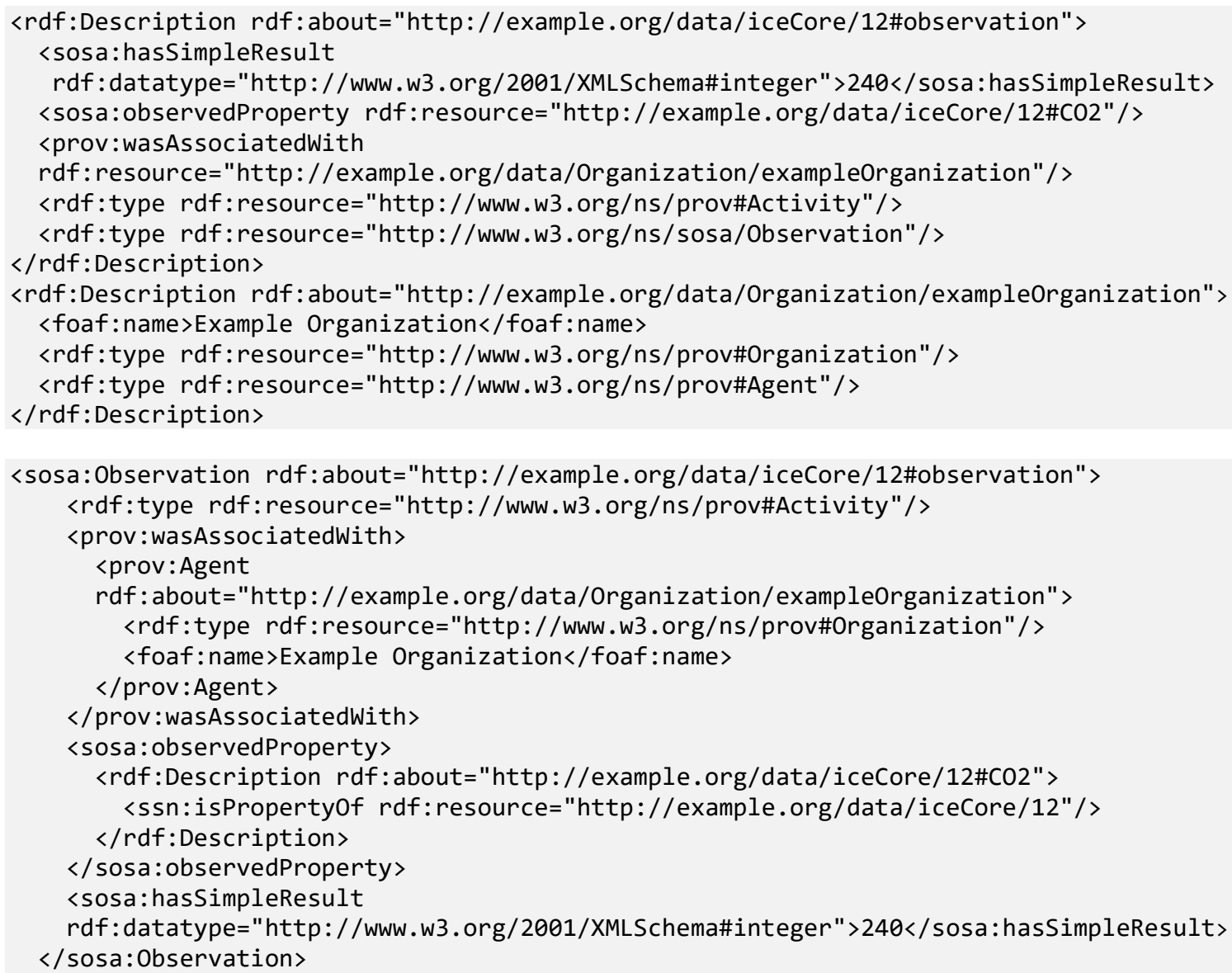

In beiden Ausschnitten sind dieselben Informationen zur Facette Producer Profile enthalten, allerdings kann nicht derselbe XPATH-Ausdruck verwendet werden, um abzufragen, ob beispielsweise eine prov: Organization angegeben ist, da sich die Strukturen in den Ausschnitten unterscheiden. 
6. Erweiterung der Java GEO Label API um die prototypische Generierung SSNO-basierter Label und Bereitstellung als Cloud-basierte serverless Anwendung

\section{Lineage Information:}

boolean(//*[ssn:implements or ssn:implementedBy or sosa:usedProcedure or sosa:Procedu re or @rdf:resource='http://www.w3.org/ns/sosa/Procedure'])

Mit diesem XPATH-Ausdruck wird überprüft, ob die unter Kapitel 4.2.2 genannten Informationen für die Facette Lineage Information im Dokument enthalten sind.

\section{Producer Comments:}

boolean $\left(/ /^{*}[\right.$ rdfs:comment $\left.]\right)$

Der XPATH-Ausdruck prüft auf rdfs: comment Elemente im RDF/XML-Dokument für die Facette Producer Comments (siehe Kapitel 4.2.3).

\section{Compliance with Standards:}

boolean (//*[contains(@*, 'w3.org')])

Wie in Kapitel 4.2.4 beschrieben werden die URIs von allen Attributbezeichnungen mit Hilfe des XPATH-Ausdrucks auf die Zeichenkette w3.org geprüft. Eine Prüfung der Namensräume auf w3.org wäre noch effektiver, allerdings lässt sich dies mit XPATH 1.0 nicht umsetzen.

\section{Quality Information:}

boolean(//*[ssn-system:hasSystemProperty or ssn-system:hasoperatingProperty or ssnsystem:hasSurvivalProperty or ssn-system:qualityofobservation])

Der XPATH-Ausdruck prüft auf die in Kapitel 4.2.5 genannten Datenfelder für die Facette Quality Information.

\section{User Feedback:}

(boolean(//*[duv:hasFeedback/duv:UserFeedback[not(prov:qualifiedAssociation)] or duv: hasFeedback/duv:RatingFeedback])) or (boolean(//*[rdf:Description[@rdf:about=//duv: ha sFeedback/@rdf:resource]/rdf:type[@rdf:resource='http://www.w3.org/ns/duv\#UserFeedbac $k^{\prime}$ or @rdf:resource='http://www.w3.org/ns/duv\#RatingFeedback']]) and boolean $(/ / *[r d f$ : Description[@rdf:about=//duv:hasFeedback/@rdf:resource][not(prov:qualifiedAssociation )] ])) or (boolean(//*[duv:UserFeedback[@rdf:about=//duv:hasFeedback/@rdf:resource][no $\mathrm{t}$ (prov:qualifiedAssociation)]])) or (boolean(//*[duv:RatingFeedback[@rdf:about=//duv: hasFeedback/@rdf:resource]]))

Mit diesem XPATH-Ausdruck wird nach einem User Feedback im RDF/XML-Dokument gesucht (siehe Kapitel 4.2.6). Die Ausdrücke [not(prov:qualifiedAssociation)] sind notwendig, um zu verhindern, dass die Facette User Feedback ausgefüllt wird, obwohl das Feedback als Expert Review gekennzeichnet ist.

\section{Expert Reviews:}

(boolean(//*[duv:hasFeedback/duv:UserFeedback/prov:qualifiedAssociation/prov: Associat ion/prov:hadRole/prov:Role[contains(@rdf:about, 'expert')]])) or (boolean(//*[rdf:Des cription[@rdf:nodeID=//rdf:Description[@rdf:about=//duv:hasFeedback/@rdf:resource]/pr ov:qualifiedAssociation/@rdf:nodeID]/prov:hadRole[contains(@rdf:resource, 'expert')] ] 
6. Erweiterung der Java GEO Label API um die prototypische Generierung SSNO-basierter Label und Bereitstellung als Cloud-basierte serverless Anwendung

)) or (boolean(//*[duv:UserFeedback[@rdf:about=//duv:hasFeedback/@rdf:resource]/prov: qualifiedAssociation/prov:Association/prov:hadRole/prov:Role[contains(@rdf:about, 'ex pert')]]))

Im Gegensatz zum XPATH-Ausdruck für die Facette User Feedback wird für die Facette Expert Reviews geprüft, ob es das Element prov:qualifiedAssociation gibt sowie weitere der unter Kapitel 4.2.7 genannten Datenfelder. Mit [contains(@rdf: about, 'expert' )] wird abgefragt, ob die Bezeichnung der prov:Role bzw. der URI der Ressource die Zeichenkette expert enthält.

\section{Citations Information:}

boolean $(/ / *[$ biro:isReferencedBy $])$

Der XPATH-Ausdruck prüft nur auf das Vorhandensein des angegebenen Elements, das in Kapitel 4.2.8 erläutert wird, für die Facette Citations Information.

\subsubsection{Unittests}

Für die Unittests wird eine neue Testdatei im server-Modul hinzugefügt, die MetadataTransformerTest_SSNO.java ${ }^{102}$. In dieser werden mit Hilfe von JUnit einige Tests zur Generierung von SSNO-basierten Labels entworfen. Für jede Facette des Labels wird ein Test erstellt, der sicherstellt, dass bei der Generierung des Labels aus den jeweiligen Testdaten die entsprechende Facette als verfügbar gekennzeichnet wird. Dabei werden die RDF/XMLSerialisierungen aus beiden Konvertern berücksichtigt. Zudem wird ein Test verfasst, bei dem eine Testdatendatei verwendet wird, bei der alle Facetten ausgefüllt werden sollen. Weiterhin wird getestet, ob die Generierung des Labels auch erfolgreich ist, wenn in einer Testdatei andere Präfixe für die Namensräume angegeben sind, als in den XPATH-Ausdrücken der Transformationsdatei für SSNO. Mit dem Test wird also geprüft, ob die Namespace Mappings in der transformerSSNO.json funktionieren. Zusätzlich werden noch zwei Tests erstellt, bei denen das generierte Label direkt als SVG lokal beim Nutzer gespeichert wird. Unter Anhang 7 befinden sich einige Test aus der MetadataTransformerTest_SSNO.java. Die gesamte Testklasse kann erfolgreich ausgeführt werden (Anhang 8).

\subsubsection{Java GEO Label API mit Docker Image lokal ausführen}

Damit der Nutzer lokal auf seinem Rechner ein Label aus einem Metadatensatz generieren kann, wird ein Image auf Docker Hub zur Verfügung gestellt. Zunächst wird ein Dockerfile ${ }^{103}$ erstellt, der die folgenden Befehle enthält:

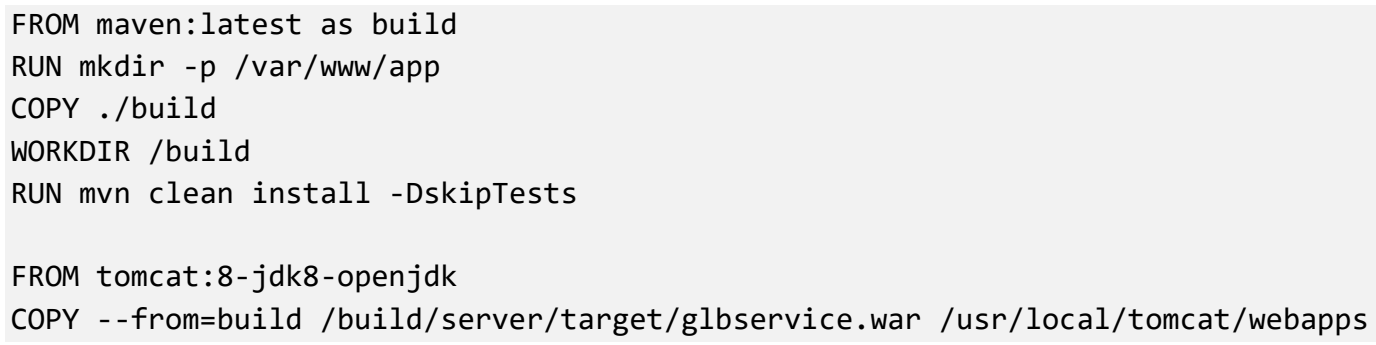

102 Datei verfügbar unter: https://github.com/anikagraupner/GEO-label-

java/blob/master/server/src/test/java/org/n52/geolabel/server/mapping/MetadataTransformerTest_SS NO.java.

${ }^{103}$ Date verfügbar unter: https://github.com/anikagraupner/GEO-label-java/blob/master/Dockerfile. 
6. Erweiterung der Java GEO Label API um die prototypische Generierung SSNO-basierter Label und Bereitstellung als Cloud-basierte serverless Anwendung

Copy --from=build/build/misc/tomcat-users.xml /usr/local/tomcat/conf

EXPOSE 8080

CMD [,catalina.sh","run"]

Im ersten Teil wird das Basisimage (maven) festgelegt, ein neues Verzeichnis erstellt, in das die Dateien kopiert werden sowie ein Arbeitsverzeichnis festgelegt, in dem mvn clean install DskipTests ausgeführt wird. Der zweite Teil sorgt dafür, dass der GEO Label Service mit der GEO Label API in einem Tomcat-Docker-Container läuft.

Mit Hilfe des Dockerfiles wird ein Image auf Docker Hub öffentlich zur Verfügung gestellt. Dieses kann mit den folgenden Docker-Befehlen lokal ausgeführt werden:

docker pull ani18/geolabel

docker run -p 8080:8080 ani18/geolabel

Für einen GET Request kann beispielsweise die folgende URL in einem Browser geöffnet werden:

http://localhost:8080/glbservice/api/v1/svg?metadata=https://raw.githubusercontent.co m/anikagraupner/GEO-label-

java/master/testdata/All_Facets_Available/RDFXML/ERC_EasyRDFConverter/ERC_all_factes_ available_ip68smartsensor_unknown_prefixes.rdf

Die angegebene URL für den Parameter metadata führt zu der Testdatei, in der Informationen für alle Facetten enthalten sind. Abbildung 9 veranschaulicht das im Browser angezeigte Label:

$\leftarrow \rightarrow$ C (i) localhost:8080/glbservice/api/v1/svg?metadata=https://ri

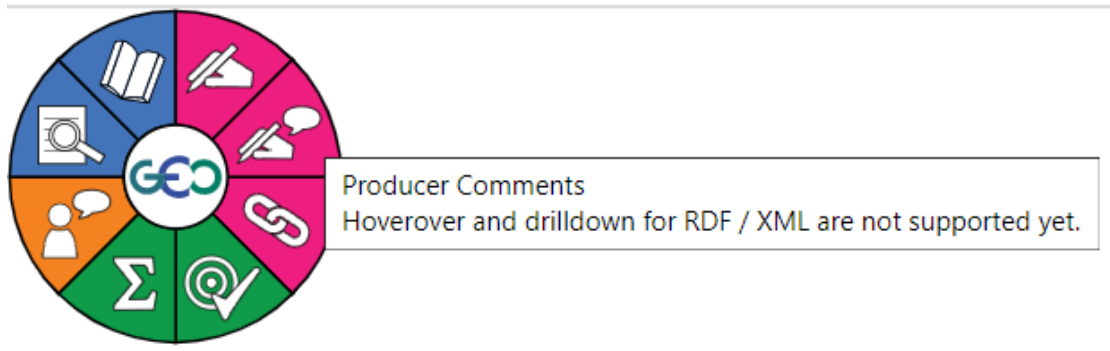

Abbildung 9: GEO Label als Scalable Network Graphic, lokal generiert.

Quelle: Eigene Darstellung.

In der Abbildung 9 wird auch der Hover-Over-Text angezeigt, der angibt, dass für RDF/XMLDokumente noch keine Hover-Over-Texte und Drilldown-Funktionen unterstützt werden.

Alternativ kann ein Nutzer auch wie folgt einen POST Request mit Curl ${ }^{104}$ an den Server schicken:

curl -X POST -F "metadata=@filename.rdf" http://localhost:8080/glbservice/api/v1/svg -output label.svg

Mit dem Befehl --output wird ein Pfad und ein Dateiname mit der Endung .svg angegeben, um das generierte Label direkt in einer lokalen Datei zu abzuspeichern.

${ }^{104}$ Weiterführende Informationen verfügbar unter: https://curl.haxx.se/. 


\subsection{Bereitstellung der Java GEO Label API}

Im Folgenden wird beschrieben, wie die GEO Label API mit Google Cloud Run und AWS Lambda (bzw. Amazon API Gateway) bereitgestellt wird. Zudem werden die Cloud-basierten Labelgenerierungen mit einer Test-Suite getestet, indem auch einige Nutzerszenarien erstellt werden, anhand derer die Performanz bewertet und gegebenenfalls verbessert werden soll.

\subsubsection{Bereitstellung mit Google Cloud Run}

Für die Bereitstellung der GEO Label API (bzw. des gesamten GEO Label Service) muss zunächst ein neues Projekt auf der Google Cloud Platform erstellt werden, beispielsweise mit dem Namen geolabel-java-api. Dieses muss als Arbeitsbereich ausgewählt werden. Anschließend wird die Google Cloud Run API aktiviert und die Cloud Shell ${ }^{105}$ geöffnet, eine Befehlszeile, mit der direkt vom Browser aus auf die Cloud-Ressourcen zugegriffen werden kann. Die folgenden Befehle können nacheinander in der Cloud Shell ausgeführt werden:

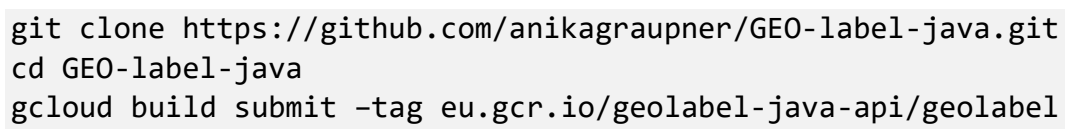

Mit Hilfe dieser Befehle wird der Code der GEO Label Service Implementierung von GitHub geklont. Nachdem man in das Verzeichnis gewechselt ist, das den Dockerfile aus Kapitel 6.2.3 enthält, wird das Container Image erstellt. Dieses ist nun in der Container Registry ${ }^{106}$ verfügbar, einem zentralen Ort, an dem die Docker-Images verwaltet werden.

Das Image könnte nun mit einem weiteren Befehl in der Cloud Shell mit Cloud Run bereitgestellt werden. An dieser Stelle wird aber der Button Dienst erstellen auf der Cloud Run Seite verwendet. Als Container-Image-URL wird die URL vom zuvor erstellten Image aus der Container Registry verwendet:

eu.gcr.io/geolabel-java-api/geolabel (, eu' gibt die Region an)

Zudem muss eine Region ausgewählt werden, in der sich die Infrastruktur befindet, die den Cloud Run Dienst ausführt (z.B. europe-west1). Als Dienstname wird glbservice festgelegt. Weiterhin muss für die Authentifizierung die Option Nicht authentifizierte Aufrufe zulassen angeklickt werden, damit die API für alle zugänglich ist.

Darüber hinaus werden für die in Kapitel 5.2 beschriebenen Umgebungseinstellungen die Standardeinstellungen übernommen (Arbeitsspeicher $=256$ Mebibyte, $C P U=1$, Gleichzeitigkeit $=80$ ). Ob diese Einstellungen ausreichen, wird anschließend mit Apache JMeter getestet. Gegebenenfalls müssen neue Revisionen mit anderen Einstellungen erstellt werden.

In Abbildung 10 sieht man den mit Google Cloud Run bereitgestellten Dienst bzw. die bereitgestellte Revision für die GEO Label API mit den gewählten Einstellungen:

${ }^{105}$ Weiterführende Informationen verfügbar unter: https://cloud.google.com/shell/?hl=de.

${ }^{106}$ Weiterführende Informationen verfügbar unter: https://cloud.google.com/container-registry/?hl=de. 
6. Erweiterung der Java GEO Label API um die prototypische Generierung SSNO-basierter Label und Bereitstellung als Cloud-basierte serverless Anwendung

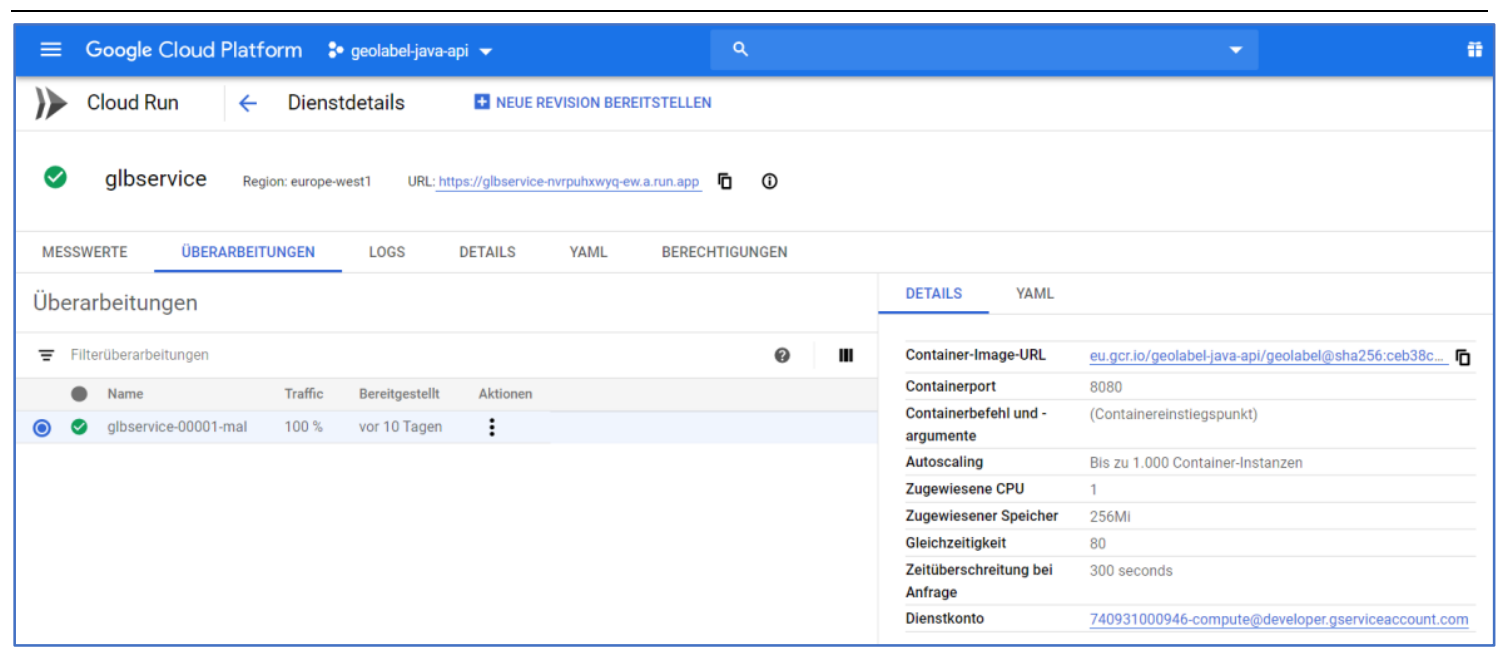

Abbildung 10: Google Cloud Run Dienst zur Bereitstellung der GEO Label API, aktuell mit einer Revision. Quelle: Eigene Darstellung.

Die mit Google Cloud Run bereitgestellte GEO Label API ist nun verfügbar unter:

https://glbservice-nvrpuhxwyq-ew.a.run.app/glbservice/api/v1

Es können alle in Kapitel 3.3 beschriebenen Endpunkte und Parameter zur Labelgenerierung verwendet werden. In Abbildung 11 ist ein Label als PNG abgebildet, dass über die Google Cloud Run API generiert wurde und auf einer Sensorbeschreibung basiert, die Informationen zu den Facetten Producer Comments und Standards Compliance enthält:

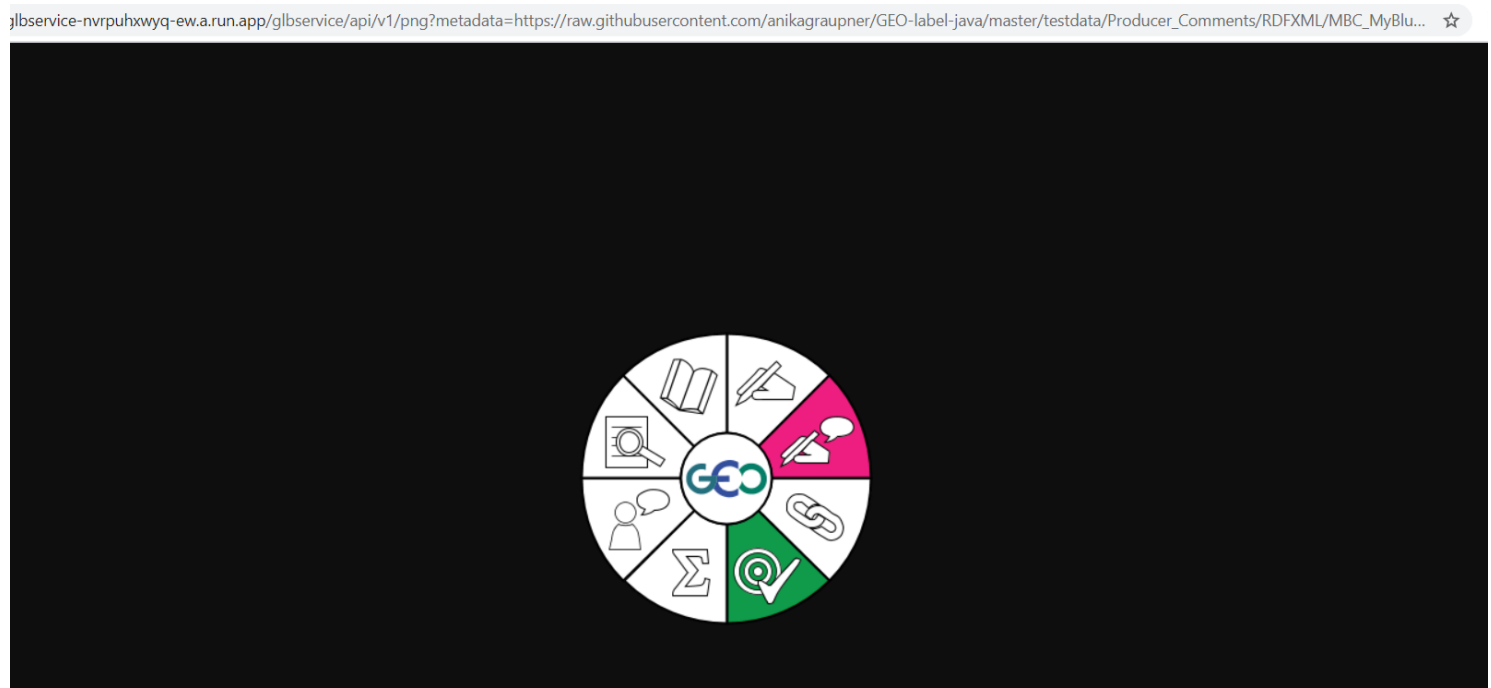

Abbildung 11: GEO Label als Portable Network Graphic, generiert über die mit Google Cloud Run bereitgestellte GEO Label API.

Quelle: Eigene Darstellung.

Weiterhin ist auch die Beispielseite unter folgender URL zu erreichen:

https://glbservice-nvrpuhxwyq-ew.a.run.app/glbservice

Auch die Bearbeitungsseite mit dem Textfeld ist verfügbar:

https://glbservice-nvrpuhxwyq-ew.a.run.app/glbservice/editor.jsf 
6. Erweiterung der Java GEO Label API um die prototypische Generierung SSNO-basierter Label und Bereitstellung als Cloud-basierte serverless Anwendung

Damit ein Label generiert werden kann, muss zunächst über den Button Add Service folgender API-Endpunkt hinzugefügt werden:

https://glbservice-nvrpuhxwyq-ew.a.run.app/glbservice/api/v1/svg

Anschließend lassen sich Label aus dem Inhalt des Textfeldes generieren.

\subsubsection{Bereitstellung mit AWS Lambda und Amazon API Gateway}

Für die Bereitstellung der API mit AWS Lambda und Amazon API Gateway wird dem MavenProjekt ein neues Modul namens lambda hinzugefügt, das um die Klasse APIHandler.java ${ }^{107}$ erweitert wird. Diese Klasse implementiert den Request-Handler, der die Parameter einer Anfrage von API Gateway empfängt und verarbeitet. Die gesamte Datei ist im Anhang 9 verfügbar.

Die Handler-Methode handleRequest verwendet InputStream und OutputStream als Eingabeund Ausgabetypen. Die Parameter der Anfrage an API Gateway werden als JSON an die HandlerMethode weitergegeben. Die Bytes des InputStream werden deshalb mit einem InputStreamReader und einem BufferedReader in Zeichen decodiert. Anschließend wird ein JSON-Objekt aus dem Input generiert. Das JSON Objekt enthält die Pfadparameter der Anfrage wie api/v1/svg sowie die Abfrageparameter wie metadata und feedback. Daher müssen je nach Angabe von Pfadparameter und Abfrageparameter die Antworten generiert werden.

Für die Generierung der Labels soll nur der Endpunkt zur Generierung eines Labels als SVG unter Angabe einer Metadaten-URL und/oder Feedback-URL unterstützt werden. Die anderen Endpunkte sollen in dieser Arbeit für AWS Lambda nicht umgesetzt werden.

Damit aus den eingegangenen Daten der Handler-Methode ein Label generiert wird, müssen aus der Handler-Methode andere Methoden der GEO Label Service Implementierung aufgerufen werden. Wenn der Abfrageparameter metadata und/oder der Abfrageparameter feedback jeweils mit URL angegeben sind und die Pfadparameter api/v1/svg vorhanden sind, wird die Methode updateGeoLabel aufgerufen, um aus dem Inhalt der URL das zuvor deklarierte Label zu aktualisieren. Anschließend wird mit der Methode toSVG eine temporäre Datei auf Basis eines SVG-Templates geschrieben, die das Label als SVG enthält. Der Inhalt der Datei wird wiederum ausgelesen und als Body der Antwort festgesetzt. Die Antwort einer Anfrage wird zunächst als JSON-Objekt erstellt. Dabei müssen Status-Code und Content-Type als Headers angegeben werden sowie der Body der Antwort. Mit einem Status Code wird angegeben, ob eine Anfrage erfolgreich bearbeitet wurde (200) oder nicht (400, 404, 500 etc.), der Content-Type klassifiziert die Daten. Zuletzt wird mit Hilfe eines OutpuStreamWriter das JSON-Objekt in Form eines OutputStreams als Antwort zurückgegeben.

Insgesamt werden noch weitere Pfadangaben abgefangen, beispielsweise wird bei der Angabe von api/v1 eine Information über die verfügbaren Endpunkte der API mit Content-Type application/json zurückgegeben. Für die Generierung des Labels ist aber wie bereits erwähnt nur die SVG-Generierung möglich.

${ }^{107}$ Datei verfügbar unter: https://github.com/anikagraupner/GEO-labeljava/blob/master/lambda/src/main/java/org/n52/geolabel/lambda/APIHandler.java. 
6. Erweiterung der Java GEO Label API um die prototypische Generierung SSNO-basierter Label und Bereitstellung als Cloud-basierte serverless Anwendung

Um nun eine Lambda-Funktion zu erstellen, müssen zunächst der Funktionsname (glbservice), die Programmiersprache (Java 8) und die Rolle, die die Berechtigungen für die Funktion innehat, festgelegt werden. Anschließen kann mit dem Befehl mvn clean package eine JAR-Datei für das Modul lambda erstellt werden und diese als Funktionscode hochgeladen werden. Der Handler gibt den Ort der Handler-Methode an. Die Standardeinstellung für den Arbeitsspeicher beträgt 512 Mebibyte. Da diese Lambda-Funktion bisher die einzige aktive Funktion für den verwendeten AWS Account ist, wird keine reservierte Gleichzeitigkeit festgelegt. Die regional begrenzte Gleichzeitigkeit (Europa Frankfurt) beträgt 1000.

Zuletzt wird mit Amazon API Gateway eine REST API erstellt. Als Name wird glbservice vergeben. Als Resource wird eine Proxy Resource erstellt mit Pfad \{proxy +$\}$. Zudem wird API Gateway CORS aktiviert. Anschließend wird eine ANY-Methode erstellt. Diese fängt alle Anfragen ab, unabhängig von der Angabe, ob es sich um eine GET, POST oder um eine andere Anfrage handelt. Als Integrationstyp wird Lambda Function gewählt, um anzugeben, dass eine LambdaFunktion auf die API-Anfragen reagiert. Zudem wird der Name der Lambda-Funktion festgelegt, also glbservice. Abbildung 12 zeigt die bereitgestellte API:

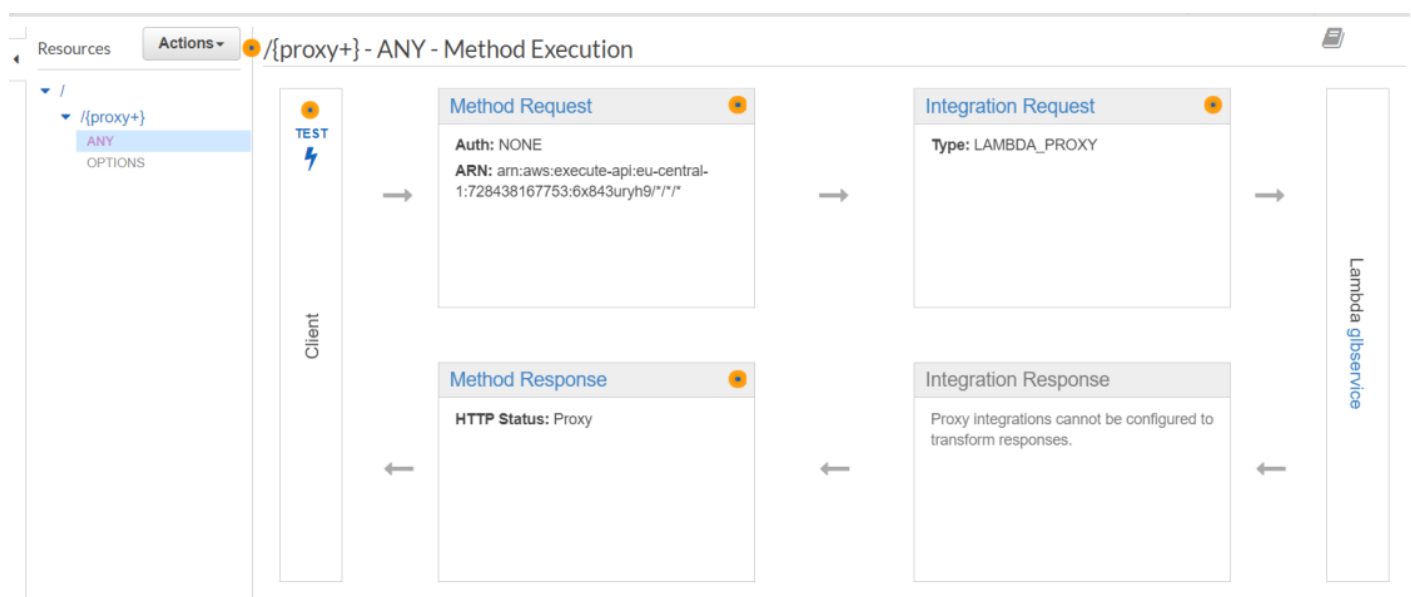

Abbildung 12: Mit Amazon API Gateway bereitgestellte API.

Quelle: Eigene Darstellung.

Wenn eine von einem Client gesendete Anfrage empfangen wird, wird die Lambda-Funktion ausgeführt und die in der Funktion generierte Antwort an den Client zurückgegeben. Um die API nun öffentlich zur Verfügung zu stellen, muss eine sogenannte Stage ${ }^{108}$ erstellt werden. Als Stage-Name wird glbservice angegeben. Zudem werden die Standardeinstellungen für die Drosselung (Throttling) beibehalten (Steady-State-Rate: 10000, Burst 5000). Die mit AWS Lambda und Amazon API Gateway bereitgestellte GEO Label API ist nun verfügbar unter:

https://6x843uryh9. execute-api.eu-central-1.amazonaws.com/glbservice/api/v1

Die Labelgenerierung als Scalable Vector Graphic kann unter folgendem Endpunkt angefragt werden:

https://6x843uryh9.execute-api.eu-central-1.amazonaws.com/glbservice/api/v1/svg

108 Weiterführende Informationen unter:

https://docs.aws.amazon.com/apigateway/latest/developerguide/set-up-stages.html. 
6. Erweiterung der Java GEO Label API um die prototypische Generierung SSNO-basierter Label und Bereitstellung als Cloud-basierte serverless Anwendung

Abbildung 13 veranschaulicht ein generiertes Label mit einer festgelegten Größe (size) von 500 und basierend auf einer Sensorbeschreibung, die Informationen zu den Facetten Producer Comments, Lineage Information, Standards Compliance und Quality Information enthält:

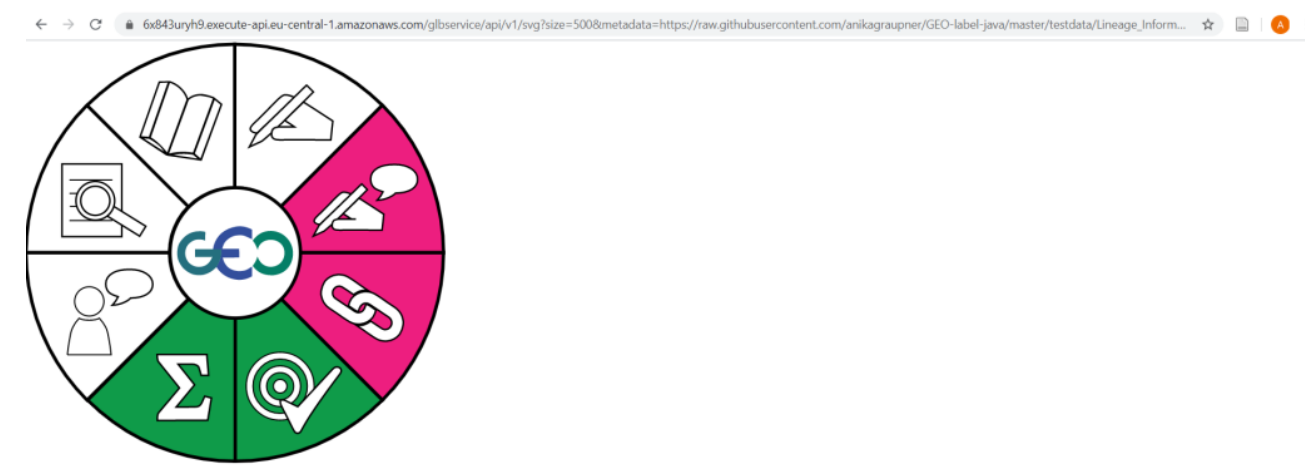

Abbildung 13: GEO Label als Scalable Vector Graphic, generiert über die mit AWS Lambda und API Gateway bereitgestellte GEO Label API.

Quelle: Eigene Darstellung.

\subsubsection{Performanz-Tests mit Apache JMeter}

Für das Testen der bereitgestellten GEO Label APIs wird Apache JMeter ${ }^{109}$ verwendet, eine Java Open-Source-Software zum Testen von Webanwendungen. JMeter ermöglicht es, zum Testen die Anzahl der Nutzer, die Anfragen senden, sowie die Zeit, die zwischen den einzelnen Anfragen liegt, anzugeben. Weiterhin kann festgelegt werden, wie oft die Anfragen wiederholt werden sollen. Der JMeter Test Plan (GEO_Label_API.jmx) und die hier vorgestellten tabellarischen Testergebnisse werden öffentlich auf GitHub zur Verfügung gestellt. ${ }^{110}$ Die Dateinamen werden im Folgenden in Klammern angegeben. Für alle API Anfragen wird für den Parameter metadata die Datei MBC_allfactes_available_ip68smartsensor.rdf ${ }^{111}$ über eine URL oder als Datei angegeben. Der Parameter feedback wird nicht angegeben.

Zunächst werden für die mit Google Cloud Run und AWS Lambda bereitgestellten GEO Label APIs einige allgemeine Tests erstellt, um die richtigen Funktionsweisen der APIs zu überprüfen. Dabei wird jeweils ein Nutzer angegeben, der einmal alle erstellten Anfragen ausführt. Für Google Cloud Run wird nicht nur die GEO Label API überprüft, sondern der gesamte GEO Label Service. Dabei wird kontrolliert, ob auch die Beispielseite (...glbservice) und die Bearbeitungsseite (...glbservice/editor.jsf) mit Status Code 200 und dem Content-Type text/html aufgerufen werden können. Weiterhin werden zur Generierung eines Labels für jeden Endpunkt der API jeweils ein GET und ein POST Request an die API gesendet, die mit Status Code 200 und dem korrekten Content-Type (image/svg+xml, image/png, application/xml) beantwortet werden müssen, damit der Test erfolgreich ist. Zudem wird geprüft, ob für die Endpunkte ...api und ...api/v1 Antworten mit Content-Type application/json zurückgegeben werden. Abbildung 14 zeigt das Ergebnis (GCR_General_Tests):

109 Weiterführende Informationen verfügbar unter: http://jmeter.apache.org/.

${ }^{110}$ Dateien verfügbar unter: https://github.com/anikagraupner/GEO-label-

java/tree/master/misc/JMeterTests, Anleitung zum Ausführen des Test Plan verfügbar unter: https://github.com/anikagraupner/GEO-label-java/blob/master/README.md\#jmeter-test-plan.

${ }_{111}$ Datei verfügbar unter: https://github.com/anikagraupner/GEO-

label-java/blob/master/testdata/All_Facets_Available/RDFXML/MBC_MyBluemixConverter/MBC_all_fa ctes_available_ip68smartsensor.rdf. 
6. Erweiterung der Java GEO Label API um die prototypische Generierung SSNO-basierter Label und Bereitstellung als Cloud-basierte serverless Anwendung

\begin{tabular}{|c|c|c|c|c|c|c|}
\hline Sample \# & Start Time & Sample Time(ms) & Status & Bytes & Sent Bytes & Latency \\
\hline & 19:38:28.374 GET Examplepage & 340 & 0 & 10908 & 293 & 231 \\
\hline & 19:38:28.715 GET Editorpage & 84 & $(0)$ & 7864 & 157 & 83 \\
\hline & 19:38:28.800 GET API Info & 39 & $(2)$ & 807 & 150 & 39 \\
\hline & 19:38:28.840 GET API Version Info & 51 & $(0)$ & 807 & 150 & 51 \\
\hline & 19:38:28.892 GET SVG Label & 246 & 0 & 22925 & 341 & 234 \\
\hline & 19:38:29.139 GET PNG Label & 462 & 0 & 35788 & 341 & 448 \\
\hline & 19:38:29.602 GET LML Label & 207 & 0 & 3721 & 341 & 207 \\
\hline & 19:38:29.810 POST SVG Label & 302 & (2) & 22629 & 16583 & 299 \\
\hline & 19:38:30.113 POST PNG Label & 511 & 8 & 35788 & 16571 & 486 \\
\hline & 19:38:30.625 POST LML Label & 552 & (2) & 3494 & 16571 & 552 \\
\hline
\end{tabular}

Abbildung 14: Tabellarisches Ergebnis allgemeine Tests Google Cloud Run API.

Quelle: Eigene Darstellung.

In Abbildung 14 ist zu erkennen, dass alle Anfragen einen grünen Status haben, das heißt, dass alle Anfragen hinsichtlich Status Code und Content-Type erfolgreich beantwortet wurden.

Für AWS Lambda können weniger Anfragen gestellt werden. Hierbei werden nur die Tests für die Anfragen zur Generierung eines Labels als SVG mit einem GET Request übernommen. Zudem wird auch hier geprüft, ob für die Endpunkte ...api und ...api/v1 Antworten mit Content-Type application/json zurückgegeben werden. Abbildung 15 veranschaulicht das Ergebnis (AWS_General_Tests):

\begin{tabular}{|c|c|c|c|c|c|c|c|}
\hline Sample \# & Start Time & Label & Sample Time(ms) & Status & Bytes & Sent Bytes & Latency \\
\hline 1 & $20: 09: 34.561$ & GET SVG Label & 1578 & 0 & 21619 & 356 & 1578 \\
\hline 2 & 20:09:36.141 & GET API Info & 62 & 0 & 471 & 165 & 62 \\
\hline 3 & $20: 09: 36.204$ & GET API Version Info & 30 & 6 & 471 & 165 & 30 \\
\hline
\end{tabular}

Abbildung 15: Tabellarisches Ergebnis allgemeine Tests AWS Lambda API.

Quelle: Eigene Darstellung.

Auch hier sind alle Tests erfolgreich.

Anhand von verschiedenen Nutzerszenarien wird nun überprüft, wie gut die Performanz der bereitgestellten APIs ist und ob die eingestellten Cloud-Ressourcen (Arbeitsspeicher, CPU, Gleichzeitigkeit) ausreichend sind. Bei beiden Anbietern wird mit den jeweils zuvor beschriebenen Standardeinstellungen für die Cloud-Ressourcen begonnen. Neben Google Cloud Run und AWS Lambda wird auch die Performanz der lokal in einem Tomcat-Docker-Container laufenden GEO Label API getestet. Der Container hat ca. 2 Gibibyte zur Verfügung. Da die mit AWS Lambda bereitgestellte API nur die Generierung von Labels als SVG unterstützt, werden in den Szenarios nur GET Requests mit Angabe der URL zur Testdatei als metadata Parameter verwendet. Zudem wird für jedes Szenario grundsätzlich überprüft, ob die einzelnen Antworten den Status Code 200 und den Content-Type image/svg+xml haben, um zu prüfen, ob gültige Antworten zurückgegeben werden.

\section{Szenario 1 - Auf einer Wetterdatenseite für die Stadt Münster suchen Nutzer nach geeigneten Wetterdaten, die mit Sensoren aufgenommen wurden, indem sie GEO Labels aus den Sensorbeschreibungen generieren.}

Für das Szenario werden zehn Nutzer angegeben, die jeweils einen GET Request an die API stellen. Zwischen den Anfragen liegen jeweils zwei Minuten. Die Antwortzeit einer Anfrage sowie der Mittelwert aller Antwortzeiten sollte höchstens zwischen 0,1 und 1 Sekunde bzw. zwischen 100 und 1000 Millisekunden liegen, da nach Jakob Nielsen 0,1 Sekunde die Grenze 
6. Erweiterung der Java GEO Label API um die prototypische Generierung SSNO-basierter Label und Bereitstellung als Cloud-basierte serverless Anwendung

dafür ist, dass der Nutzer das Gefühl hat, dass das System augenblicklich funktioniert und 1 Sekunde die Grenze dafür ist, dass der Gedankenfluss des Benutzers nicht unterbrochen wird. ${ }^{112}$

\section{Ergebnis Google Cloud Run (GCR Scenario 1 V1):}

\begin{tabular}{|c|c|c|c|c|c|c|c|}
\hline Sample \# & Start Time & Label & Sample Time(ms) & Status & Bytes & Sent Bytes & Latency \\
\hline 1 & $20: 46: 28.570$ & HTTP GET Request & 692 & 0 & 22929 & 341 & 630 \\
\hline 2 & $20: 48: 28.571$ & HTTP GET Request & 434 & (6) & 22929 & 341 & 374 \\
\hline 3 & $20: 50: 28.573$ & HTTP GET Request & 492 & 6 & 22929 & 341 & 420 \\
\hline 4 & $20: 52: 28.569$ & HTTP GET Request & 584 & (6) & 22929 & 341 & 517 \\
\hline 5 & $20: 54: 28.573$ & HTTP GET Request & 416 & 0 & 22929 & 341 & 369 \\
\hline 6 & $20: 56: 28.570$ & HTTP GET Request & 446 & 0 & 22929 & 341 & 385 \\
\hline 7 & $20: 58: 28.571$ & HTTP GET Request & 604 & (6) & 22929 & 341 & 577 \\
\hline 8 & 21:00:28.570 & HTTP GET Request & 435 & 6 & 22929 & 341 & 397 \\
\hline 9 & 21:02:28.569 & HTTP GET Request & 843 & 0 & 22929 & 341 & 816 \\
\hline 10 & $21: 04: 28.570$ & HTTP GET Request & 854 & 6 & 22929 & 341 & 786 \\
\hline
\end{tabular}

Abbildung 16: Tabellarisches Ergebnis Szenario 1 Google Cloud Run API.

Quelle: Eigene Darstellung.

Abbildung 16 zeigt, dass alle Anfragen einen grünen Status haben, das heißt, dass alle Antworten den Status Code 200, den Content-Type image/svg+xml und die Anfragen eine Antwortzeit (Sample Time) von unter 1000 Millisekunden haben. Der Mittelwert liegt bei 580 Millisekunden. Die eingestellten Cloud-Ressourcen sind also ausreichend.

\section{Ergebnis AWS Lambda (AWS Scenario 1 V1):}

\begin{tabular}{|c|c|c|c|c|c|c|c|}
\hline Sample \# & Start Time & Label & Sample Time(ms) & Status & Bytes & Sent Bytes & Latency \\
\hline 1 & $21: 20: 52.318$ & HTTP GET Request & 1028 & (x) & 21619 & 356 & 1028 \\
\hline 2 & 21:22:52.319 & HTTP GET Request & 978 & (2) & 21619 & 356 & 977 \\
\hline 3 & $21: 24: 52.321$ & HTTP GET Request & 715 & (2) & 21619 & 356 & 715 \\
\hline 4 & $21: 26: 52.318$ & HTTP GET Request & 914 & 0 & 21619 & 356 & 914 \\
\hline 5 & 21:28:52.319 & HTTP GET Request & 803 & (2) & 21619 & 356 & 803 \\
\hline 6 & $21: 30: 52.319$ & HTTP GET Request & 821 & 8 & 21619 & 356 & 821 \\
\hline 7 & 21:32:52.319 & HTTP GET Request & 1406 & (ख) & 21619 & 356 & 1405 \\
\hline 8 & 21:34:52.316 & HTTP GET Request & 776 & (8) & 21619 & 356 & 776 \\
\hline 9 & 21:36:52.317 & HTTP GET Request & 862 & (6) & 21619 & 356 & 862 \\
\hline 10 & 21:38:52.316 & HTTP GET Request & 657 & (2) & 21619 & 356 & 657 \\
\hline
\end{tabular}

Abbildung 17: Tabellarisches Ergebnis Szenario 1 AWS Lambda API.

Quelle: Eigene Darstellung.

In Abbildung 17 ist zu erkennen, dass acht von zehn Anfragen einen grünen Status haben, die Antworten also den Status Code 200, den Content-Type image/svg+xml und die Anfragen eine Antwortzeit (Sample Time) von unter 1000 Millisekunden haben. Zwei Antwortzeiten liegen knapp über 1000 Millisekunden, insgesamt liegt der Mittelwert aller Antwortzeiten aber bei 896 Millisekunden, weshalb die Cloud-Ressourcen als ausreichend eingestuft werden.

\section{Ergebnis Local Host (LH Scenario 1 V1):}

Der Mittelwert aller Antwortzeiten liegt bei 420 Millisekunden. Wie in Abbildung 18 veranschaulicht, werden alle Anfragen in unter 1000 Millisekunden gültig beantwortet:

112 Vgl. Nielsen, J. (1993): Usability Engineering, Kapitel 5,

https://www.nngroup.com/articles/response-times-3-important-limits/, Zugriff am 30.01.2020. 
6. Erweiterung der Java GEO Label API um die prototypische Generierung SSNO-basierter Label und Bereitstellung als Cloud-basierte serverless Anwendung

\begin{tabular}{|c|c|c|c|c|c|c|c|}
\hline Sample \# & Start Time & Label & Sample Time(ms) & Status & Bytes & Sent Bytes & Latency \\
\hline 1 & $21: 55: 15.118$ & HTTP GET Request & \begin{tabular}{|r}
337 \\
\end{tabular} & 0 & 22811 & 321 & 337 \\
\hline 2 & $21: 57: 15.118$ & HTTP GET Request & 367 & (2) & 22811 & 321 & 366 \\
\hline 3 & 21:59:15.121 & HTTP GET Request & 561 & 8 & 22811 & 321 & 560 \\
\hline 4 & 22:01:15.120 & HTTP GET Request & 298 & (2) & 22811 & 321 & 296 \\
\hline 5 & 22:03:15.121 & HTTP GET Request & 439 & 0 & 22811 & 321 & 438 \\
\hline 6 & $22: 05: 15.120$ & HTTP GET Request & 509 & 0 & 22811 & 321 & 508 \\
\hline 7 & 22:07:15.123 & HTTP GET Request & 345 & (2) & 22811 & 321 & 344 \\
\hline 8 & 22:09:15.120 & HTTP GET Request & 461 & 0 & 22811 & 321 & 460 \\
\hline 9 & $22: 11: 15.120$ & HTTP GET Request & 534 & (0) & 22811 & 321 & 532 \\
\hline 10 & $22: 13: 15.123$ & HTTP GET Request & 349 & 0 & 22811 & 321 & 348 \\
\hline
\end{tabular}

Abbildung 18: Tabellarisches Ergebnis Szenario 1 Local Host API.

Quelle: Eigene Darstellung.

Szenario 2 - Auf einer Wetterdatenseite für ganz Europa suchen Nutzer nach geeigneten Wetterdaten, die mit Sensoren aufgenommen wurden, indem sie GEO Labels aus den Sensorbeschreibungen generieren.

Für das Szenario werden 1000 Nutzer definiert, die in Abständen von 1 Sekunde jeweils einen GET Requests an die API schicken. So wird simuliert, dass kontinuierlich Anfragen verarbeitet werden. Auch hier sollte die Antwortzeit einer Antwort sowie der Mittelwert aller Antwortzeiten höchstens zwischen 0,1 und 1 Sekunde bzw. zwischen 100 und 1000 Millisekunden liegen. Die Ergebnisse werden mit Graphen veranschaulicht, die Standardabweichung, Mittelwert und Median in Millisekunden über den gesamten Anfragezeitraum hinweg abbilden.

Ergebnis Google Cloud Run (GCR Scenario 2 V1):

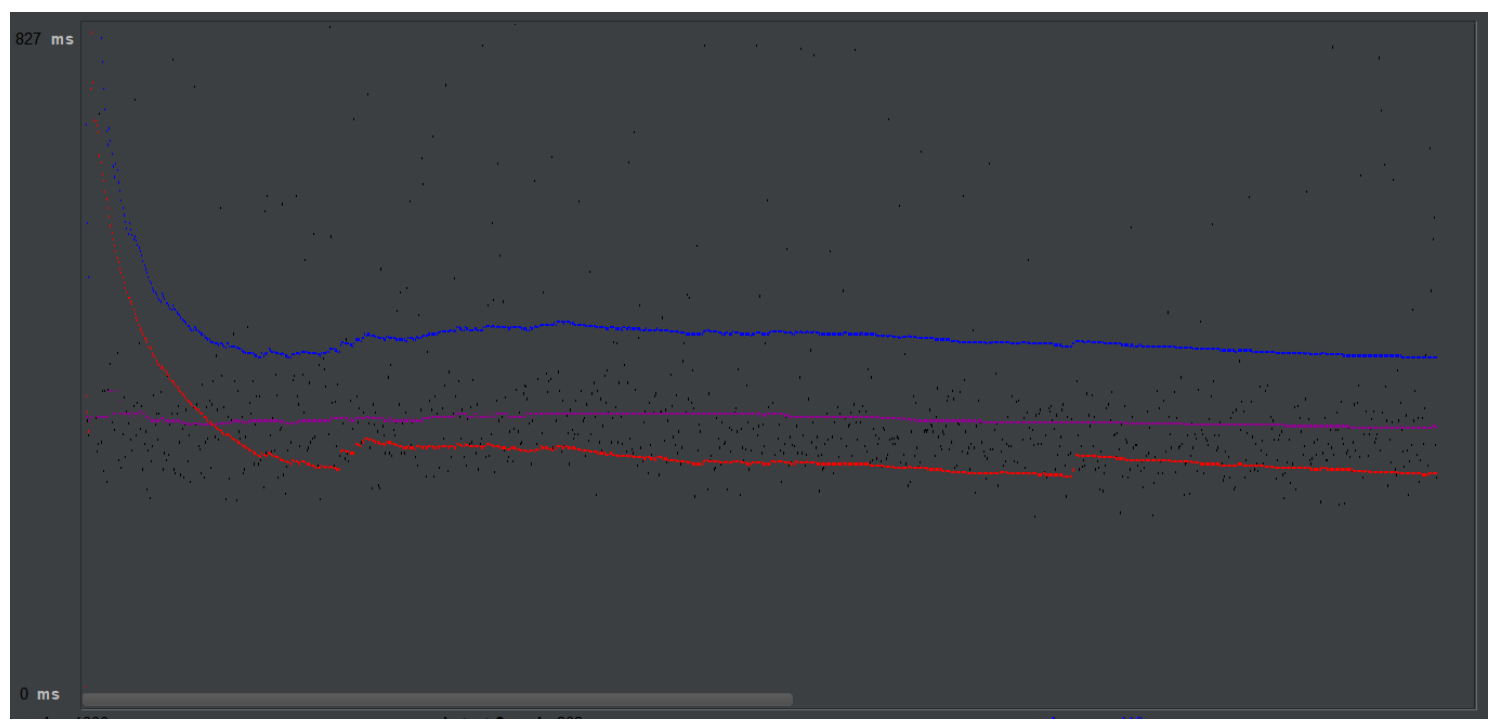

Abbildung 19: Graphisches Ergebnis Szenario 2 Google Cloud Run API.

Schwarz: Einzelne Antwortzeiten, Violett: Median, Blau: Mittelwert, Rot: Standardabweichung.

Quelle: Eigene Darstellung.

Am Graphen in Abbildung 19 ist zu erkennen, dass Standardabweichung und Mittlerwert der Antwortzeiten zu Beginn des Anfragenzeitraums höhere Werte haben und dann sinken. Nach einer gewissen Zeit pendeln sich die Linien ein. Dies liegt vermutlich an der Skalierung der Container-Instanzen von Google Cloud Run. Zudem liegen fast alle Antwortzeiten unter 1000 Millisekunden, der Mittelwert für alle 1000 Antwortzeiten beträgt 413 Millisekunden. Die CloudRessourcen sind ausreichend für die hier eingehenden Anfragen. 
6. Erweiterung der Java GEO Label API um die prototypische Generierung SSNO-basierter Label und Bereitstellung als Cloud-basierte serverless Anwendung

Ergebnis AWS Lambda (AWS Scenario 2 V1):

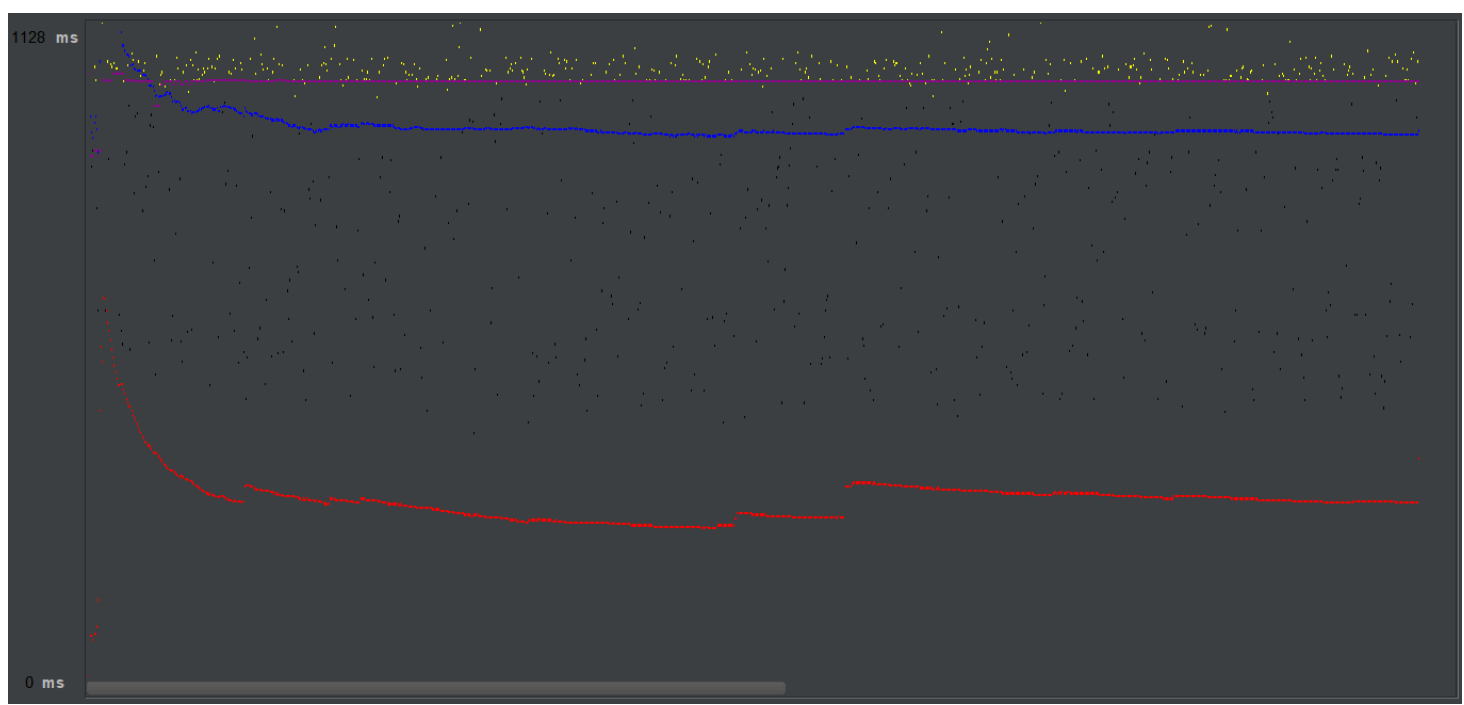

Abbildung 20: Graphisches Ergebnis Szenario 2 AWS Lambda API.

Schwarz/Gelb: Einzelne Antwortzeiten, Violett: Median, Blau: Mittelwert, Rot: Standardabweichung. Quelle: Eigene Darstellung.

Der Graph in Abbildung 20 zeigt, dass der Mittlerwert aller Antwortzeiten über den gesamten Anfragezeitraum hinweg nahe an 1000 Millisekunden liegt. Viele Antwortzeiten (hier nicht abgebildet) liegen sogar weit über 1000 Millisekunden. Zudem erkennt man, dass der Mittelwert und die Standardabweichung, die beide möglichst niedrig sein sollten, zu Beginn beide sehr hoch sind und dann sinken und sich einpendeln, was vermutlich an der automatischen Skalierung der Funktions-Instanzen von AWS Lambda liegt. Obwohl der Mittelwert für alle 1000 Anfragen mit 943 Millisekunden noch unter 1000 Millisekunden liegt, wird der Arbeitsspeicher der LambdaFunktion trotzdem auf 1 Gibibyte erhöht und das Szenario wiederholt. Das Ergebnis (AWS_Scenario_2_V2) ist in Abbildung 21 zu sehen:

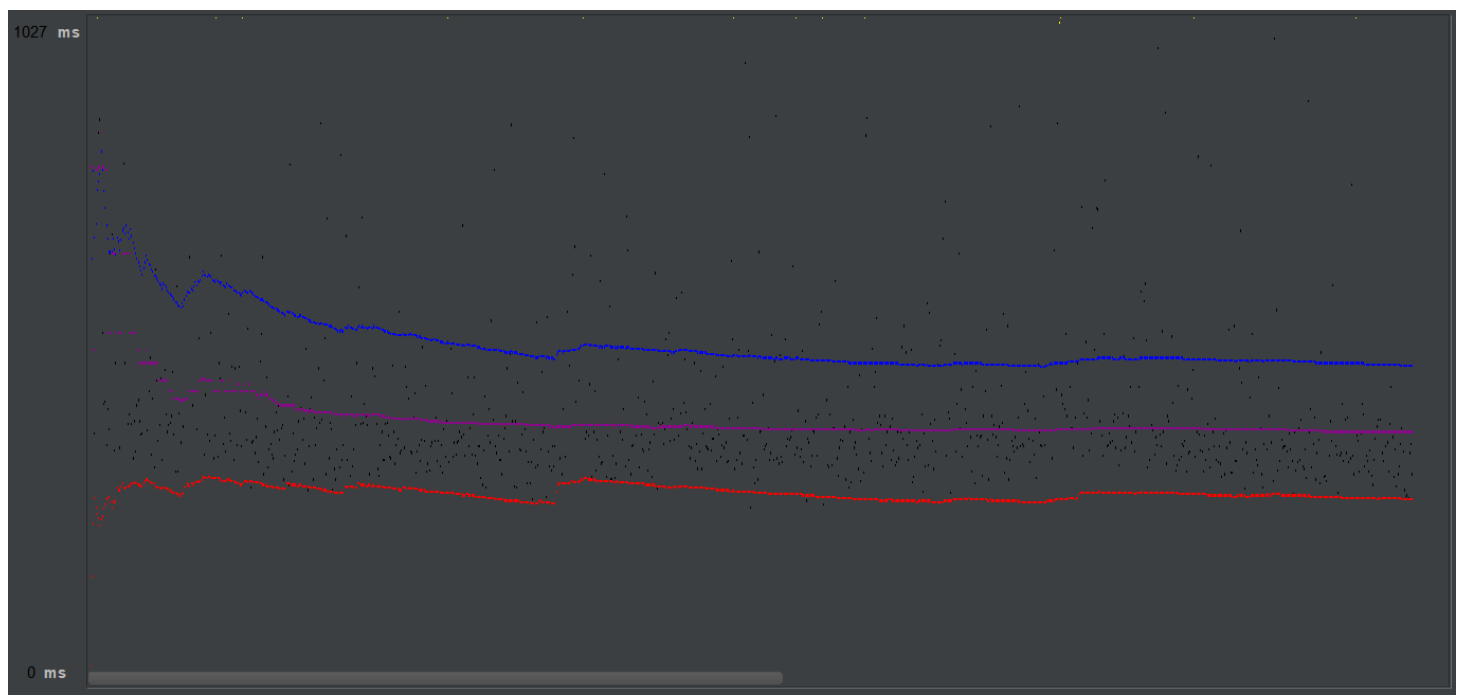

Abbildung 21: Graphisches Ergebnis Szenario 2 AWS Lambda API - Überarbeitete Cloud-Ressourcen. Schwarz/Gelb: Einzelne Antwortzeiten, Violett: Median, Blau: Mittelwert, Rot: Standardabweichung. Quelle: Eigene Darstellung.

Wie Abbildung 21 veranschaulicht, sind auch dieses Mal Median, Mittelwert und Standardabweichung am Anfang des Anfragezeitraums höher und sinken mit der Zeit, was 
6. Erweiterung der Java GEO Label API um die prototypische Generierung SSNO-basierter Label und Bereitstellung als Cloud-basierte serverless Anwendung

wieder auf die automatische Skalierung zurückzuführen ist. Allerdings ist dieses Mal nur ein kleiner Teil der Antwortzeiten länger als 1000 Millisekunden. Die anderen Antwortzeiten liegen in einem sehr guten Bereich, was man daran erkennt, dass alle drei Linien konstant niedrig liegen. Der Mittelwert aller Antwortzeiten beträgt sogar nur 476 Millisekunden. Für die nächsten Szenarien bleibt die neue Einstellung der Cloud-Ressourcen erhalten.

\section{Ergebnis Local Host (LH Scenario 2 V1):}

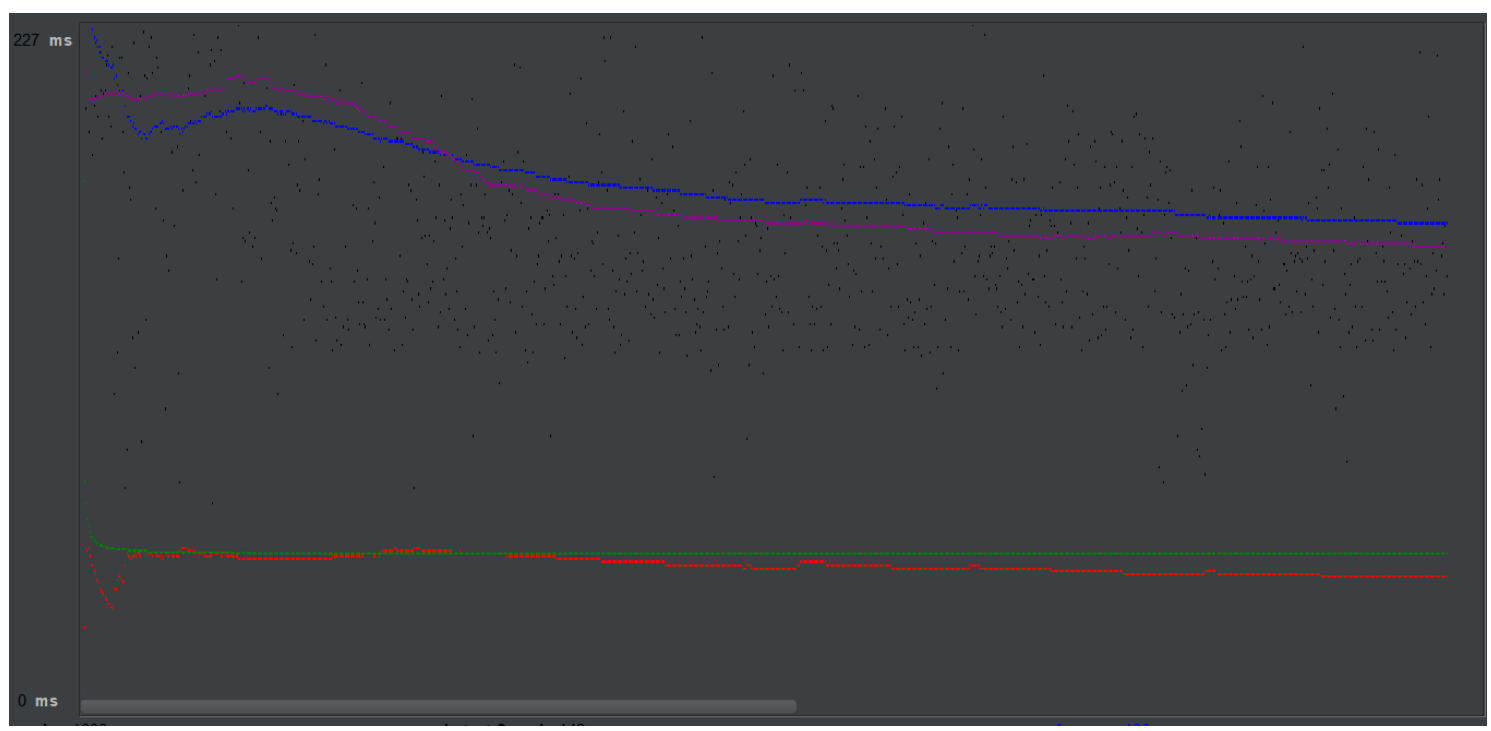

Abbildung 22: Graphisches Ergebnis Szenario 2 Local Host API.

Schwarz: Einzelne Antwortzeiten, Violett: Median, Blau: Mittelwert, Rot: Standardabweichung. Quelle: Eigene Darstellung.

Alle Antwortzeiten liegen unter 1000 Millisekunden. In Abbildung 22 ist zu sehen, dass Median und Mittelwert anfangs noch etwas höher sind, nach und nach aber sinken. Am Ende beträgt der Mittelwert für alle Anfragen 160 Millisekunden. Auch die Standardabweichung ist über den gesamten Zeitraum sehr gering. Diese niedrigen Werte zeigen, dass der Code der Implementierung für die GEO Label API schnell ausgeführt werden kann.

\section{Szenario 3 - Die Stadt Münster will für 100 Datensätze gleichzeitig Label generieren.}

Um das Szenario zu simulieren, werden in JMeter 100 Nutzer definiert, die alle gleichzeitig eine Anfrage senden, damit 100 Anfragen parallel abgeschickt werden.

Hierbei wird nicht mehr auf die einzelnen Antwortzeiten geachtet, sondern auf die Gesamtdauer zur Beantwortung aller Anfragen. Für jede API wird der Tests fünf Mal wiederholt.

\section{Ergebnis Google Cloud Run (GCR Scenario 3 V1):}

Einige Anfragen werden mit Status Code 500 Internal Server Error beantwortet. Auch in den Logs vom Google Cloud Run Dienst wird eine Fehlernachricht angezeigt: Memory limit of 256M exceeded with $256 \mathrm{M}$ used. Consider increasing the memory limit. Deshalb wird eine neue Revision erstellt und der Arbeitsspeicher auf 1 Gibibyte und die CPU auf 2 pro Containerinstanz erhöht. Dies kann natürlich auch zu einer Erhöhung der Kosten führen. Das Szenario wird erneut mit den neuen Einstellungen für die Cloud-Ressourcen ausgeführt (GCR_Scenario_3_V2): 
6. Erweiterung der Java GEO Label API um die prototypische Generierung SSNO-basierter Label und Bereitstellung als Cloud-basierte serverless Anwendung

Durchgang 1: 45 Sekunden

Durchgang 2: 39 Sekunden

Durchgang 3: 18 Sekunden

Durchgang 4: 15 Sekunden

Durchgang 5: 3 Sekunden

Bei allen Durchgängen wurden alle 100 Anfragen jeweils gültig beantwortet. Auch an diesem Szenario erkennt man, dass mit steigender Anzahl der eingehenden Anfragen die Antwortzeiten immer kürzer werden. Zunächst werden 100 Anfragen in 45 Sekunden beantwortet, beim letzten Durchgang sogar in 3 Sekunden.

Ergebnis AWS Lambda (AWS Scenario 3 V2):

Durchgang 1: 8 Sekunden

Durchgang 2: 1 Sekunde

Durchgang 3: 1 Sekunde

Durchgang 4: 1 Sekunde

Durchgang 5: 1 Sekunde

An diesem Ergebnis fällt auf, dass die Antwortzeiten deutlich geringer sind als bei Google Cloud Run. Beiden Arbeitsspeichern sind 1 Gibibyte zugeordnet. Zudem wird die Antwortzeit aller Anfragen in JMeter nur in Sekunden angegeben, die Zeiten für AWS Lambda könnten also auch unter 1 Sekunde gelegen haben.

\section{Ergebnis Local Host (LH Scenario 3 V1):}

Durchgang 1: 3 Sekunden

Durchgang 2: 3 Sekunden

Durchgang 3: 4 Sekunden

Durchgang 4: 3 Sekunden

Durchgang 5: 3 Sekunden

Da es für den lokalen Container keine automatische Skalierung gibt, sind die Antwortzeiten jedes Durchgangs ungefähr gleich.

Szenario 4 - Ein großer Online-Datenanbieter generiert einmal im Monat GEO Labels für seine neuen Daten. Dabei handelt es sich um ca. 1000 neue Datensätze monatlich.

\section{Ergebnis Google Cloud Run (GCR Scenario $4 \quad 1 \quad$ V2):}

Für einige Anfragen werden verschiedene Fehlernachrichten (vollständig im Anhang 10) angezeigt:

HTTP response code: javax.net.ssl.SSLHandshakeException, Non HTTP response message: Remote host terminated the handshake

HTTP response code: javax.net.ssl.SSLException, Non HTTP response message: Connection reset 
Alle Meldungen deuten erstmal laut verschiedenen Foreneinträgen ${ }^{113}$ und aufgrund der Tatsache, dass bei weniger gleichzeitigen Anfragen keine Probleme auftreten darauf hin, dass das System überlastet ist. In den Logs vom Cloud Run Dienst werden keine Fehler angezeigt, es scheint als würden die Anfragen gar nicht erst verarbeitet werden. Daher wird nun zunächst eine neue Revision für den Dienst erstellt mit verbesserten Einstellungen: CPU = 2 (Maximum), Arbeitsspeicher $=2$ Gibibyte (Maximum), Gleichzeitigkeit $=40$. Die Gleichzeitigkeit auf $1 \mathrm{zu}$ stellen wäre nicht sinnvoll, da insgesamt auch nur 1000 Container-Instanzen gleichzeigt generiert werden können. Auch diese Einstellungen könnten natürlich die Kosten deutlich erhöhen, wenn das kostenlose Kontingent aufgebraucht ist.

Trotz verbesserter Einstellungen ist das Ergebnis (GCR_Scenario_4_1_V3) nicht besser. Es werden dieselben Fehlernachrichten angezeigt, was darauf schließen lässt, dass der Dienst 1000 gleichzeitig gesendete Anfragen nicht verarbeiten kann. Deshalb wird die Anzahl der gleichzeitig gesendeten Anfragen nun jedes Mal um 100 verringert, wenn Fehler auftreten. Bei 600 gleichzeitigen Anfragen ist der Test erfolgreich (GCR_Scenario_4_2_V3). Alle 600 Anfragen werden in 51 Sekunden gültig beantwortet. Der Cloud Run Dienst könnte die 1000 Anfragen also gestückelt beantworten, indem immer höchsten 600 Anfragen gleichzeitig an den Dienst geschickt werden.

\section{Ergebnis AWS Lambda (AWS Scenario 41 V2):}

Bei AWS Lambda erscheinen dieselben Fehler wie bei Google Cloud Run. Deshalb wird hier der Arbeitsspeicher auf 3 Gibibyte erhöht (Maximum) und das Szenario wiederholt. Das Ergebnis (AWS_Scenario_4_1_V3) beinhaltet trotzdem noch dieselben Fehler. Dies lässt vermuten, dass die Fehler auch auf etwas anderes als auf die Skalierungen und Cloud-Ressourcen der Anbieter zurückzuführen sind.

Der Arbeitsspeicher wird wie bei Google Cloud Run zurück auf 2 Gibibyte gesetzt und die Anzahl der gleichzeitig gesendeten Anfragen nun auch jedes Mal um 100 verringert, wenn Fehler auftreten. Es können 300 gleichzeitige Anfragen in 9 Sekunden beantwortet werden (AWS_Scenario_4_2_V4), also weniger als bei Google Cloud Run. Aber auch hier könnte die Anfrage gestückelt werden, indem immer 300 Anfragen gleichzeitig geschickt werden.

\section{Ergebnis Local Host (LH Scenario 41 V1):}

Alle 1000 gleichzeitig gesendeten Anfragen werden in 30 Sekunden gültig beantwortet, es werden also 1000 Labels in 30 Sekunden generiert.

\footnotetext{
${ }^{113}$ Foreneinträge verfügbar unter:

https://user.jmeter.apache.narkive.com/qh19QJc2/remote-host-closed-connection-during-handshake, https://stackoverflow.com/a/31781529/12238641, https://stackoverflow.com/a/46309038/12238641.
} 


\section{Diskussion und Ausblick}

Im Rahmen dieser Bachelorarbeit wurde gezeigt, dass es möglich ist, die Java GEO Label API von 52 North zu erweitern und öffentlich bereitzustellen, um GEO Label basierend auf Sensorbeschreibungen zu generieren, die auf Technologien des semantischen Webs beruhen. Im Folgenden werden die einzelnen Ergebnisse, die im Laufe der Arbeit zur Beantwortung der Forschungsfragen gemacht wurden, diskutiert.

Für alle Facetten wurden Informationsquellen in SSNO oder anderen Ontologien gefunden. Neben SSNO bieten besonders PROV-O und DUV Klassen und Properties, die als Informationsquellen für die Facetten geeignet sind. Zwischen PROV-O und SSNO gab es bereits ein Ontology-Alignement, für DUV wurde in dieser Arbeit kein vollständiges Alignment, sondern nur Ansätze eines Alignments erstellt. Im Gegensatz zu den anderen Metadatenformaten, die bisher von der Implementierung der GEO Label API unterstützt wurden, können bei den SSNObasierten Sensorbeschreibungen auch die Feedback-Informationen in einer Sensorbeschreibung angegeben werden anstatt in einem externen Feedbackdokument. Einige Informationsquellen entsprechen allerdings nicht ganz den ursprünglichen Beschreibungen der Facetten. Für die Facette Producer Comments wird die Property rdfs:comment in einer Sensorbeschreibung gesucht. Laut Beschreibung der Facette soll es sich bei diesen Kommentaren um Angaben zur Qualität der Daten handeln. Allerdings kann mit rdfs: comment ein Kommentar zu allen Subjekten in einem RDF-Graphen gemacht werden. Es lässt sich also nicht eingrenzen, was genau dieser Kommentar beschreibt. Die Bedeutung der Facette Quality Information wird durch die gewählten Klassen und Properties erweitert. Dabei kann es Informationen zur Qualität des ganzen Systems geben, auf dem der Sensor basiert. Für die Zukunft sollten die Facettenbeschreibungen also angepasst werden oder die Facetten selbst überarbeitet werden.

Weiterhin gibt es neben den hier verwendeten Ontologien und Vokabularen sicherlich noch weitere Klassen und Properties aus anderen Ontologien und Vokabularen, mit denen Sensoren und ihre Beobachtungen beschrieben werden könnten. Eine Sensorbeschreibung könnte momentan also Informationen zu einer Facette enthalten, ohne dass diese ausgefüllt wird. Die Linked Data Ontologien sind daher nicht zu vergleichen mit den anderen Metadatenstandards, die eine feste Struktur und feste Datenfelder besitzen. Diese Tatsache führt zu einer weiteren Komplikation. Die Abfrage der Sensorbeschreibungen mit XPATH für die Generierung von Labels über die GEO Label API ist möglich, wenn die RDF-Graphen der Beschreibungen als RDF/XML serialisiert sind. Wie bereits beschrieben, gibt es mehrere Möglichkeiten zur Darstellung eines RDF-Graphen in RDF/XML. Diese unterschiedlichen Möglichkeiten müssen bereits bei der Erstellung eines XPATH-Ausdrucks berücksichtig werden, um Elemente an bestimmten Stellen im RDF/XML-Dokument zu finden. Dadurch werden die XPATH-Ausdrücke sehr lang. Darüber hinaus würden die Ausdrücke noch komplexer werden, wenn noch weitere Informationsquellen für die Facetten hinzugefügt werden sollten. Anstelle von XPATH könnte daher SPARQL als Abfragesprache verwendet werden, da diese Sprache auf Graphenmuster spezialisiert und deshalb nicht von der Struktur einer Serialisierung abhängig ist. Weiterhin ist SPARQL auch nicht von der Serialisierung selbst abhängig, das heißt, durch die Erweiterung der Implementierung der GEO Label API um SPARQL als Abfragesprache für Metadatendokumente aus dem semantischen Web könnten auch Sensorbeschreibungen in Turtle, Notation 3 und anderen Serialisierungen an die API geschickt werden. 
Darüber hinaus wurden für die Hover-Over-Texte der einzelnen Facetten Vorschläge gemacht, diese könnten in der Implementierung zusammen mit der Drilldown-Funktion auch noch umgesetzt werden.

Insgesamt macht das GEO Label selbst keine Angaben über die Qualität der Informationen in einem Metadatendokument, die durch die Facetten repräsentiert werden, sondern nur darüber, ob Informationen vorhanden sind oder nicht. Für SSNO-basierte Sensorbeschreibungen ist dies insofern problematisch, da diese auf Linked Data basieren. Zwar empfiehlt Tim Berners-Lee in seinen vier Regeln (Kapitel 2.1.1) HTTP-URIs für die Bezeichnung von Objekten zu verwenden, allerdings ist dies nicht fest vorgeschrieben. Das bedeutet, wenn ein URI in einer Sensorbeschreibung angegeben ist, der kein HTTP-URI ist (dieser also nicht nachgeschlagen werden kann) und zusätzlich auch keine Literale vorhanden sind, kann eine Facette trotzdem ausgefüllt werden, auch wenn keine wirklich qualitativen Informationen im Dokument enthalten sind. Für diesen Fall könnte der Visualisierungszustand Informationen sind auf einer höheren Ebene verfügbar in der Implementierung umgesetzt werden.

Für die Bereitstellung der GEO Label API wurden die Serverless Cloud-Computing Anbieter Google Cloud Run und AWS Lambda gewählt. Mit Google Cloud Run wurde der gesamte GEO Label Service online zur Verfügung gestellt, bei AWS Lambda hingegen wurde nur die Generierung von Labels als SVG unter Angabe von Metadaten- und/oder Feedback-URLS umgesetzt. In Zukunft könnte die Bereitstellung mit AWS Lambda noch vervollständigt werden.

Für beide Anbieter wurden dieselben Performanz-Tests durchgeführt. Begonnen wurde mit den jeweiligen Standardeinstellungen für die Cloud-Ressourcen, die je nach Nutzer-Szenario erhöht werden mussten. Zum Vergleich wurden die Szenarien auch für eine GEO Label API ausgeführt, die lokal in einem Tomcat-Docker-Container lief. Einzelne Anfragen konnten alle APIs meist in unter 1000 Millisekunden beantworten, auch wenn mehrere Anfragen in Abständen von mindestens 1 Sekunde eingingen. Dabei fiel auf, dass bei den APIs, die über die Serverless CloudComputing Anbieter bereitgestellt wurden, die Antwortzeiten kürzer wurden mit steigender Anzahl an Anfragen. Dies ist auf die automatische Skalierung beider Serverless Cloud-Computing Anbieter zurückzuführen.

Zudem konnten auch 100 gleichzeitige Anfragen von allen APIs bearbeitet werden. Auch hier wurde die gesamte Bearbeitungszeit mit wachsender Anzahl an Anfragen kürzer. Die Bearbeitungszeiten lagen für 100 Anfragen teilweise im einstelligen Sekundenbereich. Die Zeiten für AWS Lambda waren sogar etwas kürzer als die für Google Cloud Run. Bei 1000 gleichzeitigen Anfragen erreichten beide Serverless Cloud-Computing APIs ihre Grenzen. Auch mit maximalen Cloud-Ressourcen, die je nach Anbieter möglich sind, konnten 1000 gleichzeitige Anfragen nicht ohne Fehler verarbeitet werden. Die lokale API hingegen konnte 1000 simultan gesendete Anfragen verarbeiten. Die Implementierung selbst ist also dazu in der Lage, die Anzahl an gleichzeitigen Anfragen zu beantworten. Zudem kann eine Überlastung der Hardware weitestgehend ausgeschlossen werden, auf der die Tests ausgeführt werden.

Letztendlich wurden für Google Cloud Run folgende Cloud-Ressourcen festgesetzt: $\mathrm{CPU}=2$, Arbeitsspeicher $=2$ Gibibyte, Gleichzeitigkeit $=40$. Für die API, die mit AWS Lambda und Amazon API Gateway zur Verfügung gestellt wurde, wurde schließlich ein Arbeitsspeicher von 2 Gibibyte festgesetzt. Für die Gleichzeitigkeit wurde die Standardeinstellung 1000 beibehalten. Da dieser Wert für alle Lambda-Funktionen eines AWS Accounts gilt, lässt sich diese Einstellung nicht mit 
der von Google Cloud Run vergleichen, bei der die Gleichzeitigkeit pro Dienst gilt. Um eine größere Anzahl von Labels zu generieren, müssen Anfragen gestückelt an die APIs gesendet werden. Mit den letztlich eingestellten Cloud-Ressourcen können 600 Anfragen gleichzeitig an die Google Cloud Run API gesendet werden und 300 Anfragen an die AWS Lambda API. Bei AWS Lambda könnte der Arbeitsspeicher für die Lambda-Funktion noch auf maximal 3 Gibibyte erhöht werden.

Die Ergebnisse zeigen zunächst, dass sich die Funktionsweisen der Serverless Cloud Computing Anbieter zwar ähneln, sich aber in einigen Dingen auch grundlegend unterscheiden. Trotzdem ist kein Anbieter hinsichtlich Performanz bei der Generierung von GEO Labels grundlegend besser bzw. schlechter als der andere. Zusätzlich gibt es noch weitere Dinge, die die Performanz der bereitgestellten APIs beeinflussen und im Rahmen dieser Arbeit nicht berücksichtigt wurden. Der Java Code selbst braucht je nach Implementierung eine gewisse Zeit, um vollständig ausgeführt zu werden. Darüber hinaus wird bei Google Cloud Run und auch bei der lokalen Bereitstellung ein Tomcat-Docker-Container verwendet. Auch hier gibt es bestimmte Limits.

Trotzdem waren die Bereitstellungen der GEO Label API mit beiden Anbietern erfolgreich. Für diese Art von Anwendung ist die Verwendung eines Serverless Cloud-Computing Anbieters zur Bereitstellung sicherlich sinnvoll, da nicht ununterbrochen Anfragen an die GEO Label API eingehen würden und somit Kosten gespart werden würden. Die Ergebnisse der Nutzerszenarien zeigen, dass sich die automatischen Skalierungen gut an die Anzahl der ankommenden Anfragen anpassen. Lediglich bei größeren Anzahlen gleichzeitiger Anfragen sind die Grenzen erreicht, diese Szenarien sind für die GEO Label API aber auch eher unwahrscheinlich.

\section{Zusammenfassung}

Das Ziel der Arbeit, durch die Erweiterung der GEO Label API dem Nutzer von Sensordaten ein Label zur Verfügung zu stellen, das dem Nutzer eine Übersicht über die Verfügbarkeit von Informationen in SSNO-basierten Sensorbeschreibungen gibt, konnte erreicht werden.

Für die Facetten des GEO Labels wurden zunächst die Semantic Sensor Network Ontology sowie die verbundenen Ontologien evaluiert, um Informationsquellen für die Facetten auszumachen. Für alle Facetten wurden Quellen gefunden. Einige Facetten wurden in ihren Bedeutungen erweitert.

Die Java-Implementierung der GEO Label API von 52 North wurde anschließend um die Generierung SSNO-basierter Labels erweitert, indem eine neue Transformationsdatei für SSNObasierte Metadatendokumente hinzugefügt wurde. Die Implementierung unterstützt nur SSNObasierte RDF-Graphen als RDF/XML-Serialisierung, da so weiterhin XPATH zur Abfrage der Informationen für die Facetten des Labels verwendet werden konnte. Für die Zukunft soll ein Wechsel zu SPARQL als Abfrage für Metadatendokumente des semantischen Webs in Betracht gezogen werden. Die Hover-Over-Texte und Drilldown-Funktionen wurden zudem auch noch nicht in der Implementierung umgesetzt.

Neben Google Cloud Run wurde die weiterentwickelte GEO Label API auch mit AWS Lambda bzw. Amazon API Gateway bereitgestellt. Beide Bereitstellungen waren erfolgreich. AWS 
Lambda unterstützt aktuell nur die Generierung von Labels als Scalable Vector Graphic auf Basis von angegebenen URLs zu den Metadaten- und Feedbackdokumenten. Beide Anwendungen ähneln sich in ihrer Performanz. Die Ergebnisse der Nutzerszenarien entsprachen weitestgehend den Erwartungen, nur größere Mengen gleichzeitiger Anfragen konnten nicht mehr fehlerlos verarbeitet werden. Die Cloud-Ressourcen (Arbeitsspeicher, CPU, Gleichzeitigkeit) wurden anhand der Nutzerszenarien angepasst. Die Möglichkeit der Einstellung der Cloud-Ressourcen bietet dem Entwickler trotzdem nicht die vollständige Kontrolle über die Performanz eines Cloud-Dienstes.

Insgesamt wurden in dieser Arbeit die jüngeren Technologien des semantischen Webs und des Cloud-Computings erfolgreich dazu verwendet, dem Nutzer von Sensordaten die Suche nach den Daten zu vereinfachen. Dabei wurde neben dem eigentlichen Ziel, die Java GEO Label API von 52 North zu erweitern, auch die Möglichkeit der Integration dieser jüngeren Technologien in bereits bestehende Anwendungen in den Fokus gesetzt. 


\section{Literaturverzeichnis}

Amazon Web Services (2020a): Funktionen von AWS Lambda, https://aws.amazon.com/de/lambda/features/, Zugriff am 29.01.2020.

Amazon Web Services (2020b): Managing Concurrency for a Lambda Function, https://docs.aws.amazon.com/lambda/latest/dg/configuration-concurrency.html, Zugriff am 06.02.2020.

Amazon Web Services (2020c): AWS Lambda - Preise, https://aws.amazon.com/de/lambda/pricing/, Zugriff am 05.02.2020.

Amazon Web Services (2020d): API Gateway, https://aws.amazon.com/de/api-gateway/, Zugriff am 05.02.2020.

Antoniou, G. \& Harmelen, F. (2003): Web ontology Language: OWL. In: Staab S. \& Studer R. (Hrsg.): Handbook on Ontologies, S. 67-92, http://doi.org/10.1007/978-3-540-92673-3_4, Zugriff am 04.01.2020.

Berners-Lee, T. (2006): Linked Data, http://www.w3.org/Designlssues/LinkedData.html, Zugriff am 30.12.2019.

Data on the Web Working Group (2016): Data on the Web Best Practices: Dataset Usage Vocabulary, https://www.w3.org/TR/2016/NOTE-vocab-duv-20161215/, Zugriff am 23.01.2020.

Dataset Exchange Working Group (2019): Data Catalog Vocabulary (DCAT) - Version 2, https://www.w3.org/TR/2019/PR-vocab-dcat-2-20191119/, Zugriff am 23.01.2020.

Dengel, A. (Hrsg.) (2012): Semantische Technologien. Grundlagen - Konzepte - Anwendungen, 1. Auflage, Heidelberg: Spektrum Akademischer Verlag.

GeoViQua Consortium (2013): D.7.4 GCl pilot case perspective recommendations for enabling quality aware visualisation search, http://www.geoviqua.org/Docs/SubmittedDeliverables/D7_4_GeoViQua.pdf, Zugriff am 21.11.2019.

GIS-Technologie, Interoperabilität, IT, Standards (o.J.): SensorWeb, http://www.gis-news.de/technologie/sensorweb/, Zugriff am 04.01.2020.

Google (o.J.a): Cloud Run, https://cloud.google.com/run/?hl=de, Zugriff am 29.01.2020.

Google (o.J.b): Google Cloud Platform, https://cloud.google.com/docs/overview/?hl=de, Zugriff am 29.01.2020.

Google (o.J.c): Kostenlose Stufe Google Cloud, https://cloud.google.com/free/docs/gcp-free-tier?hl=de, Zugriff am 29.01.2020. 
Google (o.J.d): Ressourcenmodell, https://cloud.google.com/run/docs/resource-model?hl=de, Zugriff am 30.01.2020.

Google (o.J.e): Gleichzeitigkeit, https://cloud.google.com/run/docs/about-concurrency?hl=de, Zugriff am 30.01.2020.

Google (o.J.f): Speicherlimits konfigurieren, https://cloud.google.com/run/docs/configuring/memory-limits?hl=de, Zugriff am 30.01.2020.

Google (o.J.g): Schnellstart: Erstellen und Bereitstellen, https://cloud.google.com/run/docs/quickstarts/build-and-deploy?hl=de, Zugriff am 01.02.2020.

Gradmann, S. / Hennicke, S. / Olensky, M. (2012): Linked Data. In: Digitale Dienste für die Wissenschaft, Nr. 35, S. 18 - 22, http://doi.org/10.18452/6627, Zugriff am 31.12.2019.

Group on Earth Observations (o.J.a): GEO Community, https://www.earthobservations.org/geo_community.php, Zugriff am 16.11.2019.

Group on Earth Observations (o.J.b): GEOSS, https://www.earthobservations.org/geoss.php, Zugriff am 20.11.2019.

Group on Earth Observations (o.J.c): About, http://www.geolabel.info/About.htm, Zugriff am 21.11.2019.

Haller, A. / Janowicz, K. / Cox, S. / Lefrancois, M. / Taylor, K. / Phuoc, D. / Liebermann, J. / GarciaCastro, R. / Atkinson, R. / Stadler, C. (2019): The Modular SSN Ontology: A Joint W3C and OGC Standard Specifying the Semantics of Sensors, Observations, Sampling, and Actuation. In: Semantic Web, Nr. 10, S. 9-33, http://armin-haller.com/publications/SSN-SWJ.pdf, Zugriff am 10.01.2020.

Hitzler, P. / Krötzsch, M. / Rudolph, S. / Sure, Y. (2008): Semantic Web, 1. Auflage, Berlin: Springer.

IBM (2020): Serverunabhängige REST-APIs erstellen, https://cloud.ibm.com/docs/openwhisk?topic=cloud-functions-apigateway, Zugriff am 29.01.2020.

Microsoft (2020): Was ist Cloud Computing?, https://azure.microsoft.com/de-de/overview/what-is-cloud-computing/, Zugriff am 20.01.2020.

Microsoft Azure (2017): HTTP-Trigger und -Bindungen in Azure Functions, https://docs.microsoft.com/de-de/azure/azure-functions/functions-bindings-http-webhook?ta bs=csharp, Zugriff am 29.01.2020.

Nielsen, J. (1993): Usability Engineering, Kapitel 5, https://www.nngroup.com/articles/response-times-3-important-limits/, Zugriff am 30.01.2020. 
Nüst, D. \& Lush, V. (2015): A GEO label for the Sensor Web, https://doi.org/10.31223/osf.io/ka38z, Zugriff am 05.01.2020.

Open Geospatial Consortium (2020): Sensor Web Enablement DWG, https://www.opengeospatial.org/projects/groups/sensorwebdwg, Zugriff am 04.01.2020.

Open Geospatial Consortium (2020): Welcome to The Open Geospatial Consortium, https://www.opengeospatial.org/, Zugriff am 04.01.2020.

Oracle (2019): Was ist das Internet of Things?, https://www.oracle.com/de/internet-of-things/what-is-iot.html, Zugriff am 04.01.2020.

$\begin{array}{llll}\text { o.V. } & \text { (o.J.): World Wide }\end{array}$ https://www.gruenderszene.de/lexikon/begriffe/world-wide-web?interstitial, Zugriff am 29.11.2019.

Provenance Working Group (2013): PROV-O: The PROV Ontology, https://www.w3.org/TR/2013/REC-prov-o-20130430/, Zugriff am 22.01.2020.

Refsnes Data (o.J.): XPath Tutorial, https://www.w3schools.com/xml/xpath_intro.asp, Zugriff am 04.01.2020.

Shet, A. / Henson, C. / Sahoo, S. (2008): Semantic Sensor Web. In: Internet Computing, IEEE, Nr. 12, S. 78-83, http://doi.org/10.1109/MIC.2008.87, Zugriff am 16.01.2020.

Shotton, D. \& Peroni, S. (2013): BiRO, the Bibliographic Reference Ontology, https://sparontologies.github.io/biro/current/biro.html, Zugriff am 26.01.2020.

Spatial Data on the Web Working Group (2017): Semantic Sensor Network Ontology, http://www.w3.org/TR/2017/REC-vocab-ssn-20171019/, Zugriff am 10.01.2020.

World Wide Web Consortium (2019): About W3C, https://www.w3.org/Consortium/, Zugriff am 29.11.2019.

World Wide Web Consortium (2015): Linked Data, http://www.w3.org/standards/semanticweb/data, Zugriff am 30.12.2019.

World Wide Web Consortium (2019): Standards, http://www.w3.org/standards/, Zugriff am 31.12.2019.

World Wide Web Consortium (2012): OWL 2 Web Ontology Language. Document Overview (Second Edition), http://www.w3.org/TR/2012/REC-owl2-overview-20121211/, Zugriff am 04.01.2020.

World Wide Web Consortium (2015): XML Essentials, http://www.w3.org/standards/xml/core, Zugriff am 01.01.2020. 


\section{Anhang}

\section{Anhang 1: Beispielseite des 52 North GEO Label Service}

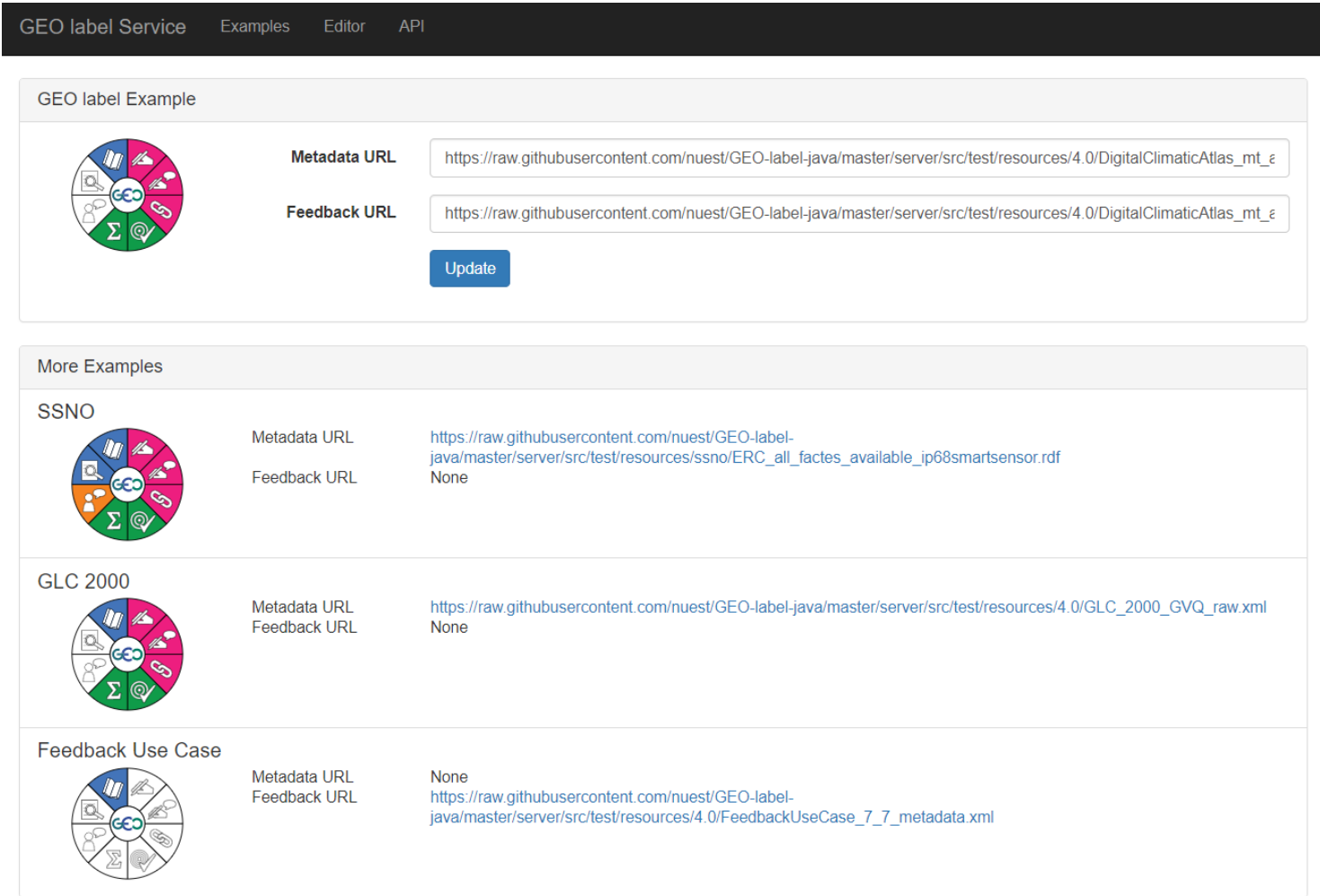


Anhang 2: Bearbeitungsseite des 52 North GEO Label Service GEO label Service Examples Editor API

\begin{tabular}{l} 
GEO label \\
Enter metadata and/or feedback document and \\
click "Update" to view the corresponding GEO \\
label representation. \\
\hline
\end{tabular}




\section{Anhang 3: RDF/XML-Serialisierungen erstellt durch den Easy RDF Converter - Datei ERC_all_factes_available_ip68smartsensor.rdf}

$<? \mathrm{xml}$ version="1.0" encoding="utf-8" ?>

- This file is based on a sensor description published at https://whow w3.org/TR/vocab-5sn/\#ip68-smart-sensor.

$<$ rdf:RDF xmlns:rdf="http://www.w3.org/1999/02/22-rdf-syntax-ns\#"

xmlns: org="http://www.w3.org/ns/org\#"

xmlns : owl="http://www.w3.org/2002/07/owl\#"

xmlns:rdfs="http://www.w3.org/2000/01/rdf-schema\#"

xmlns:gr="http://purl.org/goodrelations/v1\#"

xmlns:ssn-systems="http://Www.w3.org/ns/ssn/systems/"

xmlns: schema="http://schema.org/"

xmlns : ssn="http://www.w3.org/ns/ssn/"

xmlns: sosa="http://www.w3.org/ns/sosa/

xmlns: prov="http://www.w3.org/ns/prov\#"

xmlns : foaf="http://xmlns. com/foaf/0.1/"

xmlns:geo="http://www.w3.org/2003/01/geo/wgs84_pos\#"

xmlns: duv="http://www.w3.org/ns/duv\#"

xmlns:oa="http://WWw. W3.org/ns/oa\#"

xmins:skos=" hte://pur.ws.org/2004/02/sko

xins:

<org:Organization rdf:about="https://data.grandlyon.com/Organization/1">

<owl: sameAs rdf:resource="http://dbpedia.org/page/Metropolis_of_Lyon"/>

$</$ org:Organization>

$\langle$ rdf:Description rdf:about="https://data.grandlyon.com/Air"

$\langle$ rdf:type rdf:resource="http://www.w3.org/ns/sosa/Feature0fInterest"/>

$\langle$ rdfs:label xml:lang="en" $\rangle$ The air. $\langle/$ rdfs $:$ label $\rangle$

$</$ rdf:Description

<owl:Class rdf:about="https://data.grandlyon.com/IP68_Outdoor_Temperature_Sensor"

ServiceModel"/>

$\langle\mathrm{gr}$ : name $\mathrm{xml}$ : lang="en" $>$ IP68 Outdoor Temperature Sensor $\langle/ \mathrm{gr}$ : name $\rangle$

$\langle$ rdfs:label xml:lang="en">IP68 Outdoor Temperature Sensor $\langle/$ rdfs:label $\rangle$

$\langle$ rdfs: subClassof $\rangle$

$<$ rdf:Description>
<owl: onProperty

<owl: onProperty $\mathrm{rdf}:$ : resource="http://ww.w3.org/ns/ssn/systems/hasOperatingRange"/>

¿wl: hasValue>

$\langle$ rdf:Description rdf:about="https://data.grandlyon.com/IP68_Outdoor_Temperature_Sensor\#operatingRange" rdf: type $\mathrm{rdf}:$ resource="http://www.w3.org/ns/ssn/systems/OperatingRange"/>

作 $\langle$ rdf:Description>

$</$ rdf:Desch

$\langle$ rdfs: subClassOf>

$\langle$ rdfs: subClassOf

$\langle$ rdf:Description

<owl: onProperty rdf: resource="http://www.w3.org/ns/ssn/systems/hasSystemCapability"/

<owl: hasValue

$\langle$ rdf:Description rdf:about="https://data.grandlyon.com/IP68_Outdoor_Temperature_Sensor\#systemCapability"

<rdf: type rdf: resource="http://ww.w3.org/ns/ssn/Property"/>

$\langle$ rdf:type rdf:resource="http://ww.w3.org/ns/ssn/systems/SystemCapability"/>

$\langle$ rdfs:comment xhl:lang="en" $>$ The sensor capability in normal operating conditions.</rdfs:comment

〈ssn-systems: hasSystemProperty>

$\langle$ rdf:Description rdf:about="https://data.grandlyon.com/IP68_Outdoor_Temperature_Sensor\#RFSensitivity">

$\langle$ rdf:type rdf: resource="http://www.w3.org/ns/ssn/Property"/>

$<$ rdf: type $r d f:$ resource="http://www. w3. org/ns/ssn/Property"
$\langle$ rdf:type rdf: resource="http://www. w3.org/ns/ssn/systems/S

<rdf:type rdf: resource="http://schema.org/ ropertylalue"/>

$</$ rdf:Description $>$

$</ s s n-$ systems: hasSystemProperty

ssn-systems: hasSystemProperty>

〈rdf:Description rdf:about="https://data.grandlyon.com/IP68_Outdoor_Temperature_Sensor\#TemperatureAccuracy" >

〈rdf:type rdf: resource="http://www.w3.org/ns/ssn/Property"/>

$\langle$ rdf: type rdf: resource= $=$ http://www.wi.org/ns/ssn/systems/Acc

$\langle$ rdf:type rdf:resource="http://schema.org/PropertyValue"/>

ssn:forProperty $\mathrm{rdf}$ : resource="https://data. grandlyon. com/Air? 1 lat=45.75\&amp;long=4.85\#temperature" $/\rangle$

<schema:minValue rdf:datatype="http://www.w3.org/2001/XMLSchema\#decimal">- $0.2</$ schema:minValue

〈schema:maxValue rdf:datatype="http://wwW.W3.org/2001/XMLSchema\#decimal">0.2</schema:maxValue

<schema: unitCode rdf: resource="http://qudt.org/vocab/unit\#DegreeCelsius"/

$</ s$ sn-systems : has SystemProperty

<s:

://data.grandlyon.com/IP68_Outdoor_Temperature_Sensor\#TemperatureResolution">

iresource="http://wwl.w3.org/ns/ssn/systems/Resolution"//

df: type rdf: resource="http://schema.org/PropertyValue"/>

<ssn:forProperty $\mathrm{rdf}:$ resource="https://data. grandlyon.com/Air?lat=45.75\&amp;long=4.85\#temperature"/>

〈schema:value rdf:datatype="http://www.w3.org/2001/XMLSchema\#decimal">0.0625</schema:value〉

<schema: unitcode

$</$ ssn-systems: hasSystemProperty

〈ssn-systems: hasSystemProperty rdf: resource="https://data.grandlyon.com/IP68_Outdoor_Temperature_Sensor\#BatteryAccuracy"/> 〈ssn-systems: hasSystemproperty>

〈ssn:Property rdf:about="https://data.grandlyon.com/IP68_Outdoor_Temperature_Sensor\#BatteryResolution">

$\langle$ rdf: type $r d f:$ resource="http://wWl.w3.org/ns/ssn/systems/Resolution"/>

〈rdf: type rdf: resource="http://schema.org/PropertyValue"/>

(2)

schema:value rdf:datatype="http://wWl.w3.org/2001/XMLSchema\#double" $>3.937 \mathrm{e}-3</$ schema:value

<schema:unitCode rdf: resource="http://qudt.org/vocab/unit\#Percent"/

$\langle$ ssn: Property

$\langle$ ssn-systems: hasSystemProperty

ssn-systems:inCondition $\mathrm{rdf}:$ resource="https://data.grandlyon.com/IP68_Outdoor_Temperature_Sensor\#normalOperatingCondition"/> $/$ rdf:Description

$<$ owl: has Value

$</$ rdf:Description $>$

$\langle/$ owl:Class $>$ 
〈ssn-systems:Condition rdf:about="https://data.grandlyon.com/IP68_Outdoor_Temperature_Sensor\#normalOperatingCondition"> 〈rdf:type rdf:resource="http://schema.org/PropertyValue"/>

renture range of -20 to 70 Celsius $</ \mathrm{rdfs}:$ comment

〈schema:minValue rdf: datatype="http://www.w3.org/2001/XMLSchema\#decimal">-20.0</schema:minValue >

〈schema:maxValue rdf:datatype="http://Www.w3.org/2001/XMLSchema\#decimal">70.0</schema:maxValue〉

<schema:unitCode rdf:resource="http://qudt.org/vocab/unit\#DegreeCelsius"/>

$</$ ssn-systems:Condition

〈rdf:Description rdf: about="https://data.grandlyon.com/Air?lat=45.75\&amp;long=4.85"> $\langle$ rdf:type rdf:resource="http://www.w3.org/ns/sosa/Sample"/>

$\langle$ rdfs:label xml:lang="en" >The air at lat 45.75 and long $4.85,\langle/ \mathrm{rdfs}: 1$ label $\rangle$

<sosa:isSampleOf rdf: resource="https://data.grandlyon.com/Air"/>

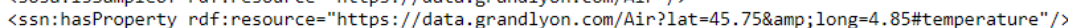

$</$ rdf:Description $>$

〈ssn:Property $\mathrm{rdf}$ : about="https://data.grandlyon.com/Air?lat=45.75\&amp;long=4.85\#temperature">

<rdf:type rdf:resource="http://www.w3.org/ns/sosa/ObservableProperty"/>

<ssn:isPropertyof $\mathrm{rdf}:$ resource="https://data.grandlyon. com/Air?lat=45.75\&amp;long=4.85"/>

$</ s s n:$ Property

$\langle\mathrm{gr}$ :ProductOrService $\mathrm{rdf}$ : about="https://data.grandlyon.com/Sensor/SL-T-P1"

$\langle$ rdf:type rdf:resource="http://www.w3.org/ns/sosa/Sensor"/>

$\langle$ rdf:type rdf:resource="http://www.w3.org/ns/prov\#Entity"/>

〈rdf:type rdf:resource="https://w3id.org/seas/LoRaCommunicationDevice"/>

$\langle$ rdf:type rdf:resource="https://data.grandlyon.com/IP68_Outdoor_Temperature_Sensor"/> $\langle$ prov:wasAttributedTo

<prov:Agent rdf:about="https://data.grandlyon.com/Organization/exampleOrganization">

$\langle$ rdf:type rdf:resource="http://www.w3.org/ns/prov\#0rganization"/>

$\langle$ foaf:name>Example Organization</foaf:name>

$</$ prov: Agent $>$

$</$ prov:wasAttributedTo

$<\mathrm{gr}$ : hasBrand

$\langle$ gr: Brand>

$\langle\mathrm{gr}$ : name $\mathrm{xml}:$ lang="en" $>$ Sensing Labs $</ \mathrm{gr}$ : name $>$

$\langle/ \mathrm{gr}:$ Brand $\rangle$

$\langle/ \mathrm{gr}$ : hasBrand $>$

<geo:alt rdf:datatype="http://www.w3.org/2001/XMLSchema\#decimal" $>100.0</$ geo:alt

$\langle$ geo:lat rdf: datatype="http://wWw.w3.org/2001/XMLSchema\#decimal" $>45.75</$ geo: lat $>$

〈geo:lon rdf:datatype="http://www.w3.org/2001/XMLSchema\#decimal">4.85</geo: lon〉

〈ssn:implements rdf:resource="https://data.grandlyon.com/IP68_Outdoor_Temperature_Sensor\#temperatureSensingProcedure"/>

〈ssn:implements rdf: resource="https://data.grandlyon.com/IP68_Outdoor_Temperature_Sensor\#tbatterySensingProcedure"/>

〈ssn:observes rdf: resource="https://data.grandlyon.com/Sensor/SL-T-P1\#battery"/>

〈ssn: observes $\mathrm{rdf}:$ resource="https://data.grandlyon.com/Air?lat=45.75\&amp;long=4.85\#temperature"/>

$\langle/$ gr: ProductOrService

〈ssn:Deployment rdf:about="https://data.grandlyon.com/Deployment/SL-T-P1/2017-06-06">

〈ssn:deployedSystem $\mathrm{rdf}$ : resource="https://data.grandlyon.com/Sensor/SL-T-P1"/>

〈prov: startedAtTime rdf:datatype="http://www.w3.org/2001/XMLSchema\#date">2017-06-06</prov: startedAtTime>

<prov:wasAssociatedWith rdf:resource="https://data.grandlyon.com/Organization/1"/

$\langle/$ ssn:Deployment

〈sosa:Observation rdf:about="https://data.grandlyon.com/Observation/5872357\#temperature">

$\langle$ rdf:type rdf: resource="http://wWw.w3.org/ns/dcat\#Dataset"/>

$\langle$ rdf:type rdf:resource="http://wnu,w3.org/ns/prov\#Activity"/>

<duv: hasFeedback

〈duv: RatingFeedback rdf:about="https://data.grandlyon.com/rating/1">

<oa: hasBody>

〈skos:Concept rdf:about="https://data.grandlyon.com/good">

〈skos:inscheme $\mathrm{rdf}$ : resource="https://data.grandlyon.com/rating"/>

<skos:prefLabel xml:lang="en">good</skos:prefLabel>

$</$ skos: Concept

$</$ oa: hasBody

$<$ oa: hasTarget rdf: resource="https://data.grandlyon.com/Observation/5872357\#temperature"/>

<oa:motivatedBy $\mathrm{rdf}$ : resource="http://www.w3.org/ns/oa\#assessing"/>

$</$ duv: RatingFeedback>

$\langle/$ duv: hasFeedback $>$

<duv: hasFeedback rdf: resource="https://data.grandlyon. com/comment/1"/>

<duv: hasFeedback

〈duv:UserFeedback rdf:about="https://data grandlyon.com/content/2">

$\langle$ rdf:type rdf:resource="http://www.w3.org/ns/prov\#Entity"/>

<oa:hasBody rdf:datatype="http://Www w3.org/2001/XMLSchema\#string" >This is a comment of an expert.</oa:hasBody>

$<\mathrm{oa}$ : hasTarget $\mathrm{rdf}:$ resource="https ://data. grandlyon.com/Observation/7536"/>

<prov:qualifiedAssociation>

<prov: Association>

$\langle$ prov: agent>

$\langle$ prov:Agent rdf: about="https://data.grandlyon.com/Person/exampleMan"〉

$\langle$ rdf: type rdf: resource="http://www.w3.org/ns/prov\#Person"/>

$\langle$ foaf:givenName>Man</foaf: givenName>

$\langle/$ prov:Agent $>$

$\langle/$ prov:agent $\rangle$

〈prov:Role rdf:about="https://data.grandlyon.com/expert"

$\langle/$ prov:Role $\rangle$

$\langle/$ prov:hadRole $\rangle$

$</$ prov:Association $>$

$</$ prov: qualifiedAssociation

$</$ duv:UserFeedback

$</$ duv: hasFeedback $>$

〈sosa: hasSimpleResult rdf:datatype="http://w3id.org/lindt/custom_datatypes\#ucum">64.5244681928429 Cel</sosa:hasSimpleResult >

〈sosa: madeBySensor $\mathrm{rdf}$ : resource="https://data.grandlyon.com/Sensor/SL-T-P1"/>

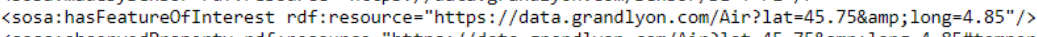

<sosa: observedProperty $\mathrm{rdf}:$ resource="https://data.grandlyon. com/Air?lat=45.75\&amp;long=4.85\#temperature"/>

<sosa:resultTime rdf:datatype="http://WwW:W3.org/2001/XMLSchema\#dateTime">2017-06-20T21:49:18+00:00</sosa: resultTime>
</sosa:Observation>

〈sosa:Observation rdf:about="https://data.grandlyon.com/Observation/5872357\#battery"〉

$\langle$ rdf:type rdf:resource="http://www.w3.org/ns/dcat\#Dataset"/>

<duv: hasFeedback rdf: resource="https://data.grandlyon.com/comment/1"/>

$\langle$ biro:isReferencedBy>

rdf:Description rdf:about="https://data.grandlyon.com/paper">

$\langle$ rdf:type rdf: resource="http://purl.org/spar/fabio/ResearchPaper"/>

$</$ rdf:Description>

$</$ biro:isReferencedB $>$

$\langle$ sosa:hasSimpleResult $\mathrm{rdf}$ : datatype="http://w3id.org/lindt/custom_datatypes\#ucum" $>73.2 \%</$ sosa:hasSimpleResult

<sosa:madeBySensor rdf: resource="https://data grandyon .com/Sensor/SL-T-P1"/> 
〈sosa: hasFeatureOfInterest rdf:resource="https://data.grandlyon.com/Sensor/SL-T-P1"/>

<sosa: observedProperty rdf: resource="https://data.grandlyon.com/Sensor/SL-T-P1\#battery"/>

<sosa:resultTime rdf:datatype="http://Www.w3.org/2001/XMLSchema\#dateTime">2017-06-20T21:49:18+00:00</sosa:resultTime>

$</$ sosa:Observation

〈skos:Concept rdf:about="https://data.grandlyon.com/bad">

$\langle$ skos: inscheme rdf: resource="https://data.grandlyon.com/rating"/〉

〈skos: prefLabel xml:lang="en">bad</skos:prefLabel>

$\langle/$ skos:Concept $\rangle$

〈skos:ConceptScheme rdf:about="https://data.grandlyon.com/rating"〉

<skos:prefLabel>A set of values to rate a observation.</skos:prefLabel

$</$ skos:ConceptScheme>

<duv:UserFeedback rdf:about="https://data.grandlyon.com/comment/1">

Koa:hasBody rdf:datatype="http://wwW.w3.org/2001/XNLSchema\#string">This is a comment of an user.</oa:hasBody>

<oa:hasTarget rdf:resource="https:///data.grandlyon.com/Observation/5872357/temperature"/>

<oa: hasTarget rdf:resource="https://data.grandlyon.com/Observation/5872357\#battery"/>

$\langle$ de:creator>

prov:Agent rdf:about="https://data.grandlyon.com/Person/exampleWoman">

$\langle$ rdf:type rdf:resource="http://www.w3.org/ns/prov\#Person"/>

$<$ foaf: givenName>Woman</foaf: givenName>

$\langle/$ dc: creator $>$
$\langle/$ duv:UserFeedback

$\langle/ r d f: R D F\rangle$

Anmerkung: Die Datei ist verfügbar unter https://github.com/anikagraupner/GEO-labeljava/blob/master/testdata/All_Facets_Available/RDFXML/ERC_EasyRDFConverter/ERC_all_fac tes_available_ip68smartsensor.rdf. Sie enthält Informationen zu allen Facetten. 


\section{Anhang 4: RDF/XML-Serialisierung erstellt durch den MyBluemix Validator and Converter Datei MBC_all_factes_available_ip68smartsensor.rdf}

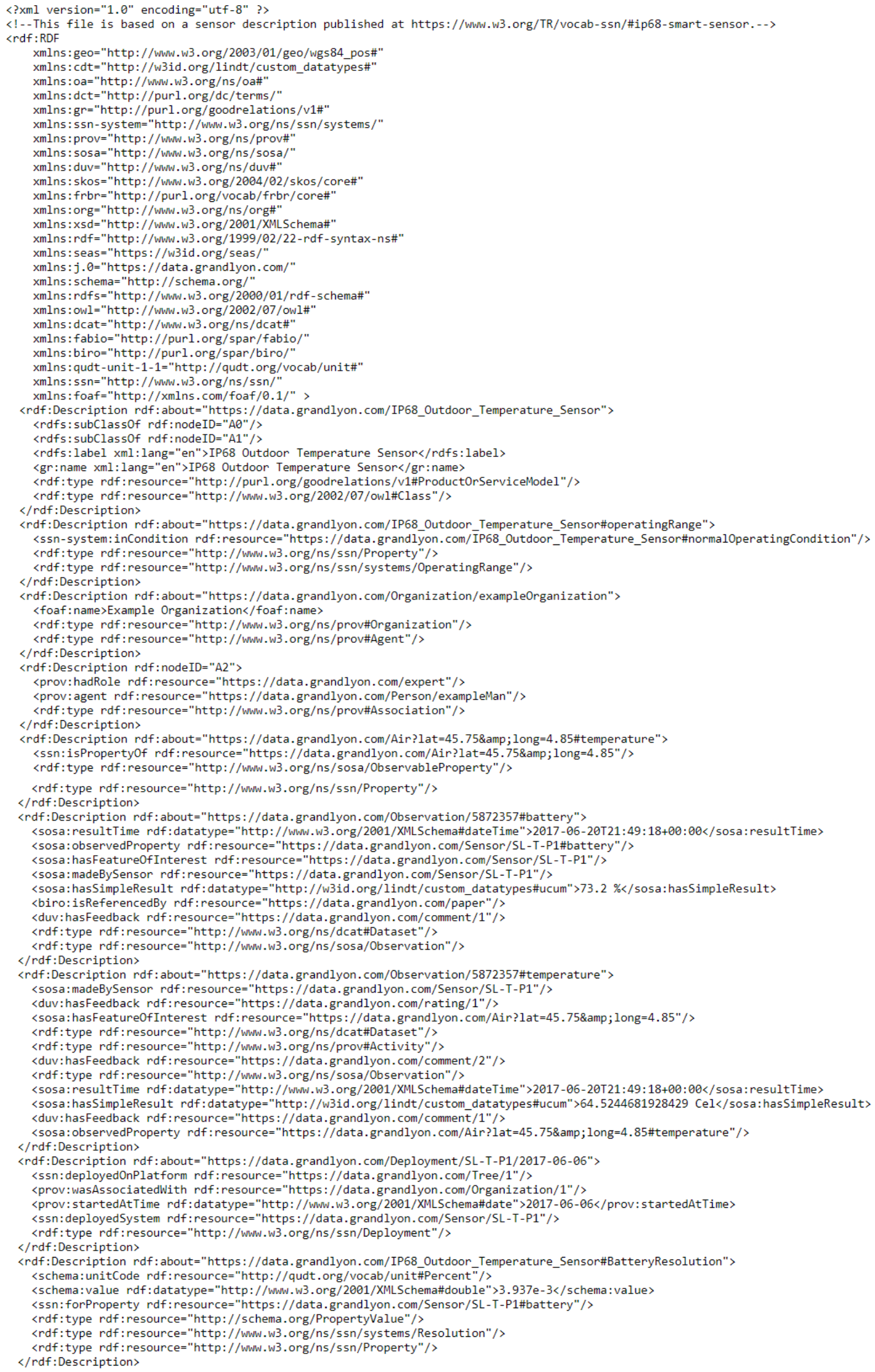




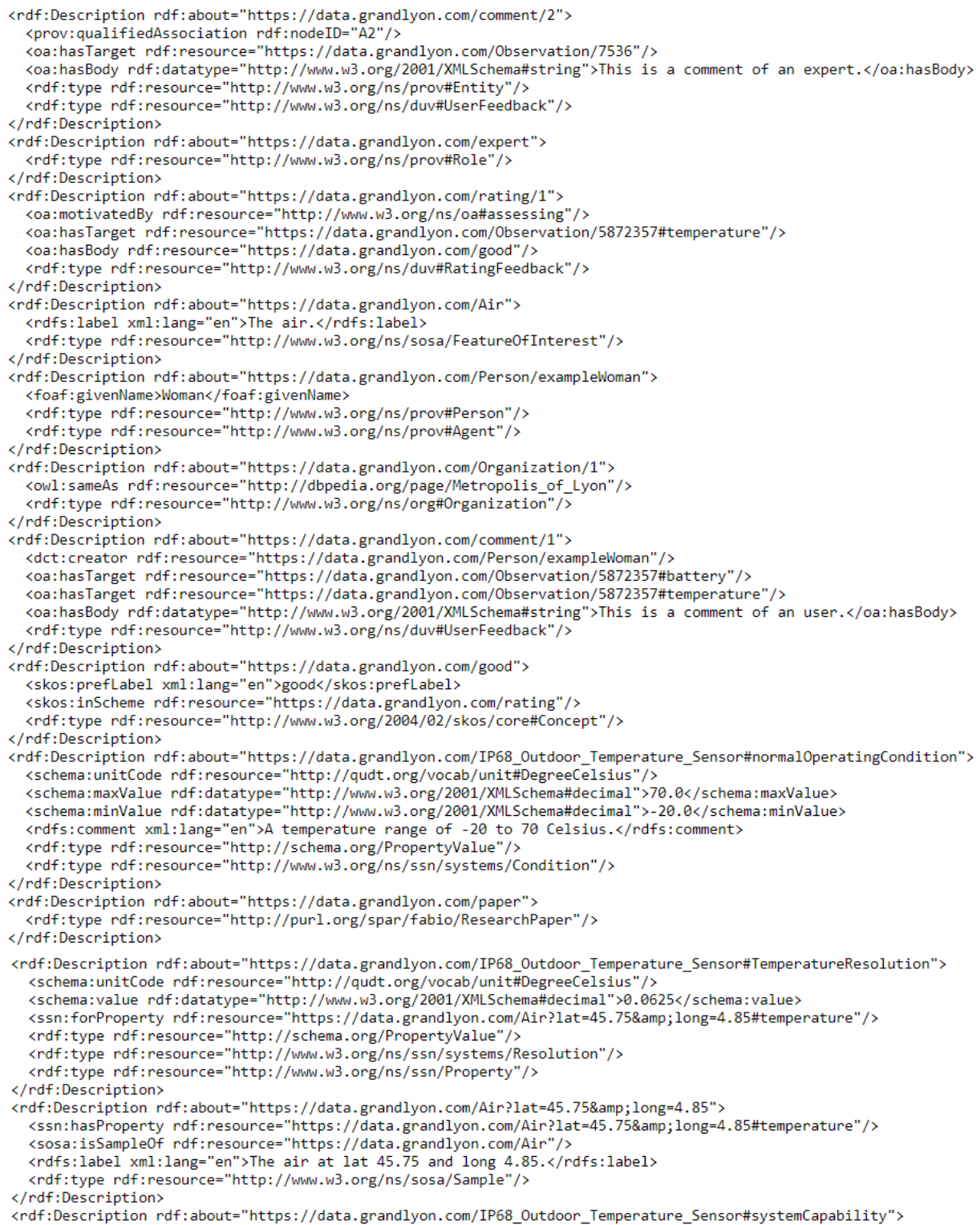

〈rdf:Description rdf:about="https://data.grandlyon.com/IP68_Outdoor_Temperature_Sensor\#systemCapability"〉

〈ssn-system:inCondition rdf:resource="https://data.grandlyon.com/IP68_Outdoor_Temperature_Sensor\#normalOperatingCondition"/> <ssn-system:hasSystemProperty rdf:resource="https://data.grandlyon.com/IP68_Outdoor_Temperature_Sensor\#BatteryResolution"/> 〈ssn-system: hasSystemProperty rdf: resource="https://data.grandyon.com/IP68_Outdoor_Temperature_Sensor\#BatteryAccuracy"/> 〈ssn-system:hasSystemProperty rdf:resource="https://data.grandlyon.com/IP68_Outdoor_Temperature_Sensor\#TemperatureResolution"/> 〈ssn-system: hasSystemProperty $\mathrm{rdf}:$ resource="https://data.grandyon.com/IP68_Outdoor_Temperature_Sensor\#TemperatureAccuracy"// $\langle$ rdfs:comment xml:lang="en">The sensor capability in n $\langle$ rdf:type rdf:resource="http://www.w3.org/ns/ssn/systems/SystemCapability"/>

<rdf:type rdf:resource="http://Www.w3.org/ns/ssn/Property"/>

$\langle$ rdf:Description rdf:about="https://data.grandlyon.com/IP68_Outdoor_Temperature_Sensor\#RFSensitivity" >

〈schema:unitCode rdf:resource="http://qudt.org/vocab/unit\#DecibelReferredToOneMilliwatt"/>

<schema:value rdf:datatype="http://wwlw,w3.org/2001/XMLSchema\#integer">-137</schema:value>

<rdf:type rdf: :esource="http://schema.org/PropertyValue"/>

$\langle$ rdf:type rdf:resource="http://www.w3.org/ns/ssn/systems/Sensitivity"/>

$\langle$ rdf:type rdf:resource="http://wwlw, w3.org/ns/ssn/Property"/>

$\langle$ rdf:Description rdf:about="https://data.grandlyon.com/Person/exampleMan"〉

$<$ foaf: givenName $>$ Man</foaf: givenName $>$

〈rdf: type rdf: resource="http://www.w3.org/ns/prov\#Person"/>

$\langle$ rdf: type rdf: resource="http://WwW.w3.org/ns/prov\#Agent"/>

$</$ rdf:Description $>$

$\langle$ rdf:Description rdf: about="https://data.grandlyon.com/bad"

<skos:prefLabel xml:lang="en">bad</skos:prefLabel>

<skos: inscheme rdf: resource="https://data.grandlyon.com/rating"/>

<rdf:type rdf:resource="http://www.w3.org/2004/02/skos/core\#Concept"/>

$</ r d f$ :Description $>$

$\langle$ rdf:Description rdf:about="https://data.grandlyon.com/rating">

<skos:prefLabel>A set of values to rate a observation.</skos:prefLabel

$\langle$ rdf:type rdf:resource="http://www.w3.org/2004/02/skos/core\#ConceptScheme"/

$\langle/ r d f:$ Description $\rangle$

$\langle$ rdf:Description rdf:about="https://data.grandlyon.com/IP68_Outdoor_Temperature_Sensor\#TemperatureAccuracy"

<schema:unitCode rdf:resource="http://qudt.org/vocab/unit\#DegreeCelsius"/

〈schema:maxValue $\mathrm{rdf}:$ datatype="http://Ww.w.w3.org/2001/XMLSchema\#decimal" $\rangle .2\langle/$ schema:maxValue $\rangle$

〈schema:minValue rdf:datatype="http://www.w3.org/2001/XMLSchema\#decimal">- $0.2</$ schema:minValue

〈ssn:forProperty $\mathrm{rdf}$ : resource="https://data.grandlyon.com/Air?lat=45.75\&amp;long=4.85\#temperature" />

〈rdf:type rdf:resource="http://schema.org/PropertyValue"/> 
<rdf:type rdf: resource="http://www.w3.org/ns/ssn/systems/Accuracy"/>

$\langle$ rdf:type rdf:resource="http://Www.w3.org/ns/ssn/Property"/>

$</$ rdf:Description>

$\langle$ rdf:Description rdf: nodeID="A0">

〈owl: hasValue rdf:resource="https://data.grandlyon.com/IP68_Outdoor_Temperature_Sensor\#systemCapability"/>

<owl:onProperty rdf:resource="http://Www.w3.org/ns/ssn/systems/hasSystemCapability"/>

$\langle/$ rdf:Description $>$

$\langle$ rdf:Description rdf: nodeID="A1"

<owl: hasValue rdf:resource="https://data.grandlyon.com/IP68_Outdoor_Temperature_Sensor\#operatingRange"/>

<owl: onProperty rdf: resource="http://wWw.w3.org/ns/ssn/systems/hasOperatingRange"/>

$</ r d f:$ Description $\rangle$

$\langle$ rdf:Description $r d f:$ nodeID="A3" $>$

$\langle\mathrm{gr}$ : name $\mathrm{xml}$ : lang="en">Sensing Labs $</ \mathrm{gr}$ : name $>$

$\langle$ rdf:type rdf:resource="http://purl.org/goodrelations/v1\#Brand"/>

$</$ rdf:Description $>$

rdf:Description rdf: about="https://data.grandlyon.com/Sensor/SL-T-P1"

〈ssn:implements rdf:resource="https://data.grandlyon.com/IP68_Outdoor_Temperature_Sensor\#temperatureSensingProcedure"/>

$\langle$ rdf: type rdf:resource="https://data.grandlyon.com/IP68_Outdoor_Temperature_Sensor"/

<geo:lat rdf: datatype="http://Www.w3.org/2001/XMLSchema\#decimal">45.75</geo:lat>

〈geo:alt rdf:datatype="http://wWw.w3.org/2001/XMLSchema\#decimal">100.0</geo:alt >

〈ssn: observes rdf: resource="https://data. grandlyon.com/Air?lat=45.75\&amp;long=4.85\#temperature" /

<geo:lon rdf: datatype="http://WWW.W3.org/2001/XMLSchema\#decimal">4.85</geo:lon

$\langle$ rdf: type rdf:resource="http://www.w3.org/ns/prov\#Entity"/>

〈rdf:type rdf: resource="http://purl.org/goodrelations/v1\#ProductOrService"/>

$\langle$ rdf: type rdf: resource="http://www.w3.org/ns/sosa/Sensor"/>

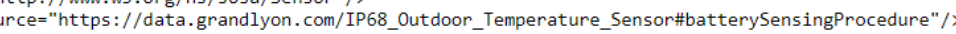

$\langle$ rdf:type rdf:resource="https://W3id.org/seas/LoRaCommunicationDevice"/>

Zation/exampleOrganization"/>

$<\mathrm{gr}$ : hasBrand raf: nodeID="A3"/>

$</$ rdf:Description $>$

Anmerkung: Die Datei ist verfügbar unter https://github.com/anikagraupner/GEO-labeljava/blob/master/testdata/All_Facets_Available/RDFXML/MBC_MyBluemixConverter/MBC_all _factes_available_ip68smartsensor.rdf. Sie enthält Informationen zu allen Facetten. 


\section{Anhang 5: Datei server.properties}

transformer. useLocalOnly=true

transformer. resources=http://geoviqua. github.io/geolabel/mappings/transformer. json=/transformations/transformer. json, $/$

http://geoviqua.github.io/geolabel/mappings/transformerSML101.json=/transformations/transformerSML101.json, 1

http://geoviqua.github.io/geolabel/mappings/transformerS0S20.json=/transformations/transformerS0S20.json, 1

http://geoviqua.github.io/geolabel/mappings/transformerSML20.json=/transformations/transformerSML20.json, /

http://geoviqua.github.io/geolabel/mappings/transformerSSNO.json=/transformations/transformerSSNO.json

cache. labels. $\max =100$

cache. hours . $\max =48$

drilldown.external.endpoint=http://localhost:8080/api/v1/drilldown

Anmerkung: Die Datei ist verfügbar unter https://github.com/anikagraupner/GEO-labeljava/blob/master/server/src/main/resources/server.properties. 


\section{Anhang 6: Datei transformerSSNO.json}

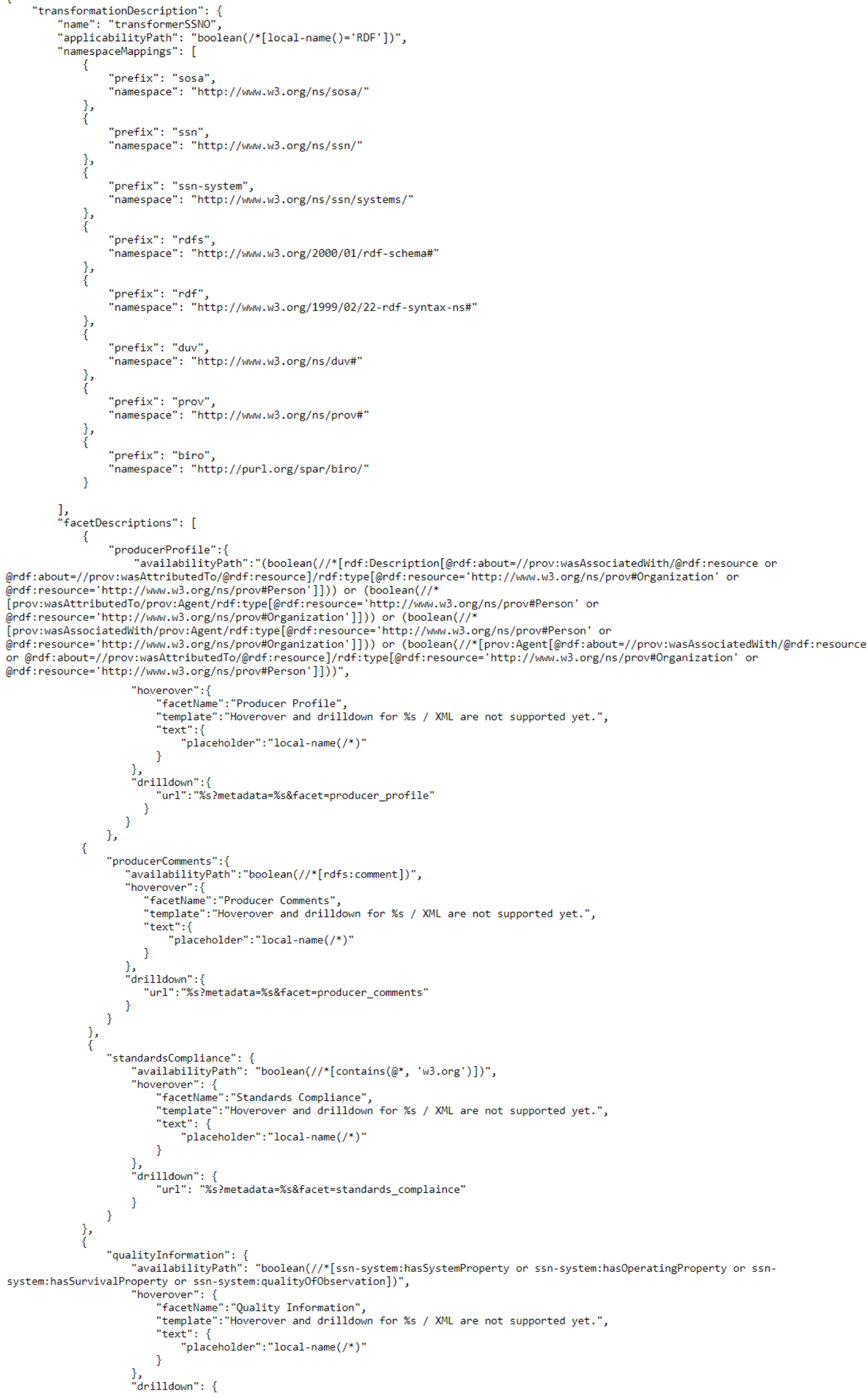




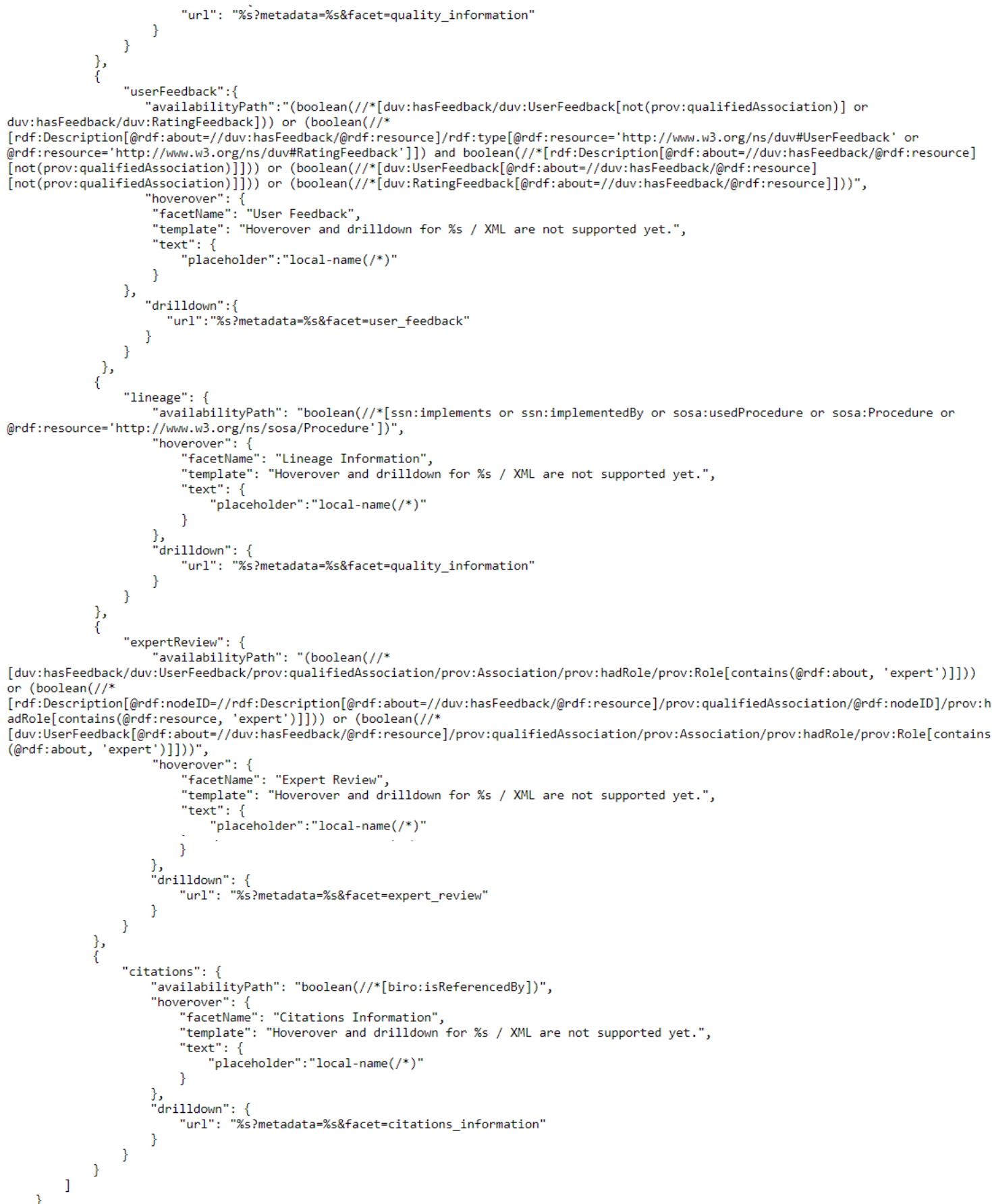

Anmerkung: Die Datei ist verfügbar unter https://github.com/anikagraupner/GEO-labeljava/blob/master/server/src/main/resources/transformations/transformerSSNO.json. 


\section{Anhang 7: Beispiele für \\ MetadataTransformerTest_SSNO.java

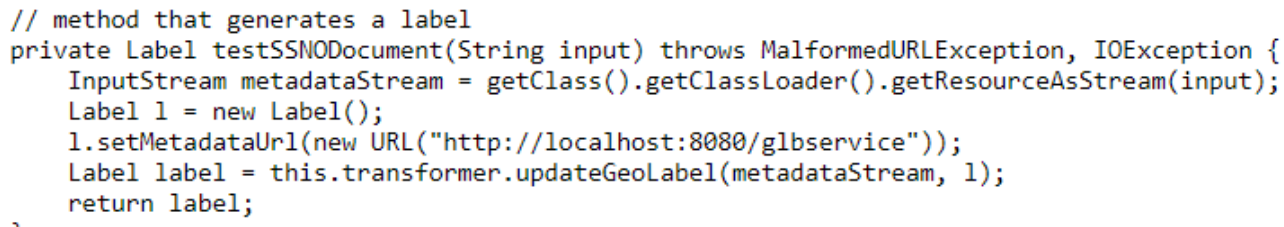

QTest //tests if the xpath works also with onknown prefixes, so that the namespace mapping must be execute public void unknownPrefixes() throws IOException \{

//EasyRDFConverter RDF/XML encoding

Label label = testSSNODocument("ssno/ERC_all_factes_available_ip68smartsensor_unknown_prefixes.rdf");

assertThat("standards compliance is found",

label.getStandardsComplianceFacet().getAvailability(),

equalTo(Availability.AVAILABLE));

assertThat("producer profile is found",

label.getProducerProfileFacet().getAvailability(),

equalTo(Availability.AVAILABLE));

assertThat("producer comment is found",

label.getProducerCommentsFacet().getAvailability(),

equalTo(Availability.AVAILABLE));

assertThat ("Quality information is found", label.getQualityInformationfacet().getAvailability(), equalTo(Availability.AVAILABLE));

assertThat ("User feedback is found", label.getUserFeedbackFacet().getAvailability(), equalTo(Availability.AVAILABLE));

assertThat("Lineage information is found", label.getLineageFacet().getAvailability(), equalTo(Availability.AVAILABLE));

assertThat("Expert feedback is found", label.getExpertFeedbackFacet().getAvailability(), equalTo(Availability.AVAILABLE));

assertThat("Citations information is found", label.getCitationsFacet().getAvailability(), equalTo(Availability.AVAILABLE)); 
@Test // tests first if the xpath find information about all facets and creates then an label and saves it local, the path is shown in the terminal public void create abelAllFacets() throws IOException

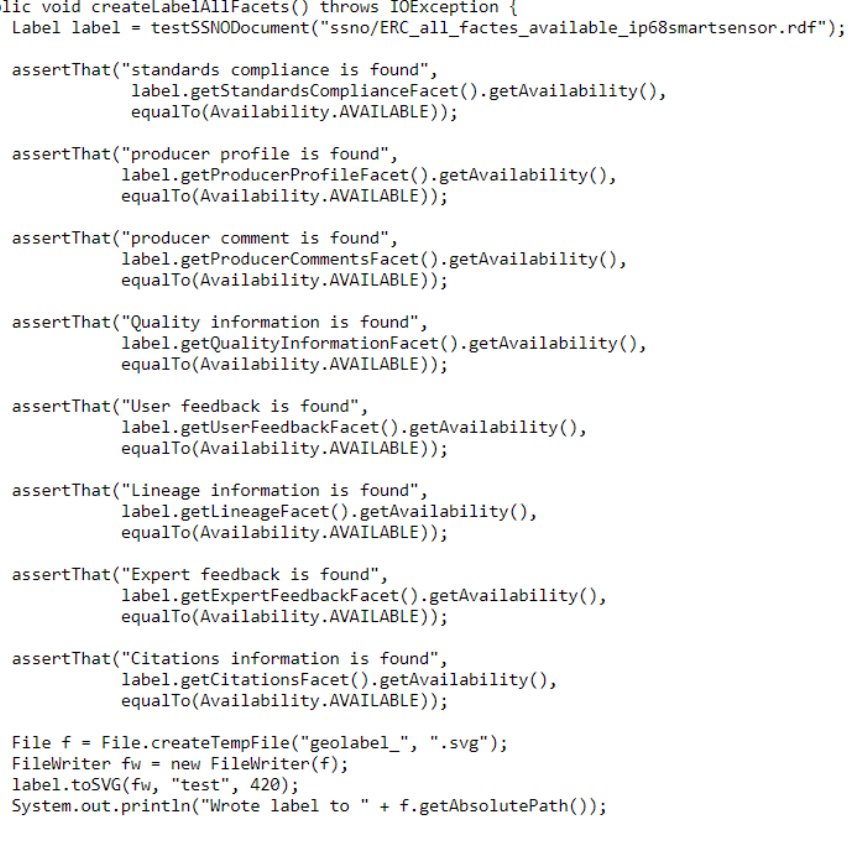

Anmerkung: Die Datei ist verfügbar unter https://github.com/anikagraupner/GEO-labeljava/blob/master/server/src/test/java/org/n52/geolabel/server/mapping/MetadataTransform erTest_SSNO.java. In dieser sind mehr Tests enthalten als die hier aufgeführten. 


\section{$\begin{array}{lllll}\text { Anhang } & \text { 8: } & \text { Testergebnisse } & \text { Unittests } & \text { Testklasse }\end{array}$ \\ MetadataTransformerTest_SSNO}

三 Java Test Report $\times$

All 12 Passed 12

org.n52.geolabel.server.mapping.MetadataTransformerTest_SSNO

> onlyStandardCompliance

Passed 908ms

$>$ unknownPrefixes

Passed 53ms

$>$ userFeedback

Passed $62 \mathrm{~ms}$

$>$ allFacets

Passed $\quad 89 \mathrm{~ms}$

> producerProfile

Passed $55 \mathrm{~ms}$

$>$ expertReviews

Passed 53ms

> producerComments

Passed 59ms

> allFacetsWithoutExpert

Passed $\quad 99 \mathrm{~ms}$

$>$ lineageAndQuality

Passed

$67 \mathrm{~ms}$

> createLabelOnlyStandardCompliance

Passed $688 \mathrm{~ms}$

citationsInformation

Passed $\quad 37 \mathrm{~ms}$

createLabelAllFacets 


\section{Anhang 9: Datei APIHandler.java}

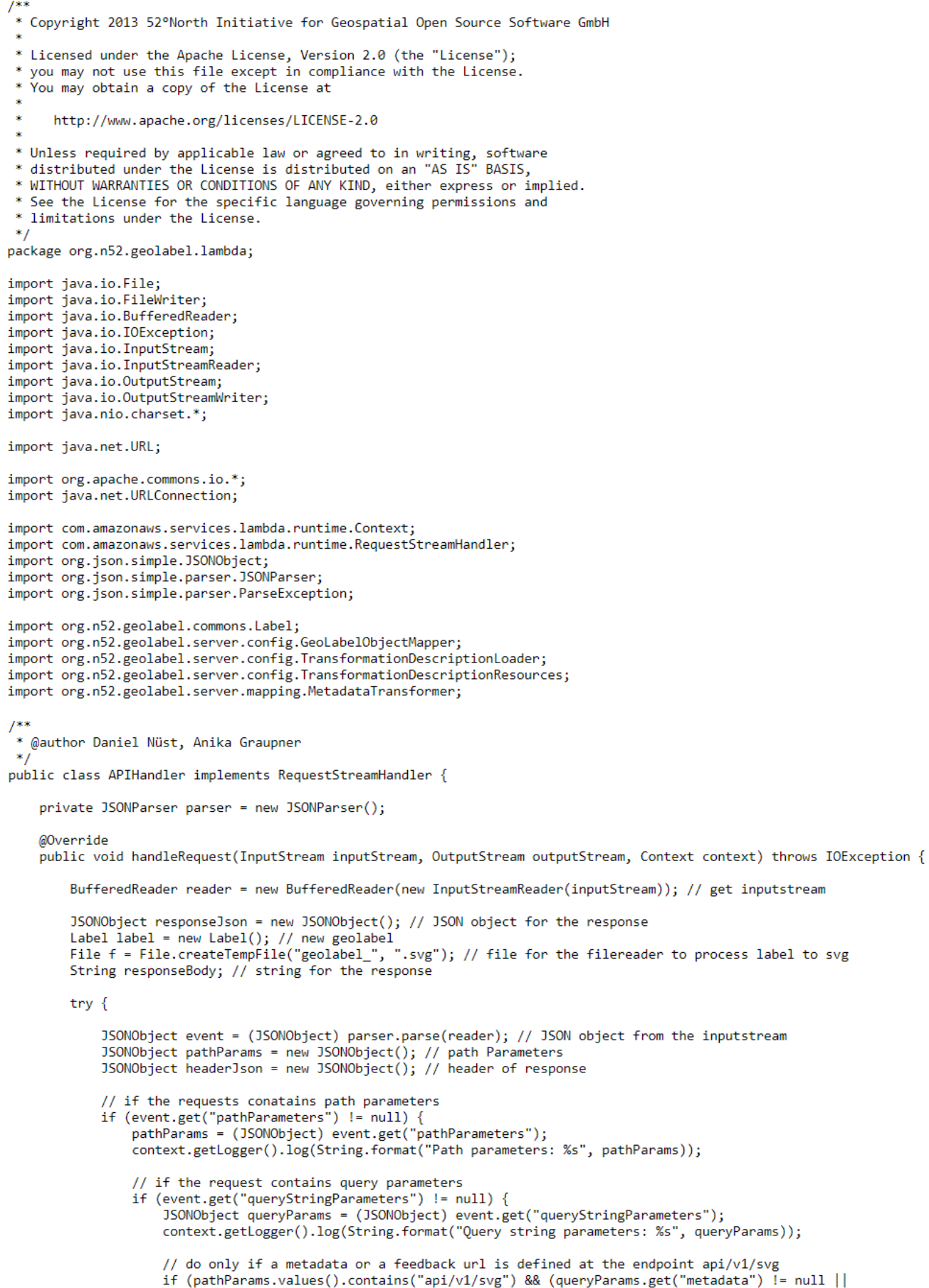

// transformation descriptions

MetadataTransformer transformer;
TransformationDescriptionResources res $=$ new

TransformationDescriptionResources("http://geoviqua.github. io/geolabel/mappings/transformer. json=/transformations/transformer.json, htt p://geoviqua.github.io/geolabel/mappings/transformerSML101.json=/transformations/transformerSML101.json, http://geoviqua.github.io/geol p://geoviqua.github.io/geolabel/mappings/transformerSML101.json=/transformations/transformerSML101.json, http://geoviqua.github.io/geol
abel/mappings/transformerSOS20.json=/transformations/transformerSOS20.json, http://geoviqua.github.io/geolabel/mappings/transformerSML2 $\theta$.json=/transformations/transformerSML20.json, http://geoviqua.github.io/geolabel/mappings/transformerSSNO.json=/transformations/transf o.json=/transformatid
ormerSSNO.json");

transformer $=$ new MetadataTransformer(new TransformationDescriptionLoader(res, 


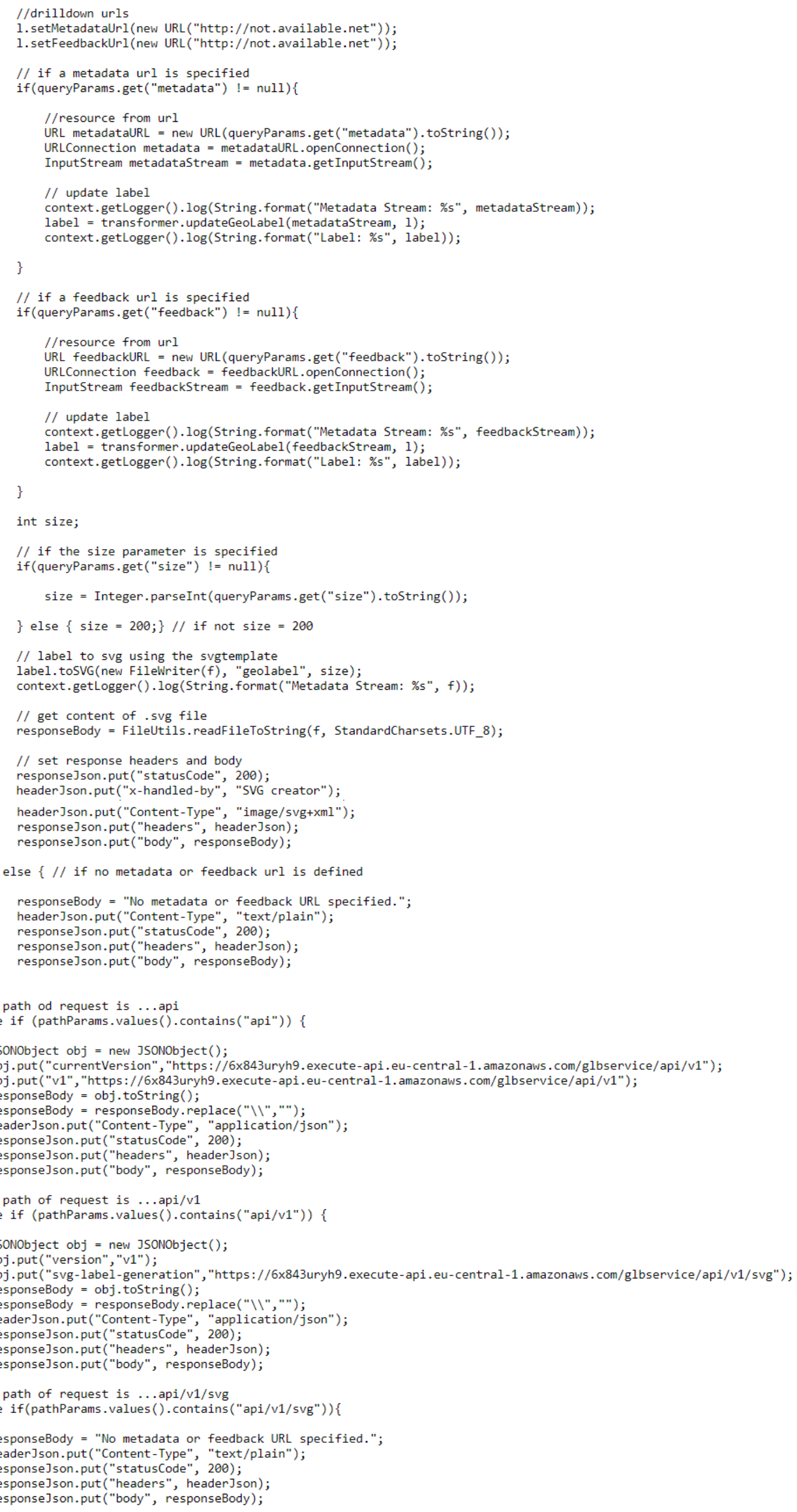


// if something went wrong by handling the request

\} catch (ParseException pex)

response]son, put ("statusCode", 400); responseJson.put("exception", pex.toString());

// OutputStream for Response

OutputStreamWriter writer $=$ new OutputStreamWriter(outputStream, "UTF-8"); writer.write(response]son.toString ()); writer.close ():

Anmerkung: Die Datei ist verfügbar unter https://github.com/anikagraupner/GEO-labeljava/blob/master/lambda/src/main/java/org/n52/geolabel/lambda/APIHandler.java. 


\section{Anhang 10: Fehlermeldungen JMeter bei 1000 gleichzeitig gesendeten Anfragen}

javax.net.ssl.sSLHandshakeException: Remote host terminated the handshake

at java.base/sun.security.ssl.SSLSocketImpl.handleEOF (SSLSocketImpl.java:132

at Java.base/sun.securlty.ssl.SsLsocketImpl.decode (SSLSocketImpl.java:1160)

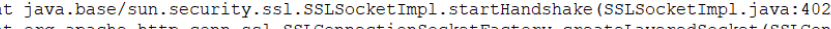

(SSLConnectionsocketFactory.java: 436

at org apache, http conn. ssl. SSLConnectionSocketFactory connectSocket (SSLConnectionSocketFactory, java: 384)

(S)

at org.apache.http.impl.conn. DefaultHttpclientConnectionoperator.connect (DefaultHttpclientConnectionoperator.java: 142)

at org. apache.jmeter.protocol.http. sampler. HTTPHC4 Impl s JMeterDefaultHttpClientConnectionoperator.connect (HTTPHC4 Impl.java: 326 )

at org.apache.http.impl.conn. PoolingHttpclientConnectionManager. connect (PoolingHttpclientConnectionManager.java: 374 )

the

to

at org.apache.http.impl.execchain.RetryExec.execute (RetryExec.java: 89 )

t org.apache.http.impl.execchain.RedirectExec.execute (RedirectExec.java:110)

at org.apache.http.impl.client. InternalHttpClient. doExecute (InternalHttpclient. java:185)

t. rapache.http.impl.client. CloseableHttpclient. execute (CloseableHttpclient. java: 83)

at org.apache.jmeter.protocol. http. sampler. HTTPHC 4 Impl. executeRequest (HTTPHC4 Impl.

at org.apache.jmeter.protocol.http. sampler. HTTPSamplerProxy. sample (HTTPSamplerProxy. java: 67)

at org.apache. jmeter.protocol.http.sampler. HTTPSamplerBase. sample (HTTPSamplerBase. java: 1282)

at org.apache.jmeter.protocol.http. sampler. HTTPSamplerBase. sample (HTTPSamplerBase. java:1271)

at org.apache.jmeter.threads. JMeterThread.doSampling (JMeterThread. java:627)

t org.apache. juet.

(j)

at java.base/java.lang. Thread.run(Thread.java:834)

Caused by: java.io.EOFException: SSL peer shut down incorrectly

at java.base/sun. security. ssl.SSLSocketInputRecord, decode (SSLSocketInputRecord.java:167)

at java.base/sun.security.ssl.sSLTransport.decode (SSLTransport.java:108)

at java.base/sun.security.ssl.sSLSocketImpl.decode (SSLSocketImpl.java:1152

... 25 more

avax.net.ssl.SSLException: Connection reset

sun. security.ssl.Alert.createSSLException (Alert. java:127) jo

at java.base/sun.security.ssl.TransportContext.fatal (TransportContext.java:321)

at java.base/sun.security.ssl.Transportcontext. fatal (TransportContext.java:264)

at java.base/sun.security.ssl.Transportcontext.fatal (TransportContext.java:259)

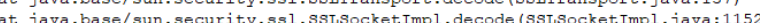

at java.base/sun.security.ssl.sSISocketImpl.readHandshakeRecord (SSISocketImpl.java: 1063)

at java.base/sun.security.ss1.sSLSocketImpl.startHandshake (SSLSocketImpl.java: 402)

at org.apache.http.conn.ssl.SSLConnectionSocketFactory.createLayeredSocket (SSIConnectionSocketFactory.java: 436)

at org.apache, http.conn. ssl. SSLConnectionsocketFactory, connectSocket (SSLConnectionSocketFactory java: 384)

at org.apache.jmeter.protocol.http, sampler.hc. IazyLayeredConnectionsocketFactory, connectSocket (IazyLayeredConnectionsocketFactory. java: 92 )

at org.apache.http.impl.conn. DefaulthttpclientConnectionoperator.connect (DefaulthttpclientConnectionoperator.java: 142)

at org.apache.jmeter.protocol.http. sampler. HTTPHC4 Impl\$ JMeterDefaultHttpClientConnectionoperator.connect (HTTPHC4 Impl.java: 326 )

作

at org.apache.http.impl.execchain.Mainclientexec.establishRoute (MainclientExec.java: 393 )

at orgat

torg.

torg apache http.implexecchain Redirectexec, execute(RedirectExec, java:110)

at org.apache.http.impl.client.InternalHttpclient.doExecute (InternalHttpclient.java:185)

at org.apache.http.impl.client.closeableHttpclient. execute (CloseableHttpClient. java: 83)

at org. apache. jmeter. protocol.http. sampler. HTTPHC4 Impl. executeRequest (HTTPHC4 Impl. java

at org.apache.jmeter.protocol.http. sampler. HTT PHC4 Impl. sample (HTTPHC4 Impl. java: 561)

at org.apache.jmeter.protocol.http. sampler. HTTPSamplerProxy. sample (HTTPSamplerProxy . java: 67 )

at org.apache.jmeter.protocol.http. sampler. HTPSamplerBase. sample (HTTPSamplerBase.java:1282)

at org.apache.jmeter.threads. JMeterThread.doSampling (MMeterThread. java:627)

at org.apache.jmeter. threads. JMeterThread. executesamplePackage (JMeterThread.java: 551 )

at org.apache.jmeter.threads. JMeterThread.processsampler (JMeterThread. java: 490 )

at org apache,jmeter, threads. JMeterThread, run (JMeterThread, java:257)

at java base/java. lang. Thread, run (Thread, java:834)

Suppressed: java.net.Socketexception: Connection reset by peer: socket write error

at java.base/java.net. SocketoutputStream. socketWriteo (Native Method)

at Java.base/Java.net. SocketoutputStream. socketWrite (SocketoutputStream.ja

at java.base/java.net.SocketoutputStream.write(SocketoutputStream.java:150)

at java.base/sun.security.ssl.SSLSocketOutputRecord.encodeAlert (SSLSocketoutputRecord. java: 81)

java.base 29 more

Caused by: java.net. SocketException: Connection reset

at java.base/java.net. Socket Inputstream.read (Socket Inputstream. java: 186)

at java.base/sun.security.ssl.SSLSocket InputRecord.read (SSLSocket InputRecord.java : 448)

(SSLSocketInputRecord. java:165)

at java.base/sun.security.ssl.sSLTransport.decode (SSLTransport.java:108)

.. 26 more 


\section{Plagiatserklärung}

Hiermit versichere ich, dass die vorliegende Arbeit über „Ein Metadatenlabel für das semantische Sensorweb" selbstständig verfasst worden ist, dass keine anderen Quellen und Hilfsmittel als die angegebenen benutzt worden sind und dass die Stellen der Arbeit, die anderen Werken - auch elektronischen Medien - dem Wortlaut oder Sinn nach entnommenen wurden, auf jeden Fall unter Angabe der Quelle als Entlehnung kenntlich gemacht worden sind.

(Datum, Unterschrift)

Ich erkläre mich mit einem Abgleich der Arbeit mit anderen Texten zwecks Auffindung von Übereinstimmungen sowie mit einer zu diesem Zweck vorzunehmenden Speicherung der Arbeit in eine Datenbank einverstanden.

(Datum, Unterschrift) 